\title{
Flatland Nanophotonics: A Study of Quantum-Confined Excitons in 2D Materials
}

\author{
by \\ Chitraleema Chakraborty \\ Submitted in Partial Fulfillment of the \\ Requirements for the Degree \\ Doctor of Philosophy \\ Supervised by \\ Professor Nick Vamivakas \\ Materials Science Program \\ Arts, Sciences and Engineering \\ Hajim School of Engineering and Applied Sciences \\ University of Rochester \\ Rochester, New York
}

2018 
For Bapi and Mummy 


\section{Table of Contents}

Biographical Sketch viii

Acknowledgments $\quad$ xi

$\begin{array}{ll}\text { Abstract } & \text { xvi }\end{array}$

Contributors and Funding Sources xviii

List of Figures $\quad$ Xx

1 Flatland materials: An overview 1

1.1 Introduction . . . . . . . . . . . . . . . . . . . 1

1.2 Graphene ............................. 3

1.2.1 Mechanical exfoliation . . . . . . . . . . . . 5

1.2 .2 Identification . . . . . . . . . . . . . . . 6

1.3 Hexagonal Boron nitride . . . . . . . . . . . . . . . 8

1.4 Transition metal dichalcogenides . . . . . . . . . . . . . . . . . 10

1.4.1 Crystal Structure . . . . . . . . . . . . . . . . 11

1.4.2 Band structure . . . . . . . . . . . . . . . . 12 
1.4.3 Preparation and identification ............ 14

Raman spectra of monolayer TMDCs . . . . . . . . 15

1.5 Van der Waals heterostructures . . . . . . . . . . . . 16

1.5.1 Assembling the layers . . . . . . . . . . . . . . 17

1.6 Thesis outline . . . . . . . . . . . . . . . . . . . . . . . . . . 18

2 Flatland excitons $\quad 21$

2.1 Excitons in $2 \mathrm{D} \ldots \ldots \ldots . \ldots . \ldots 21$

2.1.1 Photoluminescence ................ . . 23

Material and layer dependence . . . . . . . . . . . 23

Temperature dependence . . . . . . . . . . . . 25

2.1.2 Reflectance ................. . . 26

2.2 Charged excitons ..................... 27

2.3 Concept of Valleytronics . . . . . . . . . . . . . . . 28

2.3.1 Valley excitons: Polarization and Coherence . . . . . . . . 31

2.3.2 Valley Zeeman effect . . . . . . . . . . . . . . 32

2.4 0D Excitons in the $2 \mathrm{D} \ldots \ldots . \ldots . \ldots 34$

2.5 Interlayer excitons . . . . . . . . . . . . . . . 35

2.6 Summary ....................... 36

3 Quantum light emission from 2d semiconductors $\quad 37$

3.1 Introduction . . . . . . . . . . . . . . . . . . . 37

3.2 Sample preparation . . . . . . . . . . . . . . . . . 39

3.3 Setup........................... 40 
3.3.1 Intensity autocorrelation setup . . . . . . . . . . . 42

3.3.2 Polarization resolved setup . . . . . . . . . . . . 42

3.4 Optical identification: Micro-Photoluminescence spectroscopy . . 44

3.4.1 Power dependence . . . . . . . . . . . . . . 46

3.4.2 Temperature dependence . . . . . . . . . . . . . 48

3.4.3 Spectral Wandering ............... . 49

3.5 Photon statistics ...................... 50

3.6 Dipole orientation . . . . . . . . . . . . . . . 53

3.7 Magnetic field dependence . . . . . . . . . . . . . . . 54

3.7.1 Fine structure splitting . . . . . . . . . . . 54

3.7.2 Zeeman effect in $\mathrm{WSe}_{2} \mathrm{QD} \ldots \ldots . \ldots . \ldots 55$

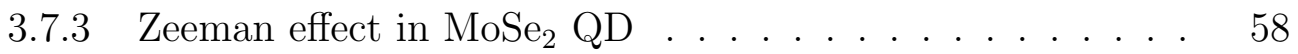

3.8 Gate voltage dependent emission . . . . . . . . . . . . 60

3.9 Strain dependent emission . . . . . . . . . . . . . . 61

3.10 Conclusion . . . . . . . . . . . . . . . . . . 62

4 Quantum confined Stark effect of single photon emitters in WSe 64

4.1 Introduction . . . . . . . . . . . . . . . 65

4.2 Heterostructure assembly and characterization . . . . . . . . 66

4.2.1 Device fabrication . . . . . . . . . . . 67

4.2.2 Device characterization . . . . . . . . . . 67

4.2.3 Confirmation of quantum light emission . . . . . . . 69

4.3 Demonstration of Stark Effect . . . . . . . . . . . . . 70

4.3.1 Photoluminescence vs vertical electric field . . . . . . . . 70 
4.3.2 Spectral wandering vs Stark shift . . . . . . . . . . 72

4.3.3 Fit to Stark shift equation . . . . . . . . . 73

4.3 .4 Statistics . . . . . . . . . . . . 76

4.4 Influence of the magnetic field . . . . . . . . . . . . . . . 78

4.5 Summary ......................... 81

5 Manipulation of the fine structure splitting of the quantum dot $\begin{array}{ll}\text { exciton } & 82\end{array}$

5.1 Modulation of the fine structure splitting . . . . . . . . . . 83

5.2 Circular polarization vs FSS . . . . . . . . . . . . . 85

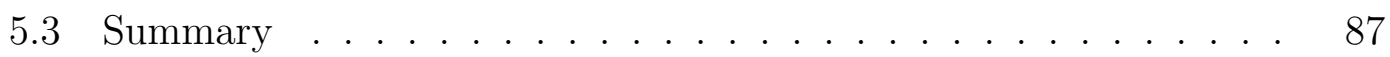

$63 \mathrm{D}$ localized exciton complexes in a charge tunable van der Waals $\begin{array}{lr}\text { heterostructure } & 88\end{array}$

6.1 Introduction . . . . . . . . . . . . . . . . 89

6.2 Device architecture . . . . . . . . . . . . . . . . 90

6.3 Identification of neutral exciton, trion and biexciton . . . . . . . 91

6.4 Power dependence of the voltage plateaus . . . . . . . . . . . . 93

6.5 Polarization dependence . . . . . . . . . . . . . . . 95

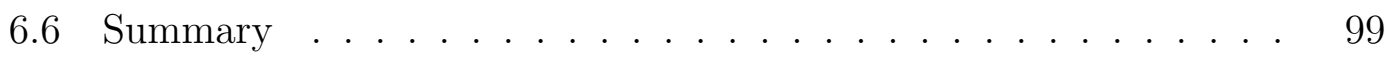

7 Integration of 2D materials with plasmonic and photonic devices 100

7.1 Optical antenna enhanced graphene photodetector . . . . . . . . . 100

7.1.1 Introduction . . . . . . . . . . . . . . . 101

7.1.2 Device structure. . . . . . . . . . . . . 102 
7.1.3 Simulation and measurements . . . . . . . . . . . 103

7.1 .4 Summary . . . . . . . . . . . . . . 110

7.2 Direct on-chip plasmon detection with monolayer $\mathrm{MoS}_{2}$. . . . . 111

7.2 .1 Introduction . . . . . . . . . . . . . . 111

7.2.2 Device geometry and fabrication . . . . . . . . . . 112

7.2.3 Scanning photocurrent microscopy . . . . . . . . . . . . 114

7.2.4 Figure of merits of the detector . . . . . . . . . . . . 116

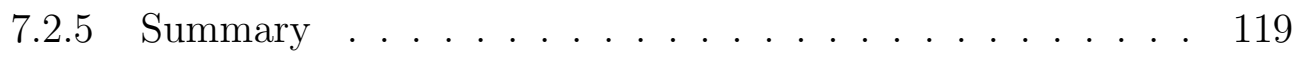

7.3 Exciton polariton in 2D TMDC . . . . . . . . . . . . 120

7.3 .1 Introduction . . . . . . . . . . . . . . . 120

7.3.2 Device architecture . . . . . . . . . . . . . 121

7.3.3 Optical characterization . . . . . . . . . . . 121

7.3.4 Imaging the dispersion . . . . . . . . . . . . . . 123

Optical setup . . . . . . . . . . . . . . . 123

Calibration ................... 124

7.3.5 The cavity coupled $\mathrm{MoSe}_{2} \ldots \ldots . \ldots . . \ldots 125$

7.3.6 Deviation from positive dispersion . . . . . . . . . . 126

7.3.7 Summary . . . . . . . . . . . . . . . . . . 128

8 Conclusion and perspective $\quad 129$

8.1 Overall summary . . . . . . . . . . . . . . . . . 129

8.2 Perspective . . . . . . . . . . . . . . . . . . . . 131

$\begin{array}{lr}\text { Bibliography } & 134\end{array}$ 


\section{Biographical Sketch}

The author was born and brought up in the city of Hooghly in West Bengal, India. She attended Jadavpur University in Kolkata from 2006 to 2009 and graduated with a Bachelor of Science degree with honors in Physics. Following that, she joined the master's program in Nanoscience and Nanotechnology at the University of Delhi, New Delhi, India in collaboration with Joseph Fourier University, Grenoble, France. She received a Master of Science degree in Nanophysics and Nanostructures from Joseph Fourier University in 2011 and a Master of Technology degree in Nanoscience and Nanotechnology from the University of Delhi in 2012. She also worked as a project student at the Tata Institute of Fundamental Research in Mumbai under the supervision of Professor Mandar M. Deshmukh. During the fall of 2012, she enrolled in the Materials Science doctoral program at the University of Rochester. In 2013, she joined the Quantum Nanophotonics group under the supervision of Professor Nick Vamivakas at The Institute of Optics where she explored the excitons in 2D materials by integrating them in nanophotonic and optoelectronic devices and probing them using optical spectroscopy. 


\section{List of Publications}

[1] C. Chakraborty, L. Qiu, K. Konthasinghe, A. Mukherjee, S. Dhara, and N. Vamivakas. 3D localized trions in monolayer $\mathrm{WSe}_{2}$ in a charge tunable van der Waals heterostructure. Nano Letters, 10.1021/acs.nanolett.7b05409, (2018).

[2] S. Dhara*, C. Chakraborty*, L. Qiu, K. M. Goodfellow, T. Oloughlin, G. W. Wicks, and S. Bhattacharjee, and N. Vamivakas. Anomalous dispersion of microcavity trion-polaritons. Nature Physics, 14(2):130-133, (2018)

[3] C. Chakraborty, K. M. Goodfellow, S. Dhara, A. Yoshimura, V. Meunier, and N. Vamivakas. Quantum-confined Stark effect of individual defects in a van der Waals heterostructure. Nano Letters, 17(4):2253-2258, (2017).

[4] C. Chakraborty, K. M. Goodfellow, and N. Vamivakas. Localized emission from defects in $\mathrm{MoSe}_{2}$ layers. Optical Materials Express, 6(6):2081-2087, (2016).

[5] K. M. Goodfellow, C. Chakraborty, P. Waduge, K. Sowers, M. Wanunu, T. Krauss, K. Driscoll, and N. Vamivakas. Distance-dependent energy transfer between CdSe/CdS quantum dots and a two-dimensional semiconductor. Applied Physics Letters, 108(2):021101, (2016).

[6] C. Chakraborty, L. Kinnischtzke, K. M. Goodfellow, R. Beams, and N. Vamivakas. Voltage-controlled quantum light from an atomically thin semiconductor. Nature Nanotechnology, 10(6):507-511, (2015).

[7] L. Kinnischtzke, K. M. Goodfellow, C. Chakraborty, Y. Lai, A. Badolato, and N. Vamivakas. Graphene mediated Stark shifting of quantum dot energy levels. Applied Physics Letters, 108(21):211905, (2015). 
[8] K. M. Goodfellow*, C. Chakraborty*, R. Beams, L. Novotny, and N. Vamivakas. Direct On-Chip Optical Plasmon Detection with an Atomically Thin Semiconductor. Nano Letters, 15(8):5477-5481, (2015).

[9] C. Chakraborty, R. Beams, K. M. Goodfellow, G. W. Wicks, L. Novotny, and N. Vamivakas. Optical antenna enhanced graphene photodetector. Applied Physics Letters, 105(24):241114, (2014).

[10] K. M. Goodfellow, R. Beams, C. Chakraborty, L. Novotny, and N. Vamivakas. Integrated nanophotonics based on nanowire plasmons and atomically thin material. Optica, 1(3):149, (2014).

[11] P. Parikh*, C. Chakraborty*, T. S. Abhilash, S. Sengupta, C. Cheng, J. Wu, and M. M. Deshmukh. Dynamically Tracking the Strain Across the Metal-Insulator Transition in $\mathrm{VO}_{2}$ Measured Using Electromechanical Resonators. Nano Letters, 13(10):4685-4689, (2013). 


\section{Acknowledgments}

As the most formative, blissful, exhaustive yet charming years of my life as a graduate student are nearing its end, I would like to extend my appreciation to everyone who helped me scientifically and emotionally throughout this journey.

I would like to begin by thanking my advisor Dr. Nick Vamivakas for believing in me and giving me a home in his lab for the last five years. His breadth of knowledge, the way he simplifies complex problems and his calm attitude has always been a model for me. His continuous guidance and feedback has been priceless and have helped me overcome many problems and frustrations. I really appreciate that he has always been very kind and approachable and kept his sense of humor at all times even when I lost mine. I had very little knowledge about optical setups when I joined the group. There were times when I took unreasonable long time to align even simple optics, yet, he never lost his patience. He always encouraged me to explore and follow my own ideas and remained motivating, reminding that mistakes are part of the learning curve. I am also indebted to him for sending me to multiple domestic and international conferences which helped me build a network and decide my next career move. Being the first wave of his students, I also got the luxury of getting hands on training directly from him in the lab. Working with him has been a really wonderful experience. Being his student will always be a pride for me. 
I thank Professor Gary Wicks and Professor Todd Krauss for their willingness to be on my qualifiers and dissertation committee. I am extremely grateful to Professor Nicholas Bigelow for agreeing to serve as the chair. I express my heartfelt gratitude to Professor Lukas Novotny from ETH Zurich for being so generous and supporting my graduate research initially so I could have a smooth start at Rochester. I feel fortunate that I had the opportunity to collaborate with him and discuss my research. I also thank Professor Roman Sobolewski from the Laboratory of Laser Energetics for letting me spend some time in his lab during my first year in Rochester.

Over the years, I have been very lucky for having the opportunity to work with some of the finest graduate students and postdocs. I am glad I had Dr. Ryan Beams, Dr. Sajal Dhara and Dr. Kumarasiri Konthasinghe, three incredibly smart post docs who were always available and eager to share their ideas and advice. Ryan was my first mentor in Rochester. I thank him for going through the tedious process of training me on the Near-Field microscope. I owe him all my alignment skills in optics. I also thank him for introducing me to espresso. It was a pleasure to share the espresso passion with Ryan, Steve, Barbara, Per, Sajal and Kumar. Thank you to Sajal for all his guidance and suggestions. I have known him for a long time, since he was a graduate student at TIFR, India. I am glad that we crossed paths again and became such close friends. I also thank his family: Deblina, Simul, Kaku and Kakima for offering me their love, friendship, incredibly great Bengali food and making Rochester feel like home. I have really enjoyed working with Kumar in the lab and discussing about Quantum Optics. I thank Kenny for teaching me the right way to exfoliate 2D materials and showing me how to stack them. I am extremely thankful to two of my smart little elves, Arunabh and Claude, for working with me during the final stage of my PhD. It 
was a really enjoyable teaching-learning experience. I am sure they will be great successors to the $2 \mathrm{D}$ material projects. I thank all the past and present members of the lab: Laura, Kenny, Tanya, Levi, Ryan, Rohit, Alex, Sajal, Arunabh, Claude, Robby, Justin, Danika, Wadood, Fei, Jeremy, Xiao Feng and Kumar. All of them have been fabulous colleagues and friends and I will really miss them!

A big thank you to Barbara Schirmer, Lynda McGarry, Gina Eagen, Noelene Votens, Lori Russell, Per Adamson, Brian McIntyre, Jim Mitchell, Alex Mann, Laura Blumkin, Mike Culver and all other staff members of The Institute of Optics, Materials Science Program and Department of Physics and Astronomy for their generous help and support over the years. I would like to extend my appreciation to Alan Bleier, Aaron Windsor, Jerry Drumheller and all staff members at the Cornell Nanoscale Facility in Ithaca for teaching me the nano-fabrication skills that I inculcated during these years.

I will forever remain thankful to my master's thesis advisor, Professor Mandar Deshmukh and his entire group at Tata Institute of Fundamental Research (TIFR), Mumbai, India. His enthusiasm and scientific curiosity was contagious. The first time I realized that I really enjoy doing research was after I joined his group. This strongly motivated me to pursue a PhD. I still think fondly of my time as a project student in his lab. I also thank all his group members, Shamashis, Sudipta, John, Abhilash, Vibor, Padmalekha, Sameer and Sajal for letting me participate in their projects which built my foundation of nano-electronics and nano-fabrication and led to a great start in Rochester. I believe the base of this thesis actually began there and this entire experience had a strong influence in modifying my general perspective towards research. I would also like to thank Achint who was a project student at TIFR with me and later moved to ETH and continued to give valuable advice on 2D materials based devices, transfer pro- 
cesses, etc. Also, my sincere and heart-felt gratitude to Professor S. Annapoorni, the graduate coordinator of the Nanoscience Masters Program in Delhi University for designing such a wonderful course, providing the amazing opportunity to join the Nanophysics Masters program in Grenoble, France and the valuable suggestions during the $\mathrm{PhD}$ application process.

I also thank all my friends outside the lab for all the non-PhD fun. I would like to start by thanking my girl gang: Deblina, Madhubanti, Sanghita, Preeti and Prabha. I thank them for all the moments we spent eating, chatting, shopping, celebrating, and the list goes on. I will always cherish these memories. I also thank Rupam, Ankani, Saikat, Shruthi, Sayak, Tanya, Rahul, Somjit and Sutirtho for sharing endless meals, wonderful trips and funny, yet insightful conversations. Without their warmth, it would have been impossible to survive the Rochester cold.

I thank Arijit, my best friend and husband. I am really grateful that he persuaded me to apply to the PhD program in Rochester. He has been immensely helpful during this entire process, starting from guiding me for the GRE tests, helping me get through with the graduate application process, finding a $\mathrm{PhD}$ supervisor, to thesis writing. Not to mention the comfort he created by managing all the chores whenever I went through any crisis. He was there with me during this entire time and I cannot thank him enough for that.

I am extremely lucky to have two bratty, yet affectionate rabbits, Chotu and Koko. They added a lot of happiness to this whole experience by always greeting me with enthusiasm no matter what, spending hours entertaining me with their interesting ways of expressing themselves and literally being there with me throughout the time as I typed up this dissertation. They are a really wonderful company. 
Finally, I want to thank my family back in India, without whose endless love and support, none of this would have been possible. I want to thank my mother for all the sacrifices she made for making sure that I consistently had access to the best education. I thank my father for encouraging me to pursue the career I wanted and always inspiring me to work harder since I was a little girl. He has been and will always remain my best role model. It has been a long journey and I could not have done any of it without their belief in me. 


\section{Abstract}

Semiconducting two-dimensional materials have gained increasing scientific interest within the last decade. Their electronic band gap in the visible range of the electromagnetic spectra, intriguing properties associated with spin and valley pseudospin of carriers and strongly bound excitons make them an excellent platform for both fundamental research, and technology tailored towards applications in nanophotonics and optoelectronics. Recently, 3D localized excitons in 2D materials have emerged as a novel source of single photon emitters, thus, unlocking the potential of these flatland materials in quantum optics and quantum information technology. The discovery of these localized excitons and the advances made in the study of quantum emitters in $2 \mathrm{D}$ materials are the major contributions of this thesis.

In this thesis, we study quantum-confined excitons in monolayer transition metal dichalcogenides (TMDCs), a semiconducting class of 2D material. The effects of 3D confinement of excitons in the host monolayer TMDCs are investigated by optical spectroscopy. Low-temperature photoluminescence emission from the localized excitons exhibits narrow linewidths ranging from $100 \mu \mathrm{eV}-500 \mu \mathrm{eV}$ with peak energies that are red-shifted from the delocalized excitons. Photon antibunching in intensity autocorrelation measurement confirms their single-photon nature. Magneto-optical studies reveal an exciton g-factor of $\sim 10$. Next, electricfield tunable devices based on van der Waals heterostructure are built around these 
localized emitters hosted by monolayer TMDC to study the quantum-confined Stark effect and demonstrate the electrical modulation of their photophysical properties such as emission energy, intensity, linewidth and fine structure splitting. We also investigate fully localized trions embedded in a charge-tunable van der Waals heterostructure. In such a device, direct electrostatic doping results in the formation of quantum confined trions with reduced electron-hole exchange interactions manifested by a reduction in the fine structure splitting and enhanced degree of circular polarization. This fosters the possibility of fabricating optically controlled spin-valley qubits with 2D materials.

Lastly, we present various integrated devices based on $2 \mathrm{D}$ materials that are coupled with nanostructures such as metallic nano-antenna, nanowire-based waveguide and planar optical cavity based on distributed Bragg reflectors. These devices not only serve as a platform for solid-state quantum optics research but also provide building blocks for future nanophotonic and optoelectronic circuits. 


\section{Contributors and Funding Sources}

This work was supervised by a dissertation committee consisting of Professor Nick Vamivakas (advisor) and Professor Gary Wicks from The Institute of Optics,

and Professor Todd Krauss from the Department of Chemistry, University of Rochester.

Parts of Chapter 3 were previously published in Nature Nanotechnology in 2015 and Optical Materials Express in 2016. The work in chapter 4 was published in Nano Letters in 2017. The work in chapter 6 was published in Nano Letters in 2018. Sections 7.1, 7.2 and 7.3 were published in Applied physics Letters (2014), Nano Letters (2015) and Nature Physics (2018) respectively. All of the above publications are listed in the biographical sketch. The author shares equal contribution with Dr. Kenneth Goodfellow and Dr. Sajal Dhara in the research conducted for section 7.2 and and section 7.3 respectively.

This material is based upon work supported by the Institute of Optics, National Science Foundation (DMR-1309734, NSF-EFMA-1542707 and NSF-DMR1553788, DGE-0966089, DMR-1309734), the Air Force Office of Scientific Research (AFOSR-FA9550-16-1-002), U.S. Department of Energy (Grant No. DE-FG0205ER46207), the Army Research Office (W911NF1110378) and the Swiss National Science Foundation (200021-149433). The sample used in section 7.1 and 7.2 is 
partly fabricated at the Cornell NanoScale Facility, a member of the National Nanotechnology Infrastructure Network, which is supported by the National Science Foundation (Grant No. ECCS-0335765).

\section{Copyright Permissions:}

- Reprinted with permission from Nature Nanotech. 10, 6, 507-511 (2015).

- Reprinted (adapted) with permission from (Nano Letters, 17, 4, 2253-2258, 2017). Copyright (2017) American Chemical Society.

- Reprinted (adapted) with permission from (Nano Letters, 10.1021/acs.nanolett.7b05409, 2018). Copyright (2018) American Chemical Society.

- Reprinted from Appl. Phys. Lett. 105, 24, 241114 (2014), with the permission of AIP Publishing.

- Reprinted (adapted) with permission from (Nano Letters, 15, 8, 5477-5481, 2015). Copyright (2015) American Chemical Society.

- Reprinted with permission from Nature Physics. 14, 2, 130-133 (2018). 


\section{List of Figures}

1.1 Graphene lattice . . . . . . . . . . . . . . . . . 4

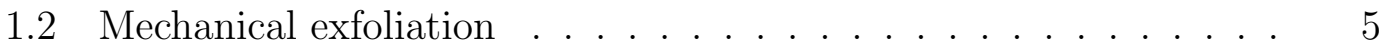

1.3 An optical micrograph of an exfoliated graphene . . . . . . 6

1.4 Raman spectra of monolayer graphene and few-layer graphene. . . 7

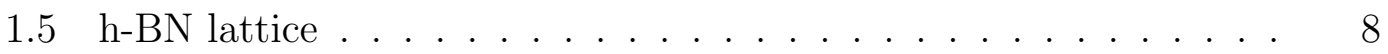

1.6 Monolayer TMDC lattice . . . . . . . . . . . . . . . . . . . 11

1.7 TMDC band structure $\ldots \ldots \ldots \ldots \ldots \ldots$

1.8 Raman spectra of $\mathbf{a} \cdot \mathrm{MoS}_{2}$ b. $\mathrm{MoSe}_{2}$ and $\mathbf{c} \cdot \mathrm{WSe}_{2} \ldots \ldots \ldots$

1.9 Dry transfer technique . . . . . . . . . . . . . . . 17

2.1 Photoluminescence . . . . . . . . . . . . . . . . . . 23

2.2 Layer dependent PL . . . . . . . . . . . . . . . . . . . . 24

2.3 Temperature dependent PL spectra of $\mathbf{a} . \mathrm{MoS}_{2}, \mathbf{b} . \mathrm{WSe}_{2}$ and $\mathbf{c}$.

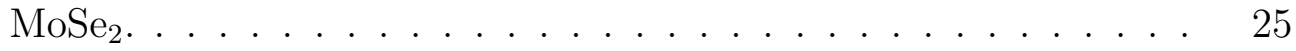

2.4 Reflectance . . . . . . . . . . . . . . . . 26

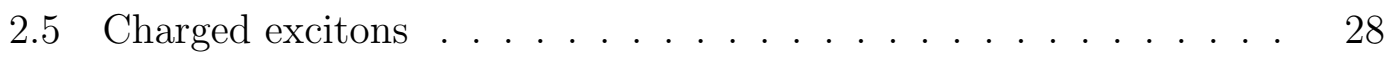

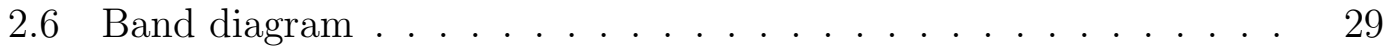


2.7 Polarization resolved $\mathrm{PL} \ldots \ldots . \ldots 32$

2.8 Valley Zeeman effect ................... 33

3.1 Schematic of the device . . . . . . . . . . . . . 39

3.2 Low temperature magneto-optical setup . . . . . . . . . . . . 41

3.3 Polarization resolved setup . . . . . . . . . . . . . . . . 43

3.4 PL identification . . . . . . . . . . . . . . . . 44

3.5 Hyperspectral data .................. 46

3.6 Power dependence. . . . . . . . . . . . . . . . . . . . . . 47

3.7 Temperature Dependence . . . . . . . . . . . . . . . . 48

3.8 Spectral wandering ................... 50

3.9 Photon antibunching .................... 51

3.10 Dipole orientation: . . . . . . . . . . . . . . . . 53

3.11 Fine structure splitting: . . . . . . . . . . . . . . . 55

3.12 Magneto-optical studies of the localized emitters in $\mathrm{WSe}_{2}$. . . . 56

3.13 Polarization vs B field: . . . . . . . . . . . . . . . 57

3.14 Magneto-optical studies of the localized emitters in $\mathrm{MoSe}_{2}$. . . . 59

3.15 Gate voltage dependence . . . . . . . . . . . . . . 60

3.16 Strain dependent emission . . . . . . . . . . . . . . 61

4.1 Device assembly and characterization . . . . . . . . . . 68

4.2 Second order intensity autocorrelation measurement . . . . . . 69

4.3 PL vs voltage . . . . . . . . . . . . . . . . . 71

4.4 Spectral diffusion vs Stark shift . . . . . . . . . . . . . . 72 


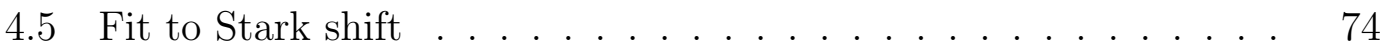

4.6 Variety of Stark shift . . . . . . . . . . . . . 75

4.7 Statistics of different varieties of Stark shift $\ldots \ldots \ldots \ldots$

4.8 Trend of the fit parameters . . . . . . . . . . . . 77

4.9 Statistical analysis $\ldots \ldots \ldots \ldots \ldots \ldots$

4.10 Magneto-optical studies _ . . . . . . . . . . . . . . . . . 79

4.11 Stark effect vs B field . . . . . . . . . . . . . . . . . . 80

5.1 Modulation of FSS . . . . . . . . . . . . . . . . 83

5.2 Analysis of the doublets . . . . . . . . . . . . 84

5.3 Circular polarization $\ldots \ldots \ldots \ldots \ldots$

6.1 Device architecture . . . . . . . . . . . . . . . . 91

6.2 PL vs voltage $\ldots \ldots \ldots \ldots \ldots$

6.3 Photoluminescence analysis . . . . . . . . . . . . . . 94

6.4 Polarization resolved PL $\ldots \ldots \ldots \ldots$

6.5 Valley polarization vs electric field f . . . . . . . . . . 98

7.1 Device schematic and SEM micrograph . . . . . . . . . . . 104

7.2 Simulation and measurements . . . . . . . . . . . . 105

7.3 Polarization and photoresponse . . . . . . . . . . 107

7.4 Scanning photocurrent map . . . . . . . . . . . . . . . 112

7.5 Polarization dependent photocurrent . . . . . . . . . . 115

7.6 Responsivity and efficiency . . . . . . . . . . . . . . . 117

7.7 Wavelength dependent photocurrent . . . . . . . . . 118 
7.8 Schematic of the DBR based cavity hosting the monolayer $\mathrm{MoSe}_{2}$

flake at the center of the cavity. . . . . . . . . . . . . . . 121

7.9 Optical characterization of bare $\mathrm{MoSe}_{2} \ldots \ldots \ldots$. . . . . . 122

7.10 Bare cavity vs loaded cavity . . . . . . . . . . . . . . 123

7.11 Schematic of the setup used to image the dispersion. . . . . . . 123

7.12 Setup calibration . . . . . . . . . . . . . . . 125

7.13 Angle resolved PL . . . . . . . . . . . . . . . . 126

7.14 Experiment vs coupled three oscillator model: . . . . . . . . . 127 


\section{Chapter 1}

\section{Flatland materials: An}

\section{overview}

Let us begin our journey by revisiting some of the history and fundamental properties of flatland materials - materials that are two-dimensional. In this chapter we will introduce the different types of two dimensional (2D) materials that we have studied in this work. We will also discuss some of the techniques of preparation and identification of these materials. Finally, a brief overview of the thesis will be presented.

\section{$1.1 \quad$ Introduction}

One characteristic of a material that determines much of its physical properties is its dimensionality. A material with a given chemical composition in a three dimensional (3D) crystal lattice can exhibit significantly different properties from the same material arranged in a quasi one-dimensional (1D) [1], two-dimensional (2D) or even quasi zero-dimensional (0D) [2] structure. Although layered materials have been studied by the scientific community for more than a century and half [3], their true potential has only been noticed on thinning down to its physical 
limits. This was realized after the isolation of graphene by Novoselov and Geim in 2004 [4], resulting in the Nobel Prize in Physics in 2010. Graphene is arguably the most studied 2D material because of its intriguing mechanical, electronic, optical, chemical and biological applications. The recent progress in nanoelectronics using graphene has demonstrated potential towards further miniaturization beyond Moore's law, as well as a high-mobility alternative to conventional semiconductors [5] in the emerging fields of large-area, flexible, and low-cost electronics. Graphene is only the first candidate in the ever expanding category of what are called the $2 \mathrm{D}$ materials. There has been a surge in the discovery of $2 \mathrm{D}$ materials beyond graphene [6] triggered by the desire to find non-metallic - insulating and semiconducting complements to graphene. The candidates for novel $2 \mathrm{D}$ materials are any compound with a layered lattice structure. Hexagonal boron nitride (hBN), a layered insulating material was studied as it was predicted to open up a band gap in graphene [7]. The next class of 2D materials isolated after graphene were the transition metal dichalcogenides. The TMDCs have electronic properties spanning semiconductors $\left(\mathrm{MoS}_{2}, \mathrm{MoSe}_{2}, \mathrm{WS}_{2}, \mathrm{WSe} \mathrm{C}_{2}\right.$, etc), insulators $\left(\mathrm{HfS}_{2}\right)$, semimetals $\left(\mathrm{WTe}_{2}\right)$, metals $\left(\mathrm{NbS}_{2}, \mathrm{VSe}_{2}\right)$ and even superconductors $\left(\mathrm{NbSe}_{2}\right)$ [8]. Other 2D materials include graphene composites like reduced graphene oxides [9], buckled crystal analogues of graphene like phosphorene, silicene and germanene [10]. Additional interesting families of layered materials are the III-VIs (such as InSe and GaS), the V-VIs (such as $\mathrm{Bi}_{2} \mathrm{Te}_{3}$ and $\mathrm{Sb}_{2} \mathrm{Se}_{3}$ ), the metal trichalcogenides, and metal trihalides [11]. Although, most of the "beyond-graphene" materials are not atomically thin, they are termed $2 \mathrm{D}$ as they represent the thinnest crystalline solids that can be realized from the individual materials. Furthermore, heterostructures based on stacking different 2D materials (also known as van der Waals heterostructures) showed further interesting properties due to the possibil- 
ity to combine and tailor various properties of the constituent materials [12].

The $2 \mathrm{D}$ materials that are mostly studied in this work are the semiconducting transition metal dichalcogenides (TMDCs), like, tungsten diselenide $\left(\mathrm{WSe}_{2}\right)$ in chapters 3 - 6, molybdenum diselenide $\left(\mathrm{MoSe}_{2}\right)$ in parts of chapters 3 and 7, molybdenum disulphide $\left(\mathrm{MoS}_{2}\right)$ in chapter 7 . Integrated photodetectors based on graphene will be discussed in chapter 7. While not studied directly, other materials like few layer hexagonal boron nitride and graphene have been used to build van der Waal's heterostructure for many of the experiments based on nanooptoelectronics with 2D TMDCs. In this chapter, we will briefly introduce the different materials that we have studied in experiments described in the subsequent chapters of this thesis.

\subsection{Graphene}

Bulk graphene, i.e., graphite existed in nature almost since the first chapter of earth's history [13]. Extensive research into graphene has discussed a number of outstanding properties. It has large electrical and thermal conductivity, the highest natural mechanical strength, it is highly resistant to chemical attack and exhibits self-lubricating properties owing to its layered nature. Graphene is a monolayer of graphite. Carbon has four valence electrons. Each carbon atom in graphene has orbitals that are $s p^{2}$ hybridized and bonded to three other carbon atoms resulting in a tightly packed 2D honeycomb lattice (Fig. 1.1). The inter atom separation in graphene is about $1.42 \stackrel{\AA}{A}[14]$. The fourth of these valence electrons occupies an orbital perpendicular to the one-dimensional (1D) sheet creating delocalized $\pi$ bonding which allows for the creation of a two-dimensional electron gas (2DEG) with high mobility within the sheets. The delocalization of the $\pi$ - 


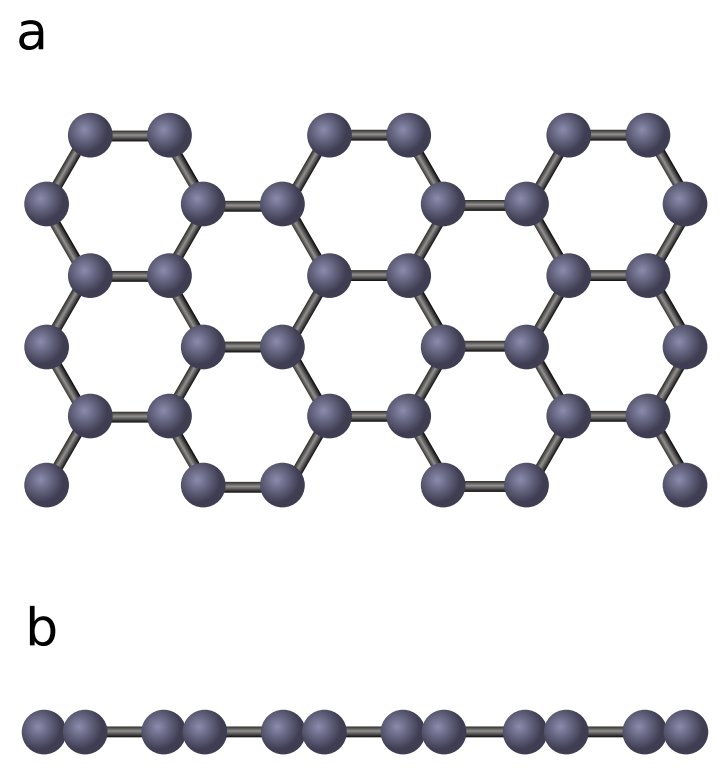

Figure 1.1: Graphene lattice: a. Top view of graphene b. Side view of graphene.

bonding electrons allows the graphene sheet to have a high carrier mobility, with a great potential for graphene-based electronic devices [15]. Due to the ambipolar nature of the field effect transistor with a graphene channel, charge carriers can be continuously tuned between electrons and holes in high concentrations [4]. However the lack of an intrinsic band gap limits its application in digital electronics. In spite of that, the unmatched properties of graphene as a transparent conductor continues making headways into various electronic applications outside of digital electronics. Transparent conductors are required for electrodes in various optoelectronic devices like touchscreens, solar cells and liquid crystal displays. For example, a graphene based touchscreen is expected to markedly reduce the production cost of devices that previously used Indium tin oxide (ITO), therefore allowing more affordable and flexible displays [16]. The ability of graphene to act as a thin and transparent contact is mostly exploited in this work. In addition, we have also demonstrated the integration of graphene with a nano-plasmonic antenna for photodetection in chapter 7 . 


\subsubsection{Mechanical exfoliation}
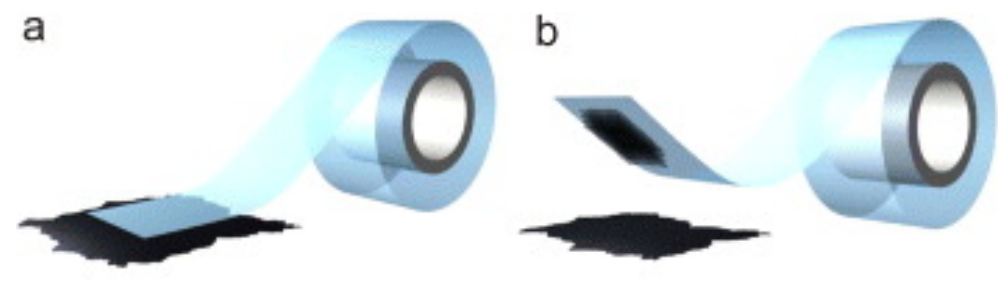

$\mathrm{C}$

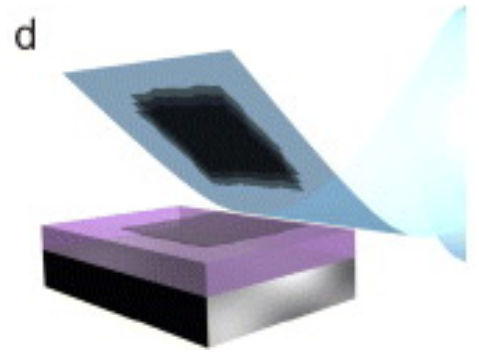

Figure 1.2: Mechanical exfoliation: a. An adhesive scotch tape is placed over the bulk material b. The tape is peeled off slowly and c. transferred to a substrate. d. The tape is peeled off from the substrate so that few layers remain on the substrate [17].

High quality single layers of 2D materials can be produced using micromechanical cleavage, more commonly known as the scotch tape technique. In 2004, this was first demonstrated by Novoselov et al. in their seminal article of isolating monolayer graphene from the parent graphite [4].

We use highly oriented pyrolitic graphite (HOPG) from SPI Supplies for all graphene related projects in this thesis. The mechanical exfoliation is detailed in Fig 1.2. Monolayer or few layer graphene is first exfoliated from bulk graphite using a sticky scotch tape (semiconducting dicing tape from Semicorp) and placed on a substrate (Fig 1.2a-c). The substrate used was either a highly doped silicon (N-type, phosphorus doped from MTI Corporation) with $300 \mathrm{~nm}$ thick silicon dioxide layer for facilitating optical identification of individual layers under a microscope [18] or a polymer based PDMS stamp (Gelpak, PF film) for constructing 
a heterostructure. The tape is then slowly peeled off from the substrate (Fig 1.2d). This method yields graphene monolayers up to few hundreds of microns in area. An exemplary graphene flake exfoliated on a $\mathrm{Si} / \mathrm{SiO}_{2}$ substrate is shown in Fig 1.3 .

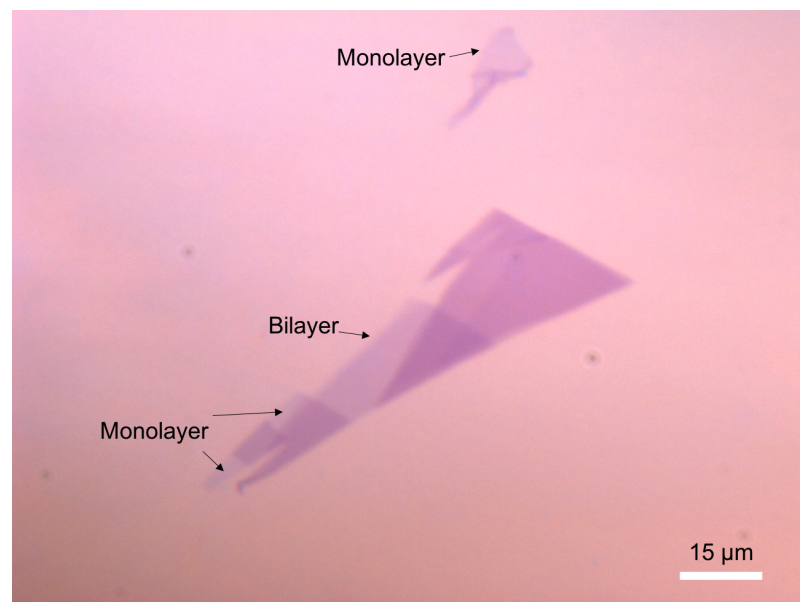

Figure 1.3: An optical micrograph of an exfoliated graphene flake on $\mathrm{Si} / \mathrm{SiO}_{2}$ substrate.

\subsubsection{Identification}

So far, one of the fastest ways to locate graphene or any other monolayer material is to exfoliate them on a $\mathrm{Si} / \mathrm{SiO}_{2}$ surface and then challenge the patience, eyesight and vigilance of a graduate student as he/she scans the optical microscope stage for micron sized flakes of monolayers present among multilayers of the exfoliated material. A contrast is observed even for the monolayer in Fig 1.3 because the thin film adds to an optical path that changes the interference color with respect to the empty wafer on the background of the flake [18]. Atomic force microscopy, transmission electron microscopy and Raman spectroscopy are some of the techniques employed to confirm the thickness and related properties of the graphene 


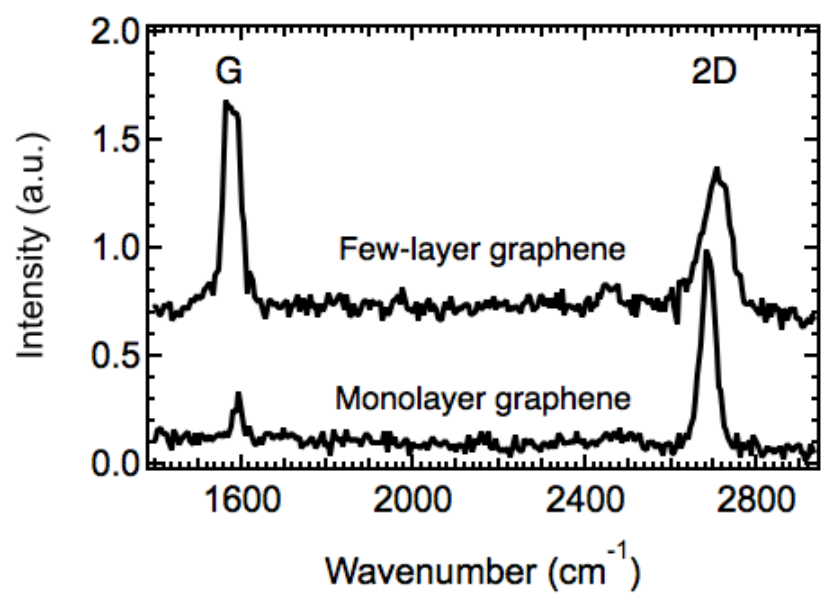

Figure 1.4: Raman spectra of monolayer graphene and few-layer graphene.

layers following the preliminary identification under the optical microscope. For optically active 2D materials, photoluminescence and absorption spectroscopy is also used which will be described later in chapter 2. In our lab, Raman spectroscopy is most commonly used to confirm the thickness of graphene. Raman spectroscopy has been widely used to identify molecules with its vibrational and rotational modes [19]. However, recently, it has been an essential technique to characterize the number of layers and strain in 2D materials [20]. Most of the photons incident at the atoms of a crystal are scattered elastically with the same wavelength. However, a fraction of the light is scattered inelastically by the atoms of the crystal which are excited to higher vibrational or rotational energy levels. This results in a slight shift in wavelength of some of the incident photons. This effect was first discovered by C. V. Raman and K. S. Krishnan [21]. The Raman spectra has unique signatures for different layer thicknesses which aids to establish the number of layers in the materials thus making it a valuable tool in the $2 \mathrm{D}$ laboratory. The energy difference between the incident light and the scattered light lets us estimate the energy of the phonon in the crystal. A phonon is the elementary vibrational mode of atoms in the crystal lattice. A characteristic Raman 
spectra of a monolayer graphene is presented in Fig. 1.4. The phonon energy is often expressed in terms of wavenumbers in units of inverse centimeters $\left(\mathrm{cm}^{-1}\right)$. The Raman spectra is characterized by two principle bands: the G and the 2D. The G (2D) band is the sharp band that appears around $1590 \mathrm{~cm}^{-1}\left(2700 \mathrm{~cm}^{-1}\right)$ in the spectrum of graphene resulting from primary in-plane vibrational mode (a second order effect of a different in-plane vibration) [22]. The differences in the band positions, shapes of the $\mathrm{G}$ band 2D bands and the relative intensity of these bands makes the Raman effect capable of determining layer thickness with atomic resolution [23]. For example, as shown in Fig 1.4, one of the striking feature in the Raman spectra of monolayer graphene is that the intensity ratio of the $2 \mathrm{D}$ peak with respect to the $G$ peak will always be close to two. However, for few layer graphene it is less than one.

\subsection{Hexagonal Boron nitride}

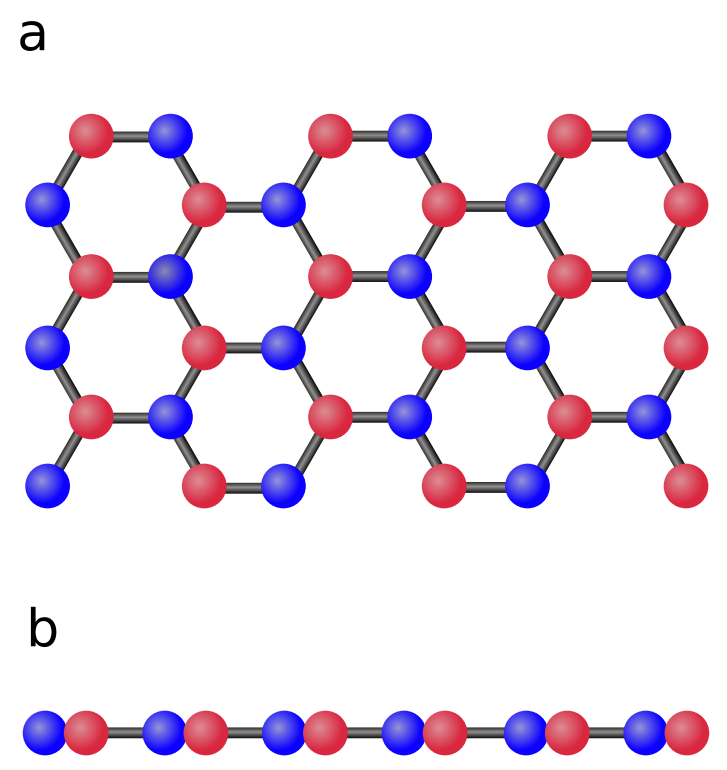

Figure 1.5: h-BN lattice: Ball and stick model of h-BN a. Top view b. Side view. 
Following graphene, monolayer hexagonal boron nitride (h-BN) was studied, as it was predicted theoretically that depositing graphene on h-BN would introduce a finite band gap in graphene [7]. Boron nitride $(\mathrm{BN})$ is a III-V compound that can exist in cubic, or wurtzite crystalline structures but h-BN has the most attention in recent times due to its layered form like graphite. Monolayer h-BN has structural properties similar to graphene. For eg, in Fig. 1.5, a schematic of a single layer of h-BN is shown where the $\mathrm{B}$ and $\mathrm{N}$ atoms (blue and red spheres, respectively) are arranged in a honeycomb structure similar to graphene in Fig 1.1. Its lattice constant is $2.5 \AA$ is which is only $1.8 \%$ larger than graphene [24]. However, the electronic properties are quite different: $\mathrm{BN}$ is an insulator with a gap of $\sim 5 \mathrm{eV}-6 \mathrm{eV}[25]$ instead of a conductor. It is this property that makes it one of the promising dielectric materials for integration with hybrid 2D optoelectronic devices. Significant research on h-BN concluded that it is an ideal substrate for 2D materials based electronics [26]. These experiments show that graphene supported on conventional substrates like $\mathrm{SiO}_{2}$ is very uneven as it conforms to the roughness of the substrate. This creates lots of wrinkles on the monolayer which restrains the capabilities of the device. Moreover the surface of these substrates usually have lots of impurities that can act as charge traps, significantly suppressing the carrier mobility of the electronic device. h-BN, on the other other hand, is atomically flat without any charge traps providing a perfect substrate for supporting devices based on atomically thin materials. Moreover, it has a dielectric constant of $\sim 3-4$ and a breakdown electric field of $1 \mathrm{~V} / \mathrm{nm}$ providing a very efficient gate insulating layer [27].

In addition to its insulating properties, h-BN is chemically inert in a wide variety of acids, solvents, and oxidizers and is also transparent in a wide range of wavelengths [28]. So, recently, it is also used as an ideal layered material for 
encapsulation and capping of various other 2D materials to protect them from oxidation or other atmospheric effects [29].

Mechanical exfoliation is, again, the most common method to produce high quality samples. In this work, few layer h-BN is mechanically exfoliated from it's bulk (ordered from HQ graphene). It has been used for encapsulation of devices as well as a dielectric material in van der Waals heterostructure based optoelectronic devices relying on the field effect in chapter 4,5 and electrostatic doping in chapter 6.

\subsection{Transition metal dichalcogenides}

In 1985, after the discover of fullerenes [2], it was shortly realized that inorganic layered materials like molybdenum disulphide $\left(\mathrm{MoS}_{2}\right)$ and tungsten diselenide $\left(\mathrm{WSe}_{2}\right)$ can also form similar closed cage structures [30, 31]. These materials are compounds made of transition metals and the chalcogen subgroups in the periodic table. Similarly, following the discovery of carbon nanotubes [1], inorganic nanotubes based on transition metal dichalcogenides were also prepared and characterized $[32,33]$. Therefore, after the discovery of graphene it seemed natural for scientists to explore semiconducting analogues of graphene in the form of layered inorganic materials. Among them, the layered TMDCs exhibit intriguing electronic and optical properties. Similar to graphene, several new properties of these TMDCs were discovered after their first isolation in 2005 [34] using the scotch tape technique.

Transition metal generally refers to elements in the d-block of the periodic table where the highest energy electrons reside in the d-orbital. Chalcogen, also known as the oxygen family, forms the group 16 of the periodic table. In this thesis, 
we mainly focus on the optical properties of some of the monolayer TMDCs like $\mathrm{WSe}_{2}, \mathrm{MoSe}_{2}$ and $\mathrm{MoS}_{2}$. This section will give a brief overview of some of the characteristics of the studied TMDCs.

\subsubsection{Crystal Structure}

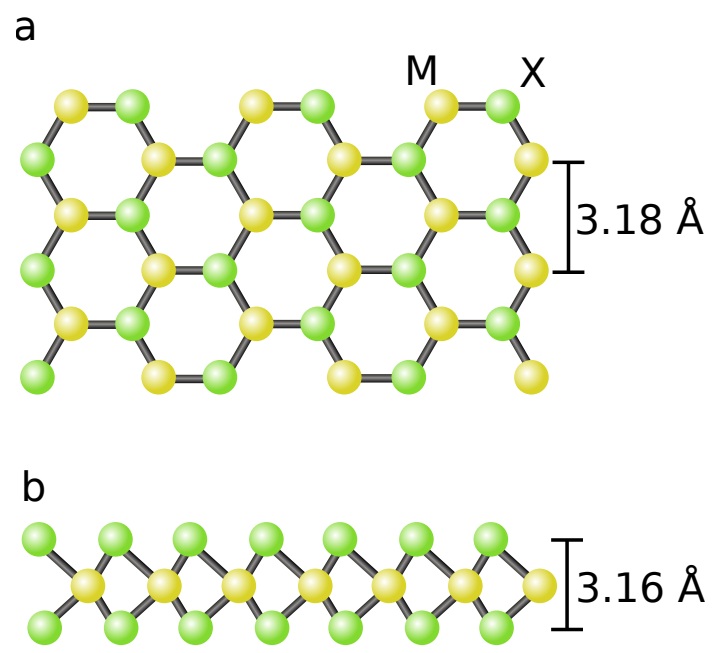

Figure 1.6: Monolayer TMDC lattice: Ball and stick model of a TMDC monolayer a. Top view b. Side view.

Transition metal dichalcogenides (TMDCs) have the general chemical formula $\mathrm{MX}_{2}$, where $\mathrm{M}$ can be a transition metal like molybdenum, Mo, or tungsten, W, and X can be chalcogens like sulfur, S, or selenium, Se. The crystal structures of these compounds are very similar to each other and consists of a $\mathrm{M}$ atom sandwiched by two X atoms. A schematic of a single layer TMDC is shown from the top and side view in Fig 1.6. X atoms are presented in green and $\mathrm{M}$ in yellow. In Fig 1.6a, the hexagonal structure similar to graphene is evident from the top view. However, any lattice point appearing to be filled by a single chalcogen atom is actually two chalcogen atoms one atop the other in a trigonal prismatic configuration. This is evident in the side view in Fig 1.6b where each TMDC 
monolayer is three atoms thick where a layer of $\mathrm{M}$ atoms is sandwiched by two layers of X atoms. The vertical spacing between two chalcogen in a monolayer is about $3.16 \AA$ [35]. Bulk crystals are formed by vertical stacking of these single layers that are held together by van der Waals forces similar to graphene. In the bulk, the orientation of the atoms are such that the chalcogens of one layer are aligned with the transition metal of the next layer in a $2 \mathrm{H}$ stacking form [36]. The interlayer spacing has been measured to be $6.5 \AA$ [37].

\subsubsection{Band structure}

a

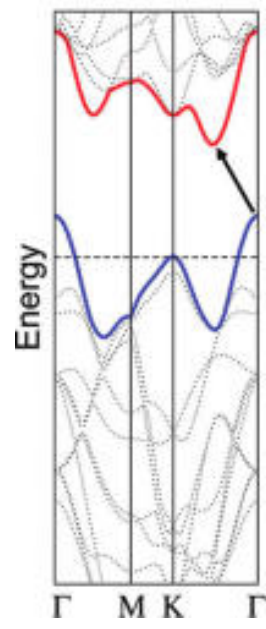

b

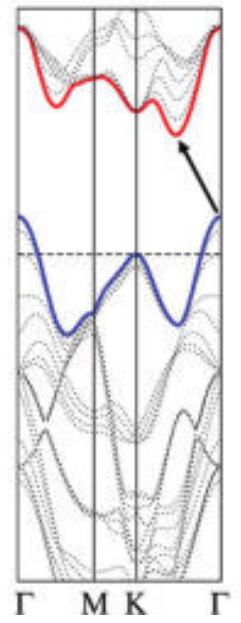

c

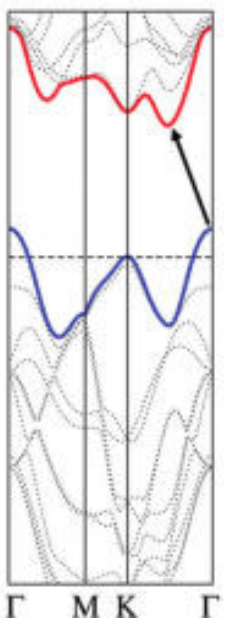

d

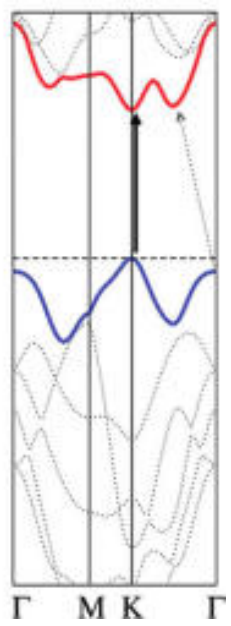

Figure 1.7: Electronic band structure of a. bulk b. quadralayer c. bilayer and $\mathbf{d}$. single layer $\mathrm{MoS}_{2}$. The tranistition from indirect to direct band gap is observed (left to right) [38].

The electronic band structure presents the range of allowed energies for an electron in the solid. In this section, we will present the electronic band structure of the TMDCs. Quite a few of the optical properties is dictated by the band structure of the material. Therefore, before we dive into the optical properties of 
the TMDCs in the next chapter it is important to understand some of the basics of the band structure in these materials.

The TMDCs have a layer dependent band structure. In the bulk form, it has an indirect band gap. However, it transitions to a direct band gap when the material is thinned down to a monolayer. This is because with reduced layer thickness, the indirect band gap becomes larger with increase in confinement, while the direct excitonic transition does not change. Thus, the lowest energy transition becomes the direct transition for the case of a monolayer. The electronic structure of TMDCs has been calculated by several theory groups to show the above mentioned transition from indirect to direct band gap semiconductor [38, 39].

In Fig. 1.7, the calculated band structure of an exemplary TMDC $\left(\mathrm{MoS}_{2}\right)$ using density functional theory is presented for (a) bulk, (b) quadralayer, (c) bilayer and (d) monolayer as reported by Spelndiani and colleagues [38]. The lower band is called the valence band which is filled with electrons for a semiconductor. The upper band is the conduction band which is is empty unless doped externally. The electrons in the valence band have limited motion within the band as all of the energy states are filled. Electrons in the conduction band are able to move fairly easily as the majority of the band is unfilled. The band structure reveals the available transitions between the valence and conduction bands. For the case of graphene, the conduction and valence band are connected at the $\mathrm{K}$ point allowing electron to move from valence to conduction band quite easily as there are no forbidden energy for the carriers. The TMDCs have a finite band gap. It is the energy difference between the valence band maximum and conduction band minimum. Generally, transitions can be made between any valence band local maximum and any conduction band local minimum. In Fig 1.7, the lowest energy transitions are indicated by solid black arrows in the band structure of $\mathrm{MoS}_{2}$. 
Owing to the strong hybridization between the d-orbitals of the metal atoms, the bulk TMDC exhibits an indirect band gap originating from transition between the valence band maximum at the $\Gamma$ point to the conduction band minimum halfway between the $\Gamma$ and $K$ point as shown in Fig. 1.7. The indirect transition is strongly affected by the presence of adjacent layers as the valence band maximum states near the $\Gamma$ point are a linear combination of the $d$ and $p$ orbitals of the metal and chalcogen atoms. However, the smallest direct band gap originates from the $d-d$ metal orbital transitions at the $K$ (and $K^{\prime}$ ) point in the Brillouin zone [40]. The direct transition is not affected by the interlayer interactions because the metal $d$ orbitals are sandwiched by the chalcogen layers. Hence, the indirect band gap exhibits strong dependence on the number of layers unlike the direct band gap (Fig. 1.7 a-d). Similar behavior of the band is present in others TMDCs such as $\mathrm{MoSe}_{2}, \mathrm{WS}_{2}$ and $\mathrm{WSe}_{2}$. The band gap makes the TMDCs favorable for not only optics which will be presented later in the thesis but also for electronic applications. High mobility field effect transistors based on TMDCs [41] and flexible photovoltaics [42] are few examples from recent reports.

\subsubsection{Preparation and identification}

Monolayer TMDCs can be either exfoliated from bulk or grown via CVD for large scale production. For the purpose of this thesis, monolayer flakes were mechanically exfoliated from bulk. The bulk material for $\mathrm{WSe}_{2}$ and $\mathrm{MoSe}_{2}$ devices studied in chapters 3-7 were bought from Nanoscience instruments store and HQ Graphene and $\mathrm{MoS}_{2}$ in chapter 7 was from SPI supplies. The monolayers are first identified by optical contrast under a microscope. The flake thickness can be confirmed using the same techniques used for graphene like AFM, TEM or by measuring the phonon modes by Raman spectroscopy. In addition, probing the 
excitonic transitions by optical techniques like photoluminescence and reflection contrast spectroscopy are also widely used for identification of the TMDCs and characterizing them. These will be explored in details in chapter 2. Below we will discuss the Raman spectra from some of the TMDCs.

\section{Raman spectra of monolayer TMDCs}
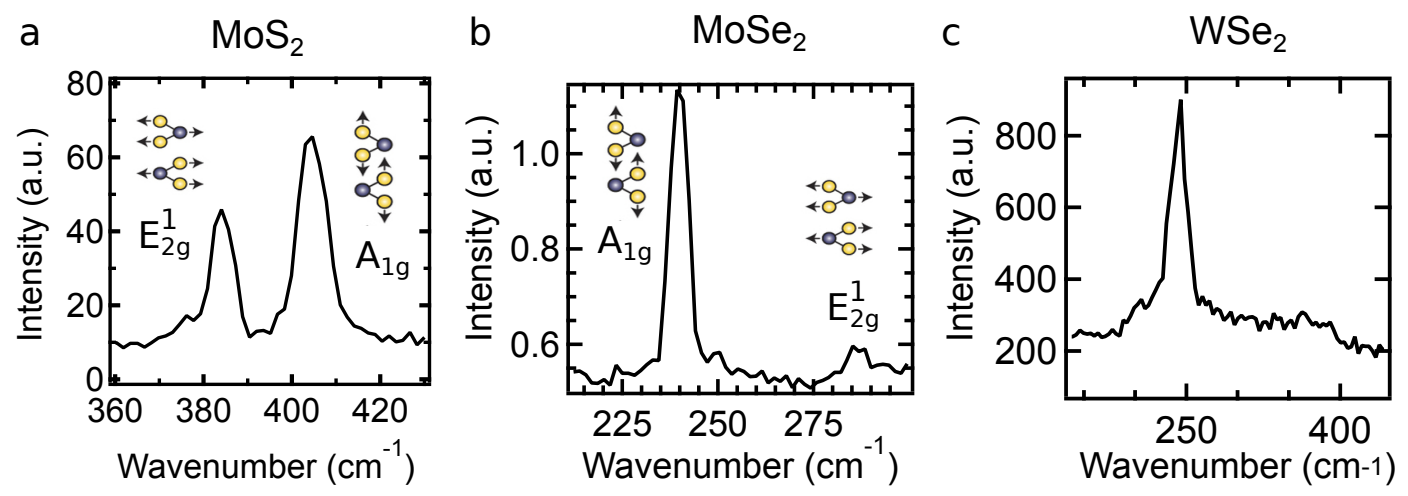

Figure 1.8: Raman spectra of a. $\mathrm{MoS}_{2}$ b. $\mathrm{MoSe}_{2}$ and c. $\mathrm{WSe}_{2}$

The Raman modes in TMDCs have been thoroughly measured and reported [43]. The Raman spectra from the exemplary monolayer flakes studied in this thesis are presented in Fig. 1.8 and compared to literature. In $\mathrm{MoS}_{2}$ (Fig 1.8a), we observe two Raman modes: the $E_{2 g}^{1}$ and $A_{1 g} . E_{2 g}^{1}$ is an in-plane vibrational mode, and $A_{1 g}$ is an out-of-plane vibrational mode. With increasing sample thickness, the $E_{2 g}^{1}$ mode redshifts, and the $A_{1 g}$ mode blueshifts [43]. One useful metric for distinguishing the thickness of the flake is the difference in frequency of these two vibrational modes. In $\mathrm{MoSe}_{2}$ (Fig 1.8b), the $A_{1 \mathrm{~g}}$ mode occurs at about $240 \mathrm{~cm}^{-1}$ and the $E_{2 g}^{1}$ mode is found around $287 \mathrm{~cm}^{-1}$ for monolayer. Trends similar to $\mathrm{MoS}_{2}$ are observed in the two modes as a function of sample thickness [43]. In $\mathrm{WSe}_{2}$ (Fig $1.8 \mathrm{c}$ ), the $E_{2 g}^{1}$ and $A_{1 g}$ modes are nearly degenerate so only one peak 
is visible in the Raman spectra. However, the two peaks are resolved in the bulk where they are shifted in energy [43].

\subsection{Van der Waals heterostructures}

Although the diversity of the different materials has been described in the last few sections, there is one property that all of these above materials have in common: their weak interlayer bonding via van der Waals force in the bulk form. This makes it natural to think if one can deploy these weak forces to bond the monolayers of the materials having different properties. This concept of 'LEGO in the atomic scale' was first proposed by Geim et al. [44]. Different monolayers with different properties can be stacked together to form a brand new material or an optoelectronic device with customized properties. This is a borrowed from an old concept that has been used to make semiconductor quantum wells (for example, AlGaAs/GaAs/ALGaAs) using molecular beam epitaxy. However, the need for lattice matching the layers in the case of van der Waals epitaxy are relaxed due to weak interlayer forces that hold the stack together. This makes it very simple and inexpensive to prepare heterostructure based devices. van der Waals heterostructure are built for studying field effect transistors, tunneling diodes, photovoltaics, LEDs and even interlayer excitons [45, 46, 47]. Moreover, the van der Waals force not only helps to build heterostructures of $2 \mathrm{D}$ material but also lets it integrate with other dimensional structure like nanowires and quantum dots. In this thesis, we use this concept to build field effect devices for studying quantum confined Stark effect and charging via electrostatic doping (chapters 4 - 6). 

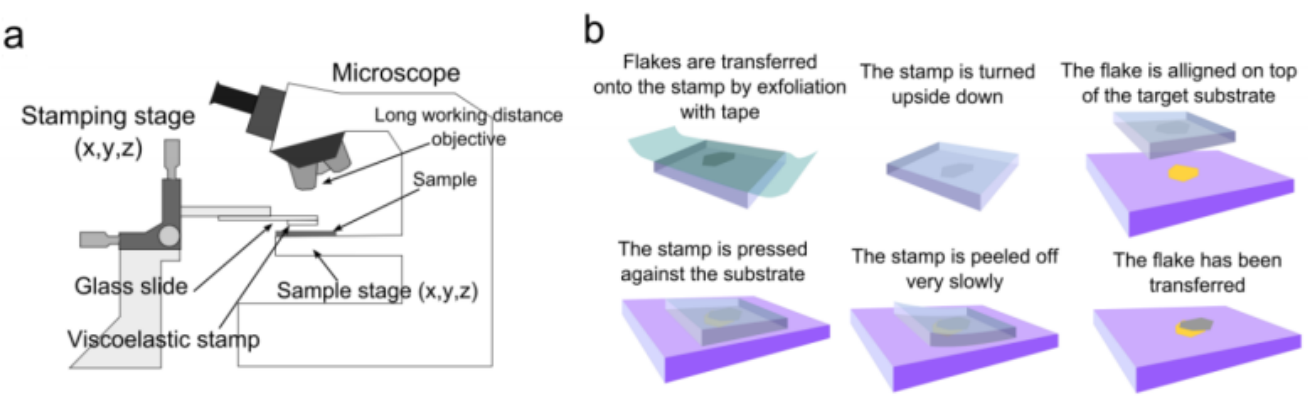

Figure 1.9: Dry transfer technique: a. The all dry stamping setup and b. process flow [48].

\subsubsection{Assembling the layers}

In this section, a general description of the assembly technique of a van der Waals heterostructure will be provided. A detailed description of the particular devices studied in this thesis will be provided in the later chapters. A van der Waals heterostructure is assembled by the commonly known dry transfer technique [48]. The stamp used for the transfers in our lab is a thin layer of commercially available viscoelastic PDMS gel (Gelfilm PF-X4-6.5 from Gelpak) which is adhered to a coverslip to facilitate its handling. Polydimethylsiloxane or PDMS is a silicon based organic polymer. The two-dimensional crystals to be transferred are deposited onto the viscoelastic layer by mechanical exfoliation of the bulk layered crystal with semiconductor dicing tape from Semicorp. The surface of the stamp is then inspected under the optical microscope to select the thinner flakes from the contrast. Once a monolayer flake has been identified, the target substrate (on which the flake is to be transferred) is fixed using double-sided tape on the XYZ microscope stage having a large working distance objective (Fig 1.9a). The PDMS stamp is attached to a three axis manipulator with the flakes facing towards the target substrate. As the stamp is transparent, one can see the monolayer flake 
through it and thus it is possible to align the desired flake on the target surface where one wants to transfer the flake with sub-micrometer resolution. This process can be repeated to transfer multiple flakes on the same target.

\subsection{Thesis outline}

In this chapter, we have introduced the different variety of $2 \mathrm{D}$ materials that have been either studied or used for fabricating various devices based on van der Waals heterostructure for this thesis. The crystal structure, methods of isolation and identification have been described for graphene, TMDCs and h-BN. Lastly the concept of van der Waals heterostructure and their assembly technique has been discussed. The outline of the rest of the thesis is presented below.

In chapter 2, a brief introduction of excitons in $2 \mathrm{D}$ materials and their relevant properties are discussed for the understanding of this thesis. A review of the exciton dominated optical properties through several spectroscopic techniques are presented. Further, generation and properties of different form of excitons like neutral and charged excitons, localized excitons, interlayer excitons and the valley properties of the $2 \mathrm{D}$ excitons are also discussed. These will not only help to compare the results of $2 \mathrm{D}$ delocalized excitons with the $3 \mathrm{D}$ localized excitons presented in chapters $3-6$ but also provide an understanding into the working principles of the devices presented in chapter 7 .

In chapter 3, the discovery and the various photophysical properties of the excitons localized in the imperfections of the monolayer TMDCs are outlined. These optical properties are studied using low temperature micro-photoluminescence measurements. Intensity autocorrelation measurements have been performed to confirm the single photon nature the emitters in monolayer $\mathrm{WSe}_{2}$. The dependence 
of the emission properties on different parameters like excitation power, temperature and gate voltage have been studied. Further, polarization and magnetic field resolved photoluminescence measurements confirmed that most of the localized emitters are derived from the host excitons that are trapped in an asymmetric confinement potential in the crystal.

Chapter 4 presents the possibility of building electrically tunable van der Waals heterostructure encapsulating the localized emitters. Such devices demonstrated tunable transition from these localized emission states via the quantum confined Stark effect. A range of values for the components of permanent dipole moment and polarizability were extracted suggesting the different influences of the environment, shape and size on individual localized emitters.

Chapter 5 outlines the extent of modulation of the fine structure splitting present in the single excitons of these localized emitters. This shows that the symmetry properties of the confinement potential that dictates the electron-hole exchange interaction for localized excitons can be manipulated by using a van der Waals heterostructure based field effect device. Partial recovery of the circular polarization due to the valley properties of the excitons can also be recovered using this technique.

Chapter 6 discusses the formation of charged excitons in the localized states of $\mathrm{WSe}_{2}$ embedded in a van der Waals heterostructure that are capable of electrostatic control of the charge carriers. The single neutral excitons in the emitters can form charged excitons on applying the electrostatic field. The charged nature of the excitons are confirmed via power and polarization dependent photoluminescence measurements.

In chapter 7, three different devices based on integrated optoelectronics and nanophotonics with 2D materials are presented. Section 7.1 presents the in- 
tegration of a graphene photodetector with a single bow-tie nanoantenna. The antenna concentrates the electromagnetic field from laser excitation which resulted in a photoresponsivity of the order of tens of $\mathrm{nA} / \mu \mathrm{W}$ from a device under zero bias. In section 7.2, a monolayer $\mathrm{MoS}_{2}$ photodetector is presented as a near field photodetector via coupling with nanowire plasmons even under a gain-free configuration. Finally, in section 7.3, the strong coupling of cavity photons with the neutral and charged excitonic resonances of monolayer molybdenum diselenide $\left(\mathrm{MoSe}_{2}\right)$ is discussed. The device is a monolayer TMDC embedded in a monolithic optical microcavity based on distributed Bragg's reflectors. The many body interactions lead to multiple polaritonic resonances and inverted band dispersion for the lowest trion derived branch. These work connects the fields of nanophotonics, nanoplasmonics, and atomically-thin materials and provides a stepping stone for more complex nanoscale circuitry. 


\section{Chapter 2}

\section{Flatland excitons}

This chapter gives a brief overview of the different properties of exciton in the TMDC flatland. We will introduce some of the optical properties of the TMDCs that are mainly dominated by the exciton resonances through photoluminescence and reflectance spectroscopy. The concept and the creation of charged excitons in TMDCs will be established. Further, a discussion on the valley properties of the excitons will be also provided. While there are many other interesting characteristics of the $2 \mathrm{D}$ excitons, we will only introduce few concepts in this chapter that are relevant to understanding and comparing the results in the remainder of this thesis.

\subsection{Excitons in 2D}

In semiconductors, electrons in the valence band can be excited to the conduction band by incident photons with energy larger than or equal to the band gap. The vacancy left behind in the valence band is a positively charged quasi-particle called a hole. If the Coulomb forces are strong enough between the electron and hole, they can form a bound state called an exciton. It is the most fundamen- 
tal optical excitation found in semiconductors that can be created or annihilated upon the absorption or emission of a photon. It has its own internal fine structure with hydrogen-like excited energy states, potentially providing a ground for studying fundamental physics and future devices with excitonic circuits [49]. In typical bulk semiconductor crystals, such as silicon or GaAs, the large dielectric screening and small effective mass of the electron and hole quasiparticles result in small exciton binding energies, only up to tens of meV [50]. Thus, the bound behavior of an exciton is insignificant compared to the thermal fluctuations unless cooled down to low temperatures. However, in single-layered TMDCs, the carriers can form strongly bound excitons [51], charged excitons [52], and excitonic molecules [53]. The excitons in TMDC have a binding energy that is almost two orders of magnitude larger than traditional III-V semiconductors due to the strong Coulomb interactions arising from low dimensionality, reduced dielectric screening and the large effective masses of electrons and holes [54]. For example, in monolayer TMDCs exciton binding energy up to $700 \mathrm{meV}$ have been observed with effective electron mass of around 0.2-0.4 $m_{0}$ [55] and exciton Bohr radius of $\sim 1 \mathrm{~nm}$ [54] which is also one order of magnitude smaller than in GaAs. Their radiative recombination lifetime is of the order of picoseconds [56]. Furthermore, excitons in TMDCs are strongly influenced by the strong spin-orbit coupling producing a large splitting the valence band [57]. This results in the formation of two excitonic transitions, usually referred to as A and B as shown in Fig 2.4b, associated to direct optical transitions between band edges at the $\mathrm{K}$ points [58]. The giant magnitude of the valence band spin-orbit interaction $\left(\Delta_{V B}\right.$, Fig. 2.6) in ultrathin TMDC layers is predicted to be from $\sim 140 \mathrm{meV}$ to $460 \mathrm{meV}$. In addition, there is also a relatively small but significant splitting in the conduction band $\left(\Delta_{C B}\right)$ with magnitudes up to $38 \mathrm{meV}$ depending on the material [59]. Most 
of the excitonic phenomenon is probed using photoluminescence and reflectance spectroscopy which will be described in the following subsection.

\subsubsection{Photoluminescence}

Photoluminescence (PL) is the emission of a photon due to the recombination of an electron-hole pair that is optically created. An electron is excited from the valence band to the conduction band when the material is optically excited having energy larger than the band gap $\left(\mathrm{E}_{g}\right)$ of the material. Electrons and holes relax to the bottom of their bands and recombine leading to the creation of a photon with energy $\left(\mathrm{E}_{e x}\right)$ around the band gap subtracted from the exciton binding energy $\left(\mathrm{E}_{B E}\right)$ as shown in Fig. 2.1a.

\section{Material and layer dependence}
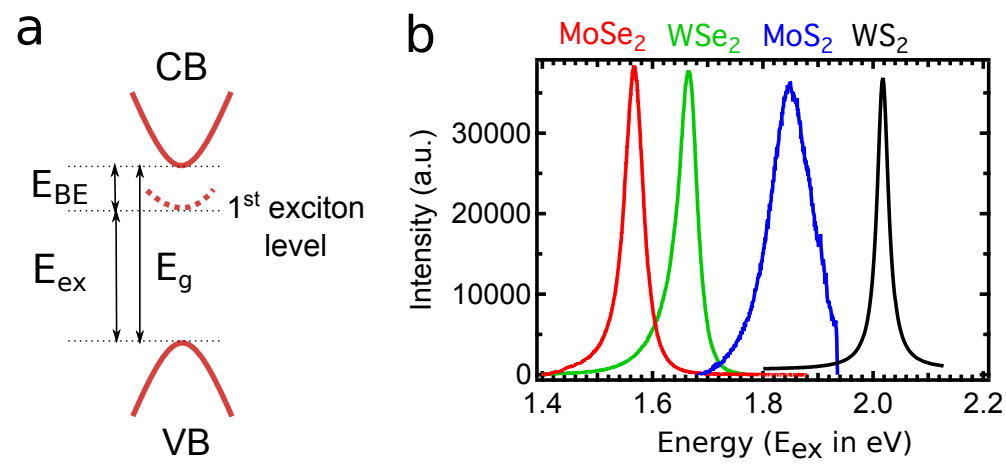

Figure 2.1: Photoluminescence: a. Band diagram of a semiconductor presenting the respective energies related to the material band gap $\left(\mathrm{E}_{g}\right)$, exciton binding energy $\left(\mathrm{E}_{B E}\right)$ and the optical transition energy $\left(\mathrm{E}_{e x}\right)$ due to exciton recombination b. Photoluminescence spectra from monolayers of four different TMDCs at room temperature. 
The photoluminescence from a monolayer TMDC is dominated by the A exciton PL, as shown in Fig 2.1b. Different TMDC emit at different energies depending on their band gap and exciton binding energies.
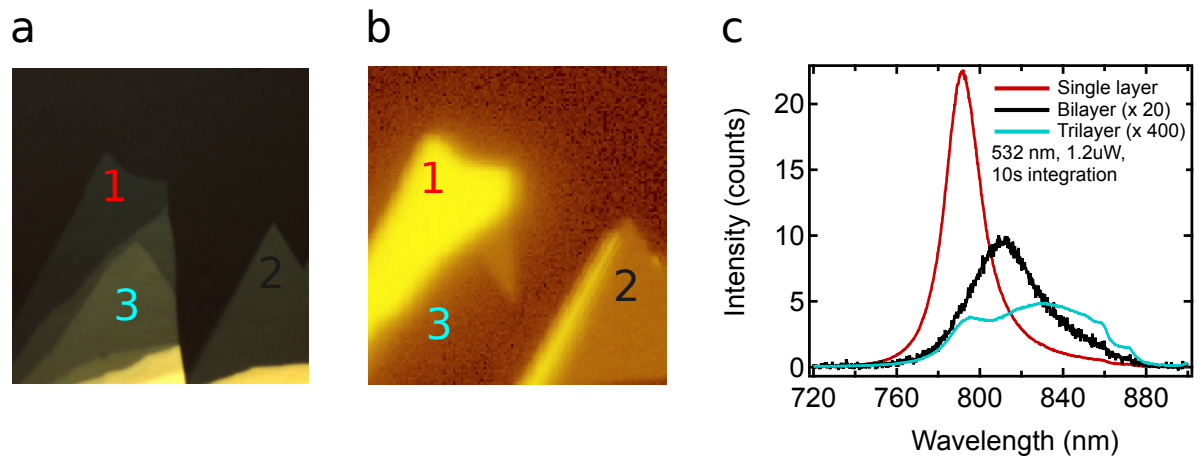

Figure 2.2: Layer dependent PL: a. Optical micrograph image of an exfoliated $\mathrm{MoSe}_{2}$ flake on a substrate. b. Fluorescence map of the same flake as (a). The numbers represent the layer number of the flake $\mathbf{c}$. Photoluminescence spectra of single, bilayer and trilayer $\mathrm{MoSe}_{2}$ at room temperature.

TMDCs also exhibit a layer dependence band gap as discussed in chapter 1 . For the case of monolayer, the bottom of the conduction band aligns with the top of the valence band, ie, they occur at the same k-vector in the Brillouin zone. The strong photoluminescence for the case of monolayer TMDCs is a result of this direct band gap transition. The PL quantum yield can even approach 100\%, with the assistance of chemical treatments [60]. However, when they lie on different $\mathrm{k}$-vectors, this leads to an indirect band gap where a phonon assisted transition is needed for the radiative recombination process and, therefore, the efficiency is low. This is exhibited by the layer dependence photoluminescence for $\mathrm{MoSe}_{2}$ (Fig. 2.2c). Figure 2.2 shows the fluorescence map and photoluminescence spectrum of one, two, and three layers of exfoliated $\mathrm{MoSe}_{2}$. The observed emissions from monolayer and bilayer $\mathrm{MoSe}_{2}$ exhibit a single prominent maximum at $\sim 792 \mathrm{~nm}$ and $\sim 807 \mathrm{~nm}$, respectively. The PL intensities from the monolayer material are $\sim$ 
40 times stronger than those of the bilayer material. The emission from the trilayer material shows two prominent maxima, one near the direct A exciton transition and a broader one representing the indirect transition. The PL intensity decreases again by about an order of magnitude as compared to the bilayer. The observed layer dependent PL for our $\mathrm{MoSe}_{2}$ samples are in excellent agreement with the known direct and indirect exciton emission from recent experimental results [61].

\section{Temperature dependence}
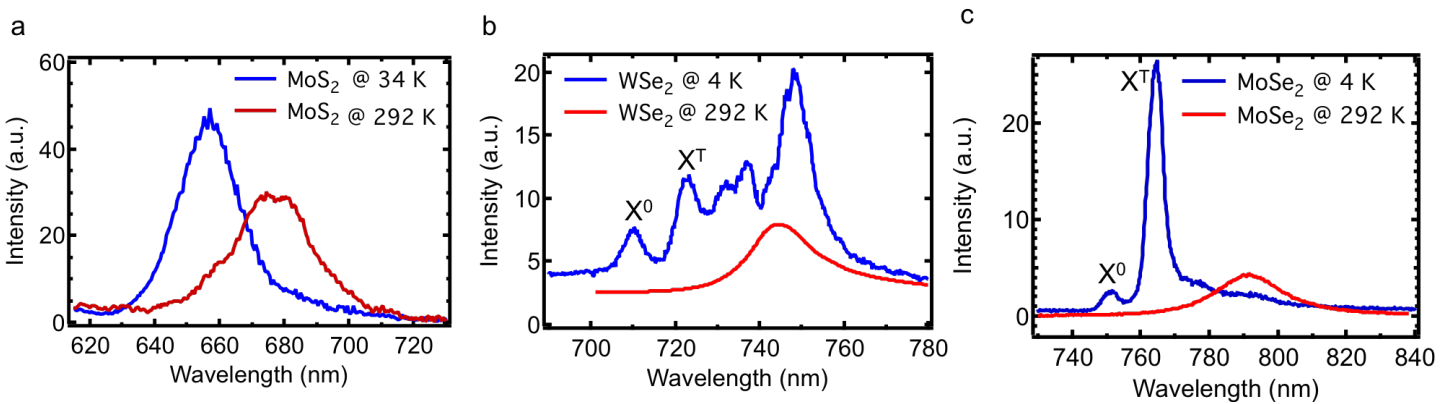

Figure 2.3: Temperature dependent PL spectra of a. $\mathrm{MoS}_{2}, \mathbf{b} . \mathrm{WSe}_{2}$ and c. $\mathrm{MoSe}_{2}$.

TMDC absorption and emission spectra are closely related to the environment temperature [56]. Exciton in TMDCs redshift as the temperature increases (Fig. 2.3), which is attributed to the temperature-induced variation of lattice parameters. At low temperature, the trion peak $\left(X^{T}\right.$, Fig. $\left.2.3 \mathrm{~b}-\mathrm{c}\right)$ can also be resolved. The trion is another kind of exciton species which is a combination of an exciton bound to an electron or hole depending on the initial doping of the material. In addition, there is a broadening in the exciton and trion linewidths as the temperature increases. This temperature-dependent broadening is attributed to the increase of the exciton-phonon scattering rates [62]. 


\subsubsection{Reflectance}
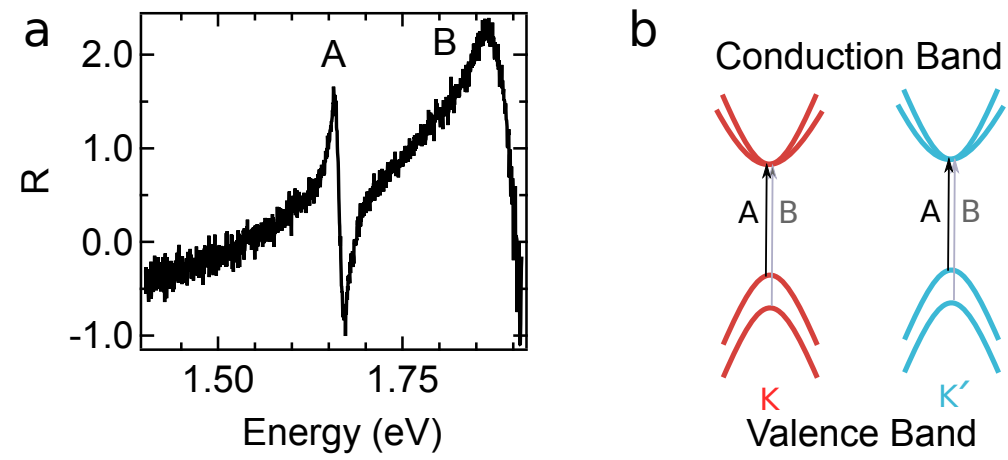

Figure 2.4: Reflectance: a. Reflectance spectra of monolayer $\mathrm{MoSe}_{2}$ at $4 \mathrm{~K}$. b. Illustrations of the conduction and valence bands at the $K$ and $K^{\prime}$ valleys of a typical monolayer TMDC, showing the A and B exciton transitions.

Reflection contrast or reflectance $(\mathrm{R})$ is a type of measurement that encodes information about sample reflectivity and absorption. This information is, furthermore, related to the dielectric function of the material. To perform this type of measurement in our lab, the sample is illumination by a halogen source from Ocean Optics to acquire a spectra on the spectrometer camera $\left(R_{\text {flake }}\right)$. An additional spectra is taken on the background substrate $R_{\text {Substrate }}$ and normalized as follows:

$$
R=\frac{R_{\text {flake }}-R_{\text {Substrate }}}{R_{\text {Substrate }}}
$$

Fig 2.4a shows a characteristic differential reflectance spectra of a monolayer $\mathrm{MoSe}_{2}$ at $4 \mathrm{~K}$. The spectrum is dominated by the A and B exciton resonances. The $\mathrm{A}$ and $\mathrm{B}$ excitons belong to the excitons in the two valleys of the valence band at the $\mathrm{K}$ and $\mathrm{K}^{\prime}$ points respectively, as illustrated in Fig 2.4b. 


\subsection{Charged excitons}

One of the fundamental questions we need to address in any studies of 2D TMDCs is how the electrical and optical properties change with the change in Fermi level of the materials. This section is focused on the influence of the Fermi level (or carrier densities), on the optical properties of monolayer $\mathrm{WSe}_{2}$ as an exemplary TMDC. The scheme we use in our experiments is electrostatic doping. This is based on two terminal device where one electrode is connected to the flake and the other is connected to the backgate (degenerately doped $\mathrm{Si}$ ), that is separated from the flake by a layer of $300 \mathrm{~nm}$ oxide (Fig 3.8a). Electron-beam lithography (EBL) with poly(methyl methacrylate) (PMMA) as the resist, and electron-beam evaporation with the lift- off process were used to define the source/drain and gate electrodes. The metal contacts here are $5 \mathrm{~nm}$ chromium topped with $50 \mathrm{~nm}$ of gold. If an electric field is applied throughout a metal-oxide-semiconductor (MOS) structure, free or fixed charges will accumulate at the interface between the oxide and the semiconductor; such charges can effectively dope the semiconductor in the region near the interface, thus shifting the Fermi level of the semiconductor into the conduction or valence band. The electrical doping by this electrostatic gating technology has the capacity of changing the carrier density upto $10^{13} \mathrm{~cm}^{-2}$ [63]. This effect has been commonly used in electronic and optoelectronic devices to tune the conductivity as well as the electrical barrier in the devices.

Next, we will study the effect of the electrostatic doping on the excitons. The atomic thickness increases the interaction of exciton and charge carriers, forming tightly bound charged excitons, namely, trions. When applying back gate voltages on an exemplary monolayer $\mathrm{WSe}_{2}$ transistor, the PL spectra show obvious tuning with variant gate biases as shown in Fig 2.5c. The data in Fig $2.5 \mathrm{c}$ is taken at $4 \mathrm{~K}$. It shows various spectral features whose intensities strongly 

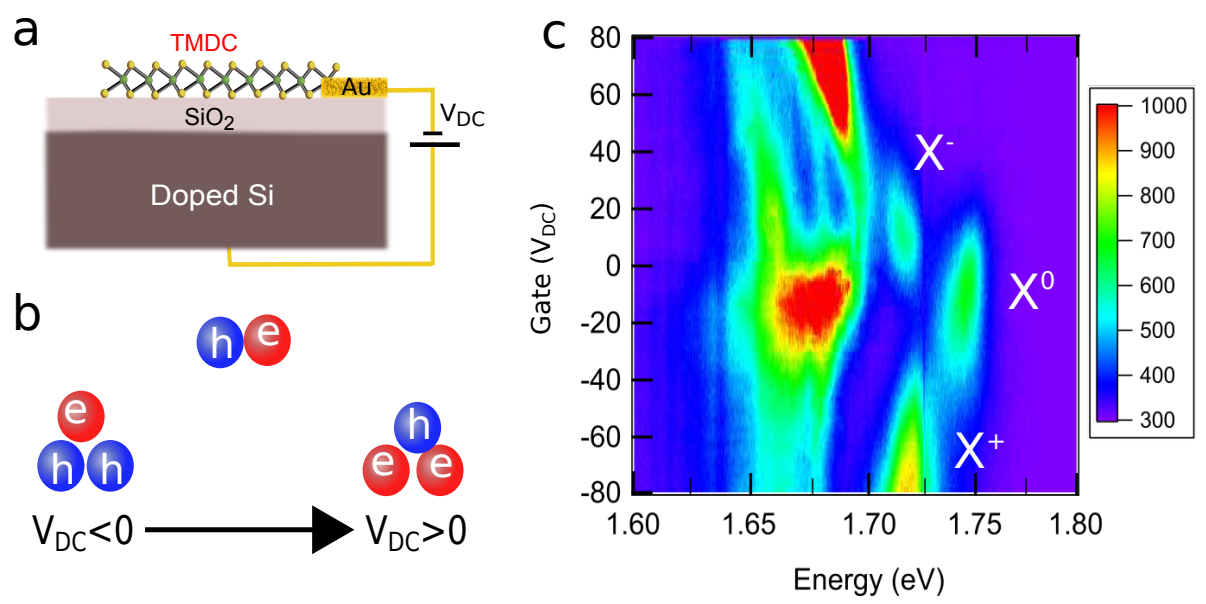

Figure 2.5: Charged excitons: a. Device geometry b. Illustration of the gate voltage dependent trion and exciton c. Evolution of the exciton peak's photoluminescence as a function of applied gate voltage $V_{D C}$

depend on the gate voltage. Near zero voltage, the peak emitting at the highest energy $\sim 1.75 \mathrm{eV}$ corresponds to the neutral exciton $\left(\mathrm{X}^{0}\right)$. As illustrated in Fig 2.5b, with large gate voltage of either sign, the neutral exciton disappear and second highest energy peaks corresponding to positively $\left(\mathrm{X}^{+}\right)$and negatively $\left(\mathrm{X}^{-}\right)$ charged exciton appears. The lower energy peaks beyond the trions are typically assigned to bound excitons related to defects and impurities. From Fig. 2.5c, the binding energy of trions can be directly extracted by taking the energy difference between the trion and neutral exciton. It varies as a function of gate voltage and ranges between $25-35 \mathrm{meV}$. The significant red shift in trion is likely due to the quantum confined Stark effect [64].

\subsection{Concept of Valleytronics}

Most modern electronic devices conceptualize the electron charge as the fundamental unit of information. However, it is also possible to build logic devices using 
other degrees of freedom of the electron. The spin degree of freedom is used for spintronic devices. Similarly, valley based electronic applications have recently attracted great interest where the extra valley degree of freedom is suggested as an information carrier [65]. This is relevant for materials whose whose conduction and valence band edges occur at two energy degenerate points ( $\mathrm{K}$ points) of the Brillouin zone. The two inequivalent point of the valley can be thought as bits in information processing. This gives rise to the concept of valleytronics.

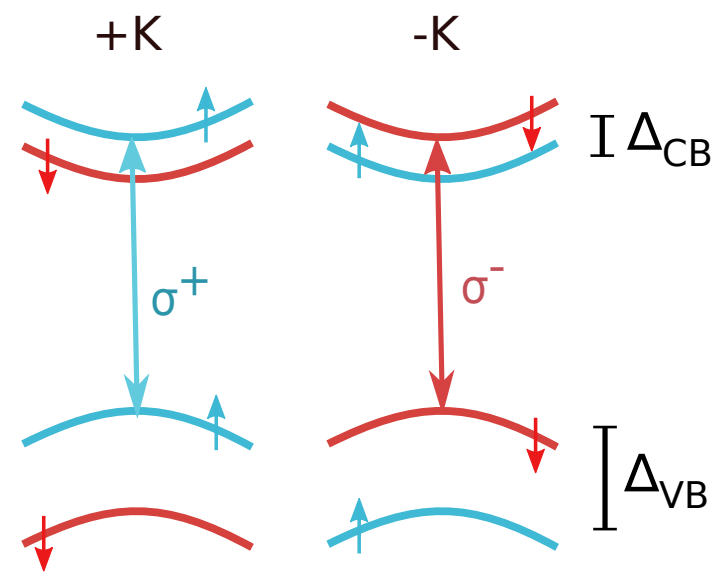

Figure 2.6: Schematic band structure of $\mathrm{W}$ based TMDCs near the $\pm K$ points showing the optical selection rules for the lowest energy (A exciton) transition. For the case of Mo based TMDC, the sign of the conduction band splitting is reversed. Within each valley, the spin orbit coupling breaks the spin degeneracy. The arrows denote up and down electron spin for the occupied states. $\sigma \pm$ represents the helicity of circularly polarized photons that couple to the optical dipole transitions. $\Delta_{V B}\left(\Delta_{C B}\right)$ represents the value of valence (conduction) band splitting due to spin-orbit coupling.

TMDCs provide an ideal platform for exploring valley pseudospin degrees of freedom. In monolayers, the metal and chalcogen atoms form a 2D hexagonal lattice that lacks spatial inversion symmetry (Fig. 1.6). The breaking of inver- 
sion symmetry and the spin-orbit interaction produces valley contrasting physical quantities and optical selection rules at the conduction and valence band edges located at $\pm K$ points in momentum space [66]. This is unlike bilayer TMDCs and graphene where both are centrosymmetric. Further, the conduction and valence band edges are at the $\pm K$ points are formed predominantly by the partially filled d-orbitals of the heavy metal atoms in TMDC monolayers that lead to strong spin-orbit coupling. This lifts the energy degeneracy of the spin states in the valence bands in the $\pm K$ valley by $100 \mathrm{~s}$ of mev $\left(\Delta_{V B}\right)$. The conduction band also has a small but finite spin-valley splitting $\left(\Delta_{C B}\right)$ with a magnitude of few tens of meV for different monolayers TMDCs. An important consequence of this spin-orbit coupling is that the spin-index becomes locked to the valley index at the band edges (Fig 2.6). Consequently, spin can be selectively excited through valley optical selection rule and intervalley scattering is suppressed due to a large $\mathrm{k}$ space separation between the two valleys. Further, the spin-split valence bands at the individual $\mathrm{K}$ valleys affords protection from both the spin and valley flips, as a result, long lifetime of valley polarization is expected. This valley contrast can be exploited to produce a valley Hall effect (carriers in different valleys flow to opposite transverse edges on application of an in-plane electric field) as well as allowing for the optical preparation, control, and detection of valley pseudospin polarization [65]. Therefore, information may be readily encoded not only by the spin up or down states of an electron (or hole), but also by the two momentum indices whether it resides in the $\mathrm{K}$ or $-\mathrm{K}$ valley. In the following section, results from polarization resolved and magneto-photoluminescence studies from both literature and our samples are discussed to examine the valley properties of the exciton. 


\subsubsection{Valley excitons: Polarization and Coherence}

In the polarization resolved PL measurements, circularly polarized laser excites electron-hole pairs selectively in a valley, which then form valley polarized excitons. Thus, excitons also have a binary valley pseudospin similar to free electrons and holes. As seen in Fig. Fig. 2.7a-b, stronger emission is observed in the copolarized spectra than the cross polarized spectra, indicating that, indeed, the photo-excited carriers preferentially formed excitons in the valley they were excited in creating a valley polarization [67]. Although this suggests an advantage for valleytronics based applications, yet any practical realization requires its depolarization timescales to be longer than the operation time of an external gate parameter. The two valley excitons are coupled by the electron-hole exchange Coulomb interaction that dominates the exciton valley depolarization process [68]. However, this mechanism becomes less effective for the case of trion [69] and interlayer excitons [70]. This indicates that applications of monolayer excitons in valleytronics might be limited to ultrafast timescales, whereas, trions and interlayer valley excitons in 2D heterostructures may be a better system to explore for valleytronic manipulation.

The creation and detection of valley coherence is another manifestation of valley optical selection rules. A coherent superposition of a left and right circularly polarized photon, i.e., a linearly polarized photon can transfer optical coherence into excitonic valley coherence. Linearly polarized PL from neutral excitons is observed in monolayer $\mathrm{WSe}_{2}$ with a polarization angle that always coincides with that of the linearly polarized excitation (Fig. $2.7 \mathrm{c}, \mathrm{f}$ ). Thus, the optically generated valley coherence survives the exciton formation and recombination processes, suggesting a long valley dephasing time [67]. 
a

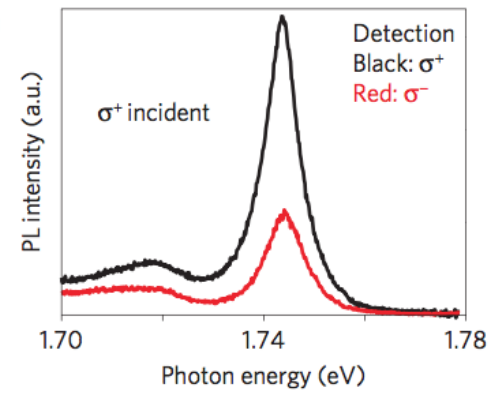

b

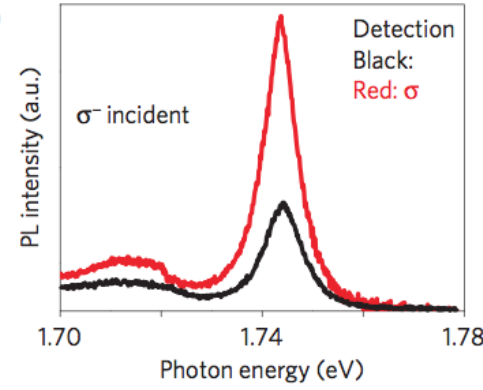

c

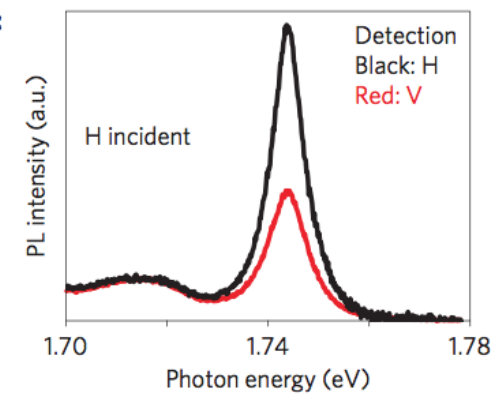

d $\mathrm{K} \quad-\mathrm{K}$<smiles>C[C]1CC1</smiles><smiles>C1CCC1</smiles>

$\sigma^{+}$ $\lim \rightarrow$

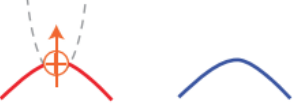

e

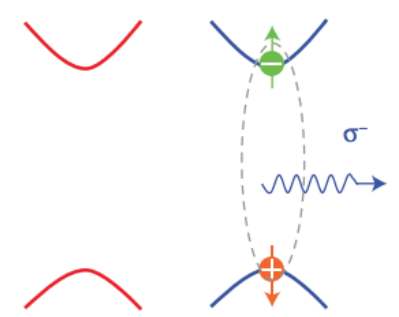

f

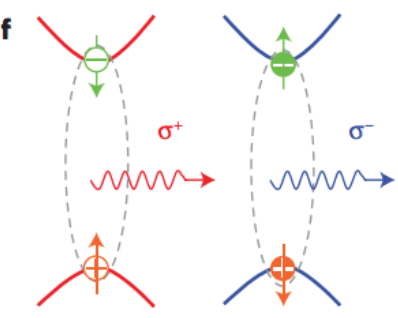

Figure 2.7: Polarization resolved PL: PL spectra from $\mathrm{WSe}_{2}$ under a. $\sigma^{+}, \mathbf{b} . \sigma^{-}$, c. linear excitation laser. d.-f. Transition for the given polarization from the respective valleys. $\mathrm{H}(\mathrm{V})$ represents horizontal (vertical) polarization axis of the linearly polarized light. [67]

\subsubsection{Valley Zeeman effect}

In analogy to the spin Zeeman effect, the valley magnetic moment of an electron in a monolayer TMDC also interacts with an external magnetic field, giving rise to a valley Zeeman effect. The valley Zeeman effect can be used to break the energy degeneracy of the two valleys, thus providing an additional degree of external 
a

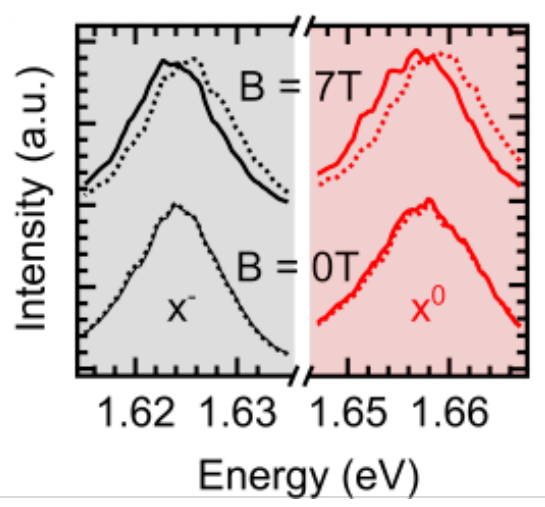

b

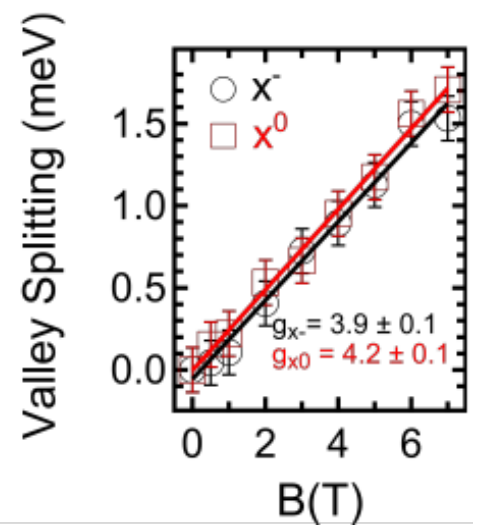

Figure 2.8: Valley Zeeman effect: a. Spectra of $\sigma^{-}$(dotted lines) and $\sigma^{+}$(solid lines) polarized exciton (black) and trion (black) PL for an applied magnetic field of $0 \mathrm{~T}$ (bottom) and 7T (top). Under an applied field the two cross polarized spectra split due to the valley Zeeman effect $\mathbf{b}$. Valley splitting as a function of magnetic field.

control over the valley pseudospins. This energy difference can be measured by performing polarization resolved magneto-optical photoluminescence [71, 72].

The normalized PL spectra of the neutral exciton and trion for $\mathrm{MoSe}_{2}$, for both valley pseudospin are plotted for given values of the applied magnetic field in Fig 2.8a. In the absence of the field, the two spectral lines overlap each other as the valleys are energy degenerate. As a static magnetic field is turned on, the $\sigma^{+}$polarized and the $\sigma^{-}$polarized peak split in energy. This is the valley Zeeman effect, and by measuring the splitting as a function of field one can calculate the valley magnetic moment (Fig 2.8b). The calculated magnetic moment in one of our TMDC monolayer is 4.2 and 3.9 for the exciton and trion respectively, which is extracted from a linear fit [73]. 


\subsection{D Excitons in the 2D}

Along with all the intriguing properties of TMDC excitons seen in the earlier sections, the recent discovery of quantum emitters in monolayer TMDCs $[74,75,76$, $77,78]$ has also focussed the attention of the solid-state quantum optics community towards these materials. Artificial ways of exciton localization in TMDCs from the top-down using lithographic masks [79] is challenging as accessing the strong confinement regime for TMDC quantum dot requires a nanofabrication length scale commensurate with the materials' small exciton Bohr radius $(\sim 1 \mathrm{~nm})$. Therefore, few natural ways would be to identify defects in the material that can localize excitons or leverage on strain-induced localized potential traps due to imperfections in the $2 \mathrm{D}$ crystal lattice. $\mathrm{WSe}_{2}$ was the first $2 \mathrm{D}$ material where single photon emitting centers owing to such localizations were discovered [75, 74, 76, 77, 78]. This discovery has stimulated the pursuit for single-photon sources in other $2 \mathrm{D}$ materials. Following that, NV centers were discovered in insulating h-BN flakes [80]. In the past three years, single-photon emitters have been identified in other TMDCs like $\mathrm{WS}_{2}$ [46], and narrow emission lines of localized centers have been found in $\mathrm{MoSe}_{2}$ [81, 82]. Recently, single photon emitters were also observed in GaSe flakes [83]. The origin of the quantum emission in atomically thin TMDCs has been related to strain-induced local confinement potentials, which trap free excitons $[84,85,86]$. In contrast to conventional semiconductors such as bulk silicon or GaAs, the extraordinary mechanical properties of 2D materials allows for applying strain of a few percent and strong nanoscale bending created by local imperfections in the 2D flatland. One of the primary result of this thesis is the discovery of these localized 0D exciton states in monolayer TMDC. We will not get into any further details here as chapter 3 will provide a more elaborate discussion on their optical properties based on our experimental results. Next, in chapters 
4-6 we will present devices exhibiting manipulation of these optical properties via external fields.

\subsection{Interlayer excitons}

Besides the excitons outlined in the previous sections 2.1-2.4, there is another type of exciton that is observed in the heterostructure of the $2 \mathrm{D}$ materials. Interlayer excitons in 2D materials were first studied in TMDCs. They are created by stacking two different materials forming a van der Waals heterostructure which was introduced in chapter 1 . The bands in these two materials align in such a way that the energy minimum of the conduction band lies in one layer while the energy maximum of the valence band lies in the other material forming a type II heterojunction. On optical excitation, an exciton is formed from the hole that is contributed by one material while electron contributed by the other material. This exciton recombines leading to the investigation of interlayer excitons. Their long lifetime makes them more suitable than the intra-layer excitons for certain applications. For example, interlayer valley excitons in 2D heterostructures may be a better system to explore valley manipulation. This is because the valley depolarization from electron-hole exchange interaction, that is proportional to the electron-hole overlap probability, is greatly reduced in an interlayer exciton owing to the electron-hole spatial separation [65]. However this type of exciton is introduced only for the sake of completeness of this chapter and is not one of the subject of study in this thesis. 


\subsection{Summary}

In this chapter, we have hiked along the different properties of excitons present in $2 \mathrm{D}$ to $0 \mathrm{D}$ to interlayer TMDCs. Their unique band structure and monolayer nature have resulted in strongly bound excitons and trions that can be probed through photoluminescence or reflectance measurements. These properties make the semiconducting cousins of graphene highly desirable in optoelectronics. Inversion symmetry breaking in the monolayer crystal lattice allows contrasted circular dichroism in different k-points, which takes the extreme form of optical selection rules for excitonic transitions at the different $\mathrm{K}$ valleys. This is analogous to spin dependent optical activities in semiconductors leading to the concept of valleytronics. Further, localized exciton states giving rise to single photon emitters have been introduced which will form the major part of discussion in this thesis in the later chapters. A brief description of interlayer excitons has also been presented. These observations pave the way for quantum valleytronics devices based on these exceptional materials. 


\section{Chapter 3}

\section{Quantum light emission from $2 \mathrm{~d}$ semiconductors}

In the previous chapter, we had introduced the different properties of excitons in $2 \mathrm{D}$ materials. The objective of this chapter is to investigate the effects of $3 \mathrm{D}$ quantum confinement in the excitons of $2 \mathrm{D}$ materials [74, 81]. Their quantum nature and various photo-physical properties are studied by employing photon counting and optical spectroscopic techniques.

\subsection{Introduction}

Single photon sources are essential for a number of emerging quantum information processing and quantum networking applications [87]. Of all the possible quantum emitters, epitaxially grown options are leading candidates for practical applications [88]. They are typically embedded in a bulk three-dimensional matrix that provides stability for a long time. Further their compatibility with established optoelectronic technologies enable heterostructure devices and monolithic photonic structures for enhanced performance and functions [89]. However, the bulk environment provides obstacles and limitations for engineering efficient light sources. 
Also, a high dielectric material poses a challenge to efficiently extract photons into a single optical mode [89]. Moreover, expensive hardware is required in reactive chemical techniques for manufacturing epitaxially grown dots. Currently, research is focused on identifying high optical quality single photon sources that are relatively simpler to produce. The classes of emitters in this genre includes colloidal quantum dots, organic single molecules or color centers in wide band gap semiconductors like color centers in diamonds, silicon carbide, etc. While the organic emitters rapidly photo-degrade, the color centers are not easily integrable into modern day scalable optoelectronic devices due to the complicated device fabrication it needs to go through before it can be actually used. So far, single photon emitters confined only in $1 \mathrm{~d}$ (eg carbon nanotubes, nanowires) or 3d structures have been studied in an overwhelming majority of quantum-optical experiments. However, 2D semiconductors can be an intriguing alternative to host a quantum emitter in the solid state which has been relatively unexplored.

Recently, several research groups including ours have discovered naturally occurring quantum dot-like emitters within atomically thin 2D materials like tungsten diselenide $\left(\mathrm{WSe}_{2}\right)[74,76,77,75,78]$, molybdenum diselenide $\left(\mathrm{MoSe}_{2}\right)[81,82]$ tungsten disulphide $\left(\mathrm{WS}_{2}\right)$ [46], hexagonal boron nitride (h-BN) [80] and gallium selenide [83]. The 2D nature of these material allows the most practical integration of single photon emitters into quantum devices due to their flexibility and ease of device assembly through van der Waals heterostructure. Besides, it eliminates the high index environment that hinders photon extraction making it fully compatible with integrated photonic approaches [90]. Moreover, using nanoscale strain engineering it is possible to generate a $2 \mathrm{~d}$ lattice of deterministically positioned high purity single photon emitters [86, 85, 91, 84]. In this chapter, optically active localized states in single layer $\mathrm{WSe}_{2}$ and $\mathrm{MoSe}_{2}$ has been introduced. Some 
of the photo-physical properties of these localized emitters are presented. Finally, the influence of external magnetic field and backgate voltage dependent emission is investigated.

\subsection{Sample preparation}
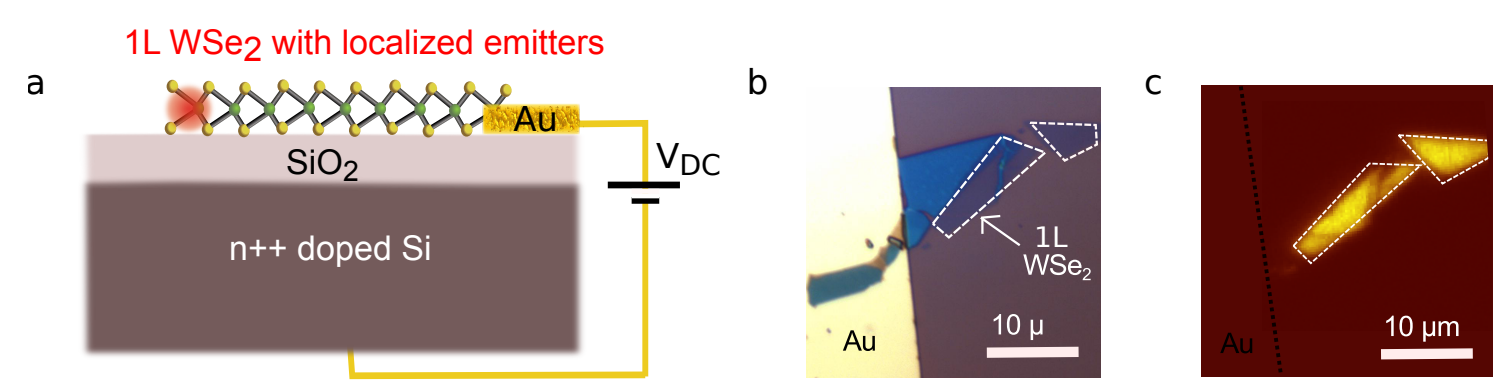

Figure 3.1: Schematic of the device: a. A single layer tungsten diselenide flake $\left(1 \mathrm{~L} \mathrm{WSe}_{2}\right)$ hosting localized emitters is transferred onto pre-patterned gold electrode on with doped silicon as backgate. b. Optical micrograph of the device. White dashed boxes outlines the monolayer region of the flake c. Room temperature scanning fluorescence map from the device. The single layer region shows maximum fluorescence counts (in yellow).

Owing to the weak interlayer van der Waal's forces a single layer flake can be isolated by micromechanical cleavage. We use scotch tape to exfoliate the monolayer from mineral bulk crystal on a PDMS based stamp using viscoelastic stamping technique [48]. The flake on the PDMS stamp is then carefully transferred onto the substrate. For voltage controlled studies, they are transferred on pre-patterned electrodes by careful alignment using a micro-manipulator stage. Details on the transfer technique is presented in section 1.5 of chapter 1 . The $\mathrm{MoSe}_{2}$ flake was transferred on a degenerately doped silicon substrate with 300 
nm of thermally grown oxide layer. The $\mathrm{WSe}_{2}$ sample was transferred onto photolithographically patterned electrodes consisting of $5 \mathrm{~nm}$ of chromium (Cr) and 40 $\mathrm{nm}$ gold $(\mathrm{Au})$ film deposited using electron-beam evaporation in a physical vapor deposition system. The substrate used was a degenerately n-doped silicon with a $300 \mathrm{~nm}$ thermally grown silicon dioxide $\left(\mathrm{SiO}_{2}\right)$ for exercising back-gate voltage control. A schematic of the back-gated device with gold electrodes is shown in Fig. 3.1a. The size of the exfoliated flakes are within $1000 \mu \mathrm{m}^{2}$. Monolayers are usually identified by contrast in the optical microscope [18] or from optical signatures in photoluminescence and/or Raman spectra (Refer to subsections 2.1.1 and 1.4.3). An exemplary optical micrograph of a TMDC flake on patterned electrode and its corresponding room temperature scanning fluorescence image for optical identification of the monolayer region is presented in Fig. 3.1b- 3.1c.

\subsection{Setup}

For low temperature optical spectroscopy, the device is mounted on a three-axis piezo-stage in a liquid Helium (He) cryostat $(4.2 \mathrm{~K})$ as shown in the schematic of Fig. 3.2 and Fig. 3.3c. The sample is connected to a Keithley 2400 source-meter through electrical breakouts for applying electrical bias. It is optically excited by focussing a green diode laser $(\lambda=532 \mathrm{~nm})$. The laser is collimated after a single mode fiber input using an aspheric lens and is then focussed at the sample plane by an Attocube objective lens of numerical aperture (NA) of 0.82 . This lets us sample a diffraction limited spot-size [92], $d=\lambda / 2 N A$. The luminescence is collected by the same objective lens and separated from the excitation beam using a beamsplitter (B, 92:8). The collected light passes through a $550 \mathrm{~nm}$ long pass filter (LPF) where the excitation source is filtered from the sample fluorescence 


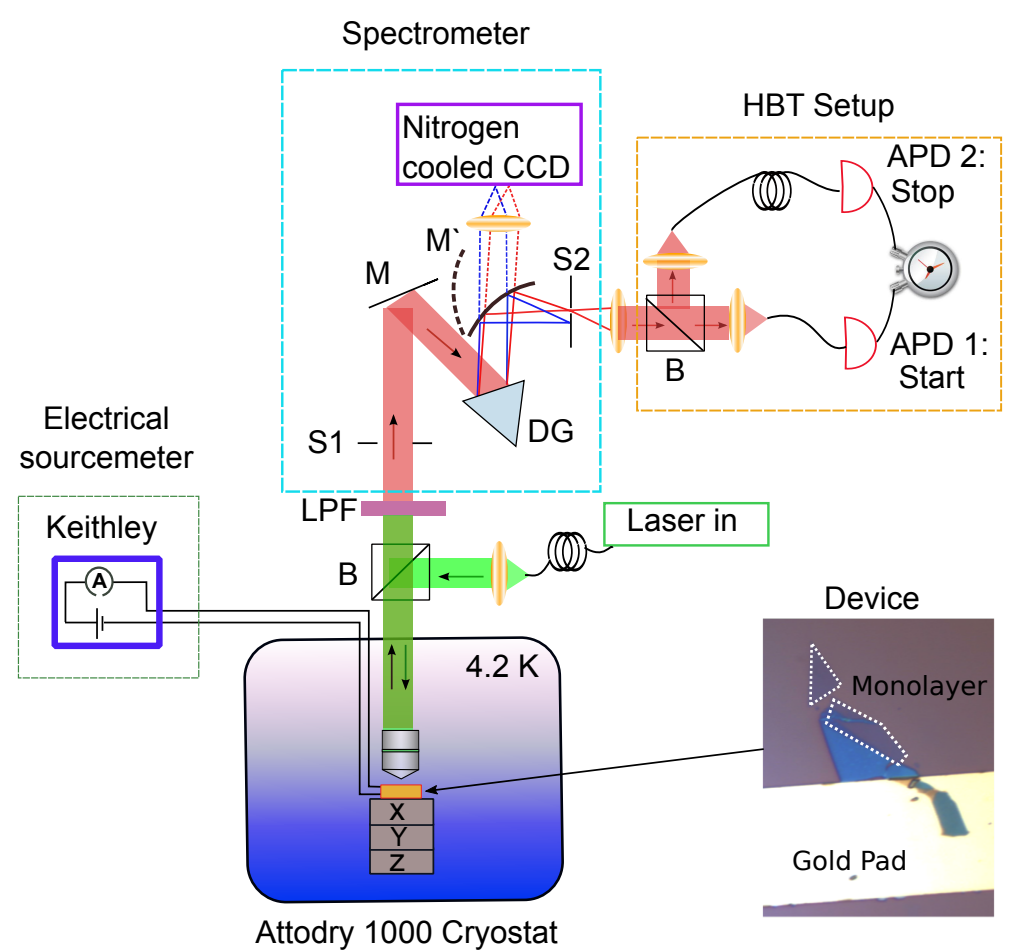

Figure 3.2: Low temperature magneto-optical setup: The system has an additional module to measurement intensity autocorrelations via the Hanbury Brown and Twiss (HBT) technique.

and the signal is then focussed at the input of the single mode fiber (Thorlabs P3-630A-FC) at the collection port. In our setup, the conventional pinholes for confocal microscopy is replaced by single mode fibers which allows us to perform micro-photoluminescence spectroscopy. The signal is then guided to the entrance slit (S1) of an imaging spectrometer ( Acton Spectrapro, Model 2750i) with liquid nitrogen cooled silicon charge-coupled device (Si- CCD, Princeton instruments) camera for measuring the spectral information from the flakes. The spectrometer has a triple grating which ultimately provides resolutions of $22 \mu \mathrm{eV}(1800 \mathrm{l} / \mathrm{mm})$, $40 \mu \mathrm{eV}(1200 \mathrm{l} / \mathrm{mm})$ and $400 \mu \mathrm{eV}$ (300 l/mm) in optical spectroscopy. 


\subsubsection{Intensity autocorrelation setup}

In the setup described above, the luminescence can also be directed towards a second exit slit (S2) by a flip mirror $\left(\mathrm{M}^{\prime}\right)$ to a pair of photon counters for studying photon statistics through second order correlation measurements. The signal collected from a focal spot on our sample usually consists of multiple emitters that are spectrally separated from each other. Therefore, we use a combination of the grating and exit slit in our spectrometer to spectrally filter a single emission peak. Firstly, the grating in the spectrometer disperses the signal from multiple emitters. The grating is centered on a particular emitter and this is directed towards an exit slit which spatially filters the dispersed light such that the collected light consists of signal from a single quantum emitter. Then, the signal is split into two using a 50:50 beamsplitter and the output ports are connected to two avalanche photo-diodes (APD) corresponding to the start and stop channel as indicated in Fig. 3.2. A counter records the time interval between two consecutive photon detection event at the two detectors producing a histogram of coincidence counts.

\subsubsection{Polarization resolved setup}

Polarization control and measurement is one of the key interest for the study of the localized and delocalized excitons in TMDC. As we use a common objective for excitation and collection, our input and output signal overlap each other. This is separated by a beamsplitter as shown in Fig. 3.3. However, this beamsplitter has different reflection and transmission coefficient for light polarized parallel (p) or perpendicular (s) to the reflection plane of the beamsplitter. Now, for the case of a linearly polarized light, this problem can be easily solved by orienting the polarization along s or p direction, so the contribution from the other component is minimized. However, circularly polarized light will be rendered elliptically 

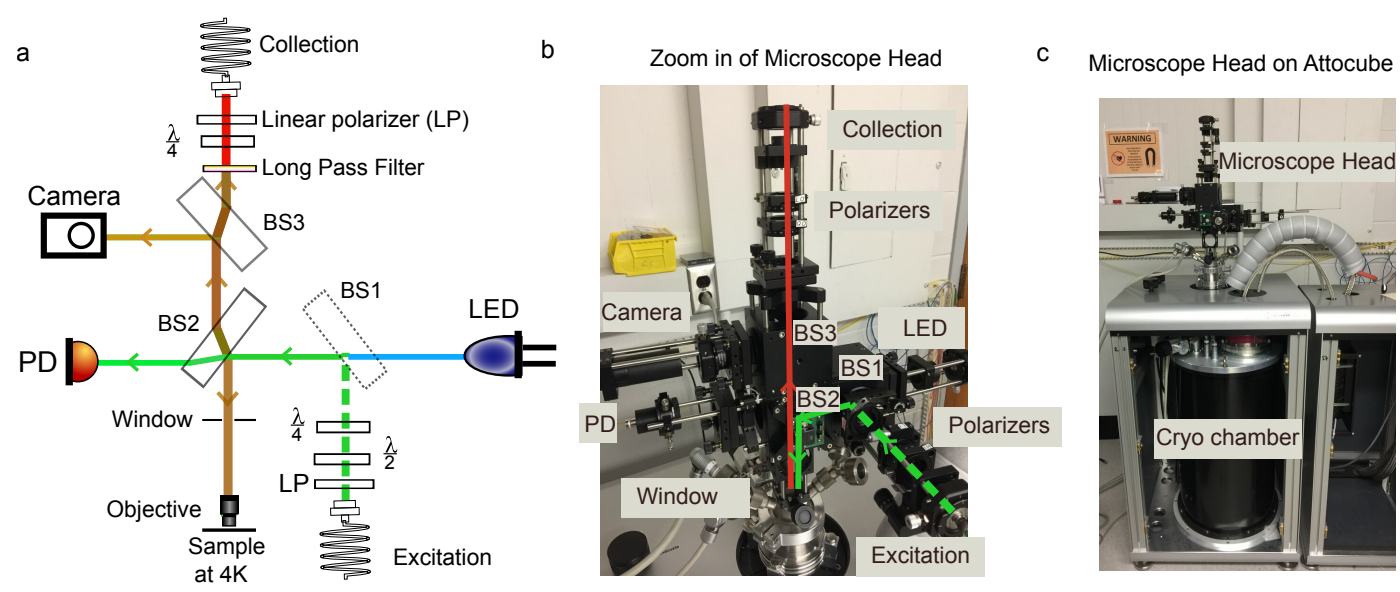

Figure 3.3: Polarization resolved setup: a. Schematic of the polarization resolved microscope head b. Snapshot of the actual microscope head c. Microscope head integrated with the Attocube cryostat chamber.

polarized due to this anisotropy of the reflection coefficient for the s and p components. An elegant solution to this problem is using a second beamsplitter (BS1), oriented orthogonal to the plane of light propagation axis with respect to the first beam splitter (BS2) (Fig. 3.3a-b) so the s and p components are inverted between the two beamsplitters. A schematic and snapshot of the actual microscope is presented in Fig 3.3. Laser is coupled through an optical fiber intro the excitation arm where it reflects at NBK7 broadband precision window acting as beamsplitters, BS1 and BS2 respectively. Indeed, if the two beamsplitters are perfectly identical their effective s and p reflection coefficients will be the same. If we orient them in order to reflect the incoming light into perpendicular directions, the axis perpendicular to the reflection plane (s) for the first beamsplitter will be the axis parallel to reflection plane for the other $(\mathrm{p})$. So, the transmitted light will be attenuated by a factor of the reflection coefficient of the $\mathrm{s}$ and $\mathrm{p}$ components but its polarization will be unchanged. We use achromatic quarter wave plate $(\lambda / 4)$, achromatic half wave plate $(\lambda / 2)$ and linear polarizers (LP) to prepare or 
analyze circularly or linearly polarized light as required by the experiments. For the collection path, an additional beamsplitter, BS3 is added to correct for the displacement in the path due to refraction through the BS2. Additionally, it offers an extra port for wide-field imaging of the sample via LED excitation as shown in the schematic and the snapshot (Fig 3.3a,b). The setup lets us prepare and detect circularly polarized light with a contrast of $99.8 \%$ and $98.6 \%$ respectively.

\subsection{Optical identification: Micro-Photoluminescence}

\section{spectroscopy}
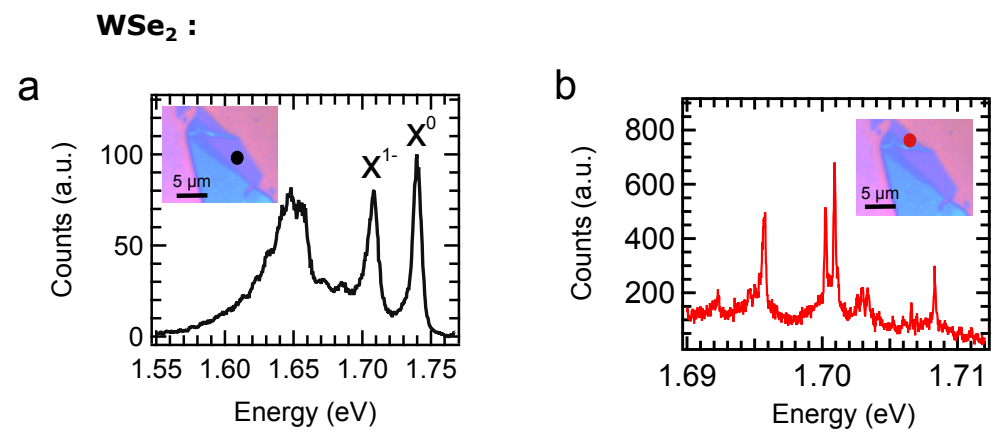

$\mathrm{MoSe}_{2}$ :

C

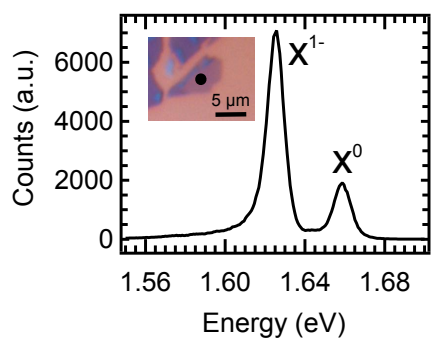

d

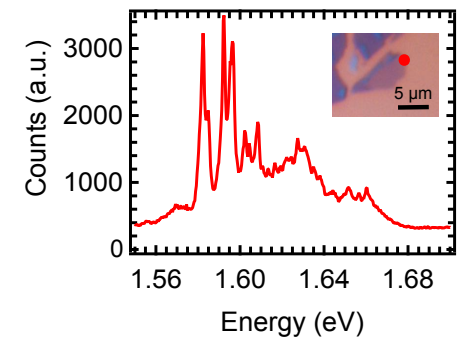

Figure 3.4: PL identification: a,c PL spectrum of delocalized excitons from a region on the monolayer $\mathrm{WSe}_{2}$ and $\mathrm{MoSe}_{2}$ represented by black dot on the optical image of the inset $\mathbf{b}, \mathbf{d}$ Localized emission from quantum dot-like emitters from certain region near the edge of the flake (red dot marked on inset). 
We perform micro-photoluminescence spectroscopy using the setup described in section 3.2 for identification of localized and delocalized exciton species in the TMDCs. Figure 3.4a and c presents the low temperature (4K) PL spectrum measured from pristine monolayers of $\mathrm{WSe}_{2}$ and $\mathrm{MoSe}_{2}$ flakes respectively. For both the materials, we observed peaks corresponding to neutral exciton $\left(\mathrm{X}^{0}\right)$ and trion, which, in this case is a negatively charged exciton $\left(\mathrm{X}^{1-}\right)$. In addition to this exciton emission, there is a strong broadband emission from $\mathrm{WSe}_{2}$ [Fig. 3.4a] within 1.6-1.7 eV which is attributed to impurities in crystal [93]. However, at some regions near the edge, the interface of a single and multilayer region, or any crystal imperfections (like cracks, folds or wrinkles in the 2D monolayer), the characteristics of the PL emission changed drastically for both the flakes as shown in Fig. 3.4b and d. The PL spectrum at these positions show distinctly narrow lines (linewidth less than 3 orders of magnitude from the delocalized excitons) which are red shifted from the delocalized excitons. The linewidth of the localized emitters ranges from $100 \mu \mathrm{eV}-500 \mu \mathrm{eV}$ while the delocalized exciton is within $4 \mathrm{meV}-7 \mathrm{meV}$ depending on sample quality. The peak energy of the $\mathrm{WSe}_{2}$ delocalized exciton (trion) is around $1.74 \mathrm{eV}(1.71)$, while the localized excitons ranges anywhere between $1.55 \mathrm{eV}-1.71 \mathrm{eV}$. Similarly, for $\mathrm{MoSe}_{2}$, the exciton (trion) emission energy is around $1.66 \mathrm{eV}(1.625 \mathrm{eV})$, and the localized emitters are observed mostly within $1.56 \mathrm{eV}-1.64 \mathrm{eV}$.

To study the simultaneous spectral and spatial localization of these emitters, we perform 1D hyper-spectral imaging as presented in Fig. 3.5a. One-dimensional line cuts extracted from Fig. 3.5a for two distinct spectral lines $(1.668 \mathrm{eV}$ and $1.716 \mathrm{eV}$ ) are shown in Fig. 3.5b. The inset in 3.5b identifies the path for the hyper-spectral line cuts. The start and stop positions are indicated by arrows. Interestingly, we observe these features along the interface of the single and mul- 

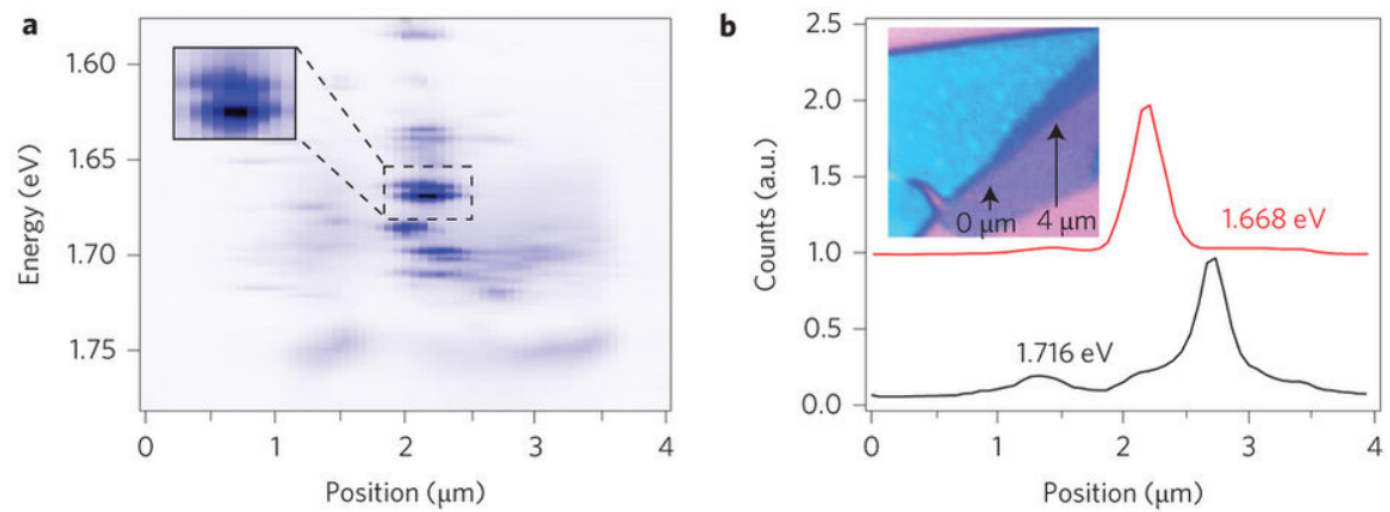

Figure 3.5: Hyperspectral data: a. One-dimensional hyperspectral image of $\mathrm{WSe}_{2}$ quantum emitter exhibiting spatial localization. Inset: Zoom-in of the black dashed box in the main image. b. One-dimensional line cuts from two different quantum emitters. Inset: Optical microscope image of the flake. The start and finish locations of the line cut are identified by two arrows. Total line cut, $4 \mu m$. Figure adapted from [74] with permission.

tilayer flakes (data shown in Fig. 3.5a) and along the edges of flakes. The typical spatial localization of these narrow features is of the order of the diffraction limited resolution from our confocal microscope setup.

\subsubsection{Power dependence}

Next, we present some photo-physical properties of these emitters. A comparative study of the power dependence from the localized excitons with respect to the delocalized excitons in presented in Fig. 3.6. As exhibited in Fig. 3.6a the delocalized neutral exciton (open diamonds) and trion (open squares) in $\mathrm{MoSe}_{2}$ show a linear dependence as a function of power without any sign of saturation. However, increasing the optical power results in non-linear dependence of the PL intensity suggestive of emission from localized or defect bound states [93]. In Fig. 
a

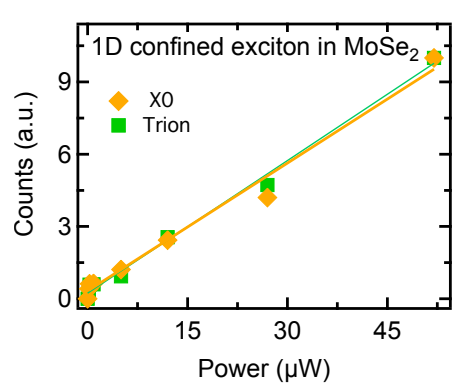

b

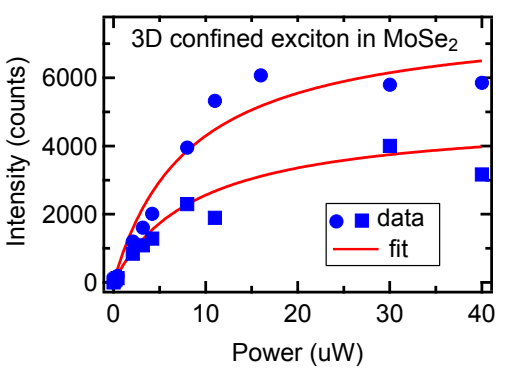

C

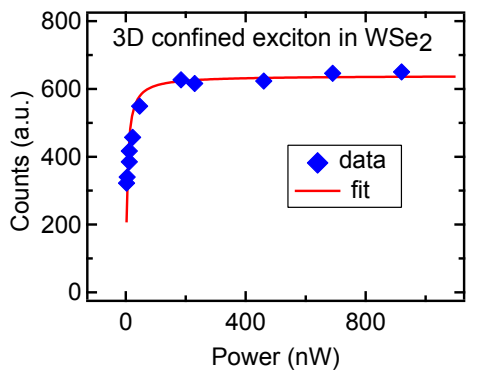

Figure 3.6: Power dependence: a. Integrated PL intensity from delocalized exciton emission from trion (green square) and neutral exciton (X0) (orange diamonds) as a function of incident power showing linear response to the incident power. b. Power dependence for two different localized emitters in $\mathrm{MoSe}_{2}$ showing saturation at different powers. c. Power dependence from a quantum emitter in $\mathrm{WSe}_{2}$. Data from b-c are fit to power saturation curve. Figure adapted from [74] with permission.

$3.6 \mathrm{~b}$ and $\mathrm{c}$, we plot the power dependence of emission from two different emitters in $\mathrm{MoSe}_{2}$. The power saturation curve [74, 80, 85, 94]

$$
I=I_{\text {sat }} \frac{P}{\left(P_{\text {sat }}+P\right)}
$$

is used to fit the data, where, $\mathrm{I}_{\text {sat }}$ and $\mathrm{P}_{\text {sat }}$ are the value of counts and power at saturation respectively. The localized emitters have different saturation power $\left(\mathrm{P}_{\text {sat }}=8.2 \mu \mathrm{W}\right.$ and $9.1 \mu \mathrm{W}$ for D1 (blue circle) and D2 (blue square) respectively), but they both show saturation after a certain optical power level. Similarly, delocalized exciton in $\mathrm{WSe}_{2}$ exhibit linear power dependence [75]. However, for the exemplary localized emitter in Fig 3.6c, the emission saturates at $50 \mathrm{nW}$ input power. This kind of behavior was previously observed for excitons that are bound to defect sites at the flake [93]. At low power only a small fraction of the free electron and hole pair form exciton. On increasing the excitation 
power, the localized states are populated with more excitons per unit time which is limited by the lifetime by the emitter, thus, saturating at higher powers. This is responsible for the nonlinear dependence of PL intensity with excitation power for the localized or defect bound excitons, whereas for the free excitons (neutral exciton and trion), the intensity increases linearly with power. Power dependence studies have been used to identify direct recombination process of free excitons from defect mediated transitions in quantum wells [95] as well as defects in TMDCs $[74,76,77,75,78,93]$. Every localized emitter studied in these experiments exhibit saturation after a certain power level which is a signature of localized emission.

\subsubsection{Temperature dependence}

a

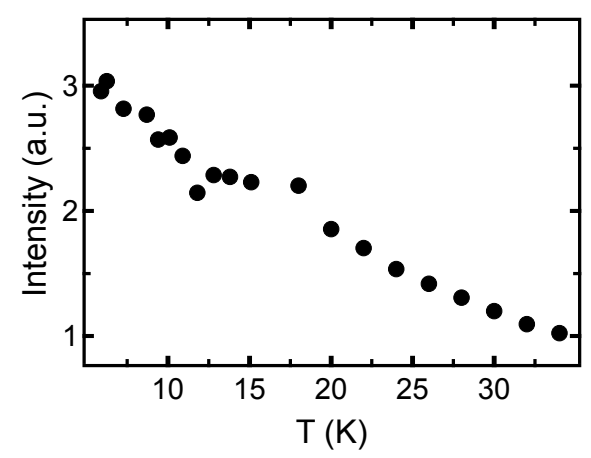

b

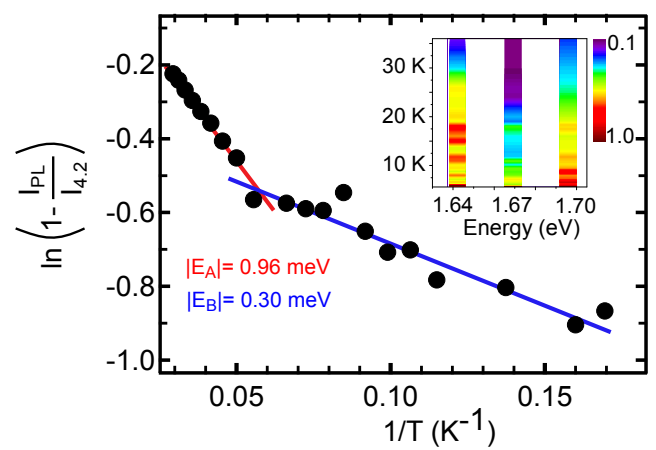

Figure 3.7: Temperature dependence: a. Temperature dependence of a localized emitter in $\mathrm{WSe}_{2}$, b. Arrhenius plot for data in a, showing two different slopes. Inset: Temperature $(\mathrm{T})$ dependence of multiple quantum dots. The bottom of the panel corresponds to $4.2 \mathrm{~K}$ and top to $34 \mathrm{~K}$. The color bar is normalized such that the maximum number of photocounts is set to 1 . Figure adapted from [74] with permission.

Fig. 3.7a presents the temperature dependent PL intensity from the localized 
emitters. The PL emission is nearly suppressed above a certain temperature. Fig. 3.7b presents the fit to the Arrhenius equation [96],

$$
I_{P L}=I_{4.2}\left(1-e^{\frac{-E_{a}}{k T}}\right)
$$

for the data in Fig. 3.7a, where $I_{4.2}$ is the low-temperature PL intensity and $E_{a}$ is the activation energy of the loss channel. As is apparent in the data, there is a change in slope at $\sim 18 \mathrm{~K}$. From the fit below $18 \mathrm{~K}$ we estimate an activation energy of $0.3 \mathrm{meV}$ and from the fit above $18 \mathrm{~K}, 0.96 \mathrm{meV}$. This decrease in PL intensity is attributed to thermal activation of a near lying dark exciton state [97]. We further find that the photoluminescence is restored when the temperature is lowered back to $4 \mathrm{~K}$, and is robust against multiple temperature cycling. Reduction of photoluminescence intensity with increasing temperature is also observed for the case of localized emitters in $\mathrm{MoSe}_{2}$ [81].

\subsubsection{Spectral Wandering}

The implementation of single photon sources in viable devices critically depend on the stability of the photon emission. There have been reports of colloidal quantum dots with diameters of few nanometers that typically suffer from blinking, bleaching and spectral diffusion. These effects are linked to the large surface to volume ratio and presence of surface defects and charges. For atomically thin TMDCs, the surface to volume ratios are even higher owing to their dimensionality. However, we have not observed photobleaching of the emitters for hours. A typical time trace of a $\mathrm{WSe}_{2}$ emitter exhibiting a jitter in the emission energy is presented in Fig 3.8a. For this particular emitter, PL spectra collected for a second amounts to a linewidth of $0.15 \mathrm{meV}$ (blue data in Fig 3.8b). When the PL spectra from the same emitter is integrated for a longer period of time (90 s integration), the 

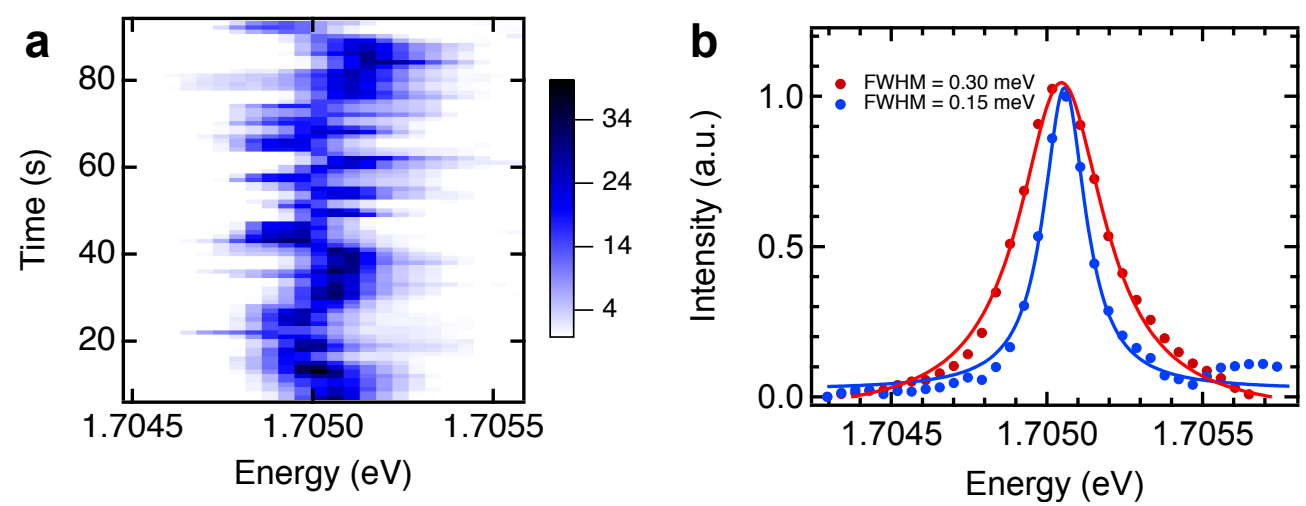

Figure 3.8: Spectral wandering: a. Time dependent PL trace showing spectral jitter of a quantum emitter within a $\sim 1 \mathrm{meV}$ spectral window. Color scale represents PL intensity in arbitrary units, b. Line cut at 1s integration time (blue data points). Red data is integrated PL intensity for $90 \mathrm{~s}$ duration calculated from a. The broadening of the FWHM of the integrated spectra represents the inhomogeneous broadening due to spectral wandering.

linewidth broadens by a factor of 2 due to spectral wandering (red data in Fig $3.8 \mathrm{~b})$. The inhomogeneous broadening due to spectral wandering ranges from 0.1 $\mathrm{meV}-0.5 \mathrm{meV}$ for the different studied emitters. This can be further reduced by near resonant excitation and transferring them to cleaner substrates like boron nitride [78] or epitaxially grown InGaP [98].

\subsection{Photon statistics}

All the above results compelled us to investigate the photon statistics to find out if these localized emitters are in fact a source of single photons. For this, we test for photon antibunching by measuring the second order intensity autocorrelation function $\left(g^{2}(\tau)\right)$. The second order correlation function is one of the most relevant 


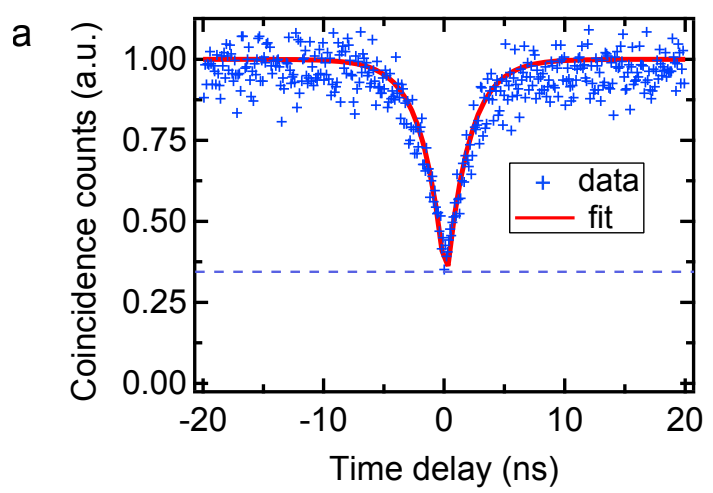

Figure 3.9: Photon antibunching: Second order autocorrelation function measured for a single emitter as a function of time delay using continuous wave laser excitation. Figure adapted from [74] with permission.

function for characterizing a light source. One major attribute is to distinguish non-classical antibunched light source from classical ones like thermal source. To measure this function, we make use of the Hanbury Brown and Twiss (HBT) interferometer [99] whose simplified schematic is shown in Fig. 3.2 as part of the setup. The first direct measurement of antibunching was performed here on this campus about four decades ago by Kimble et al while investigating the photon statistics in the fluorescence of a sodium atom beam [100].

In our setup (Fig. 3.2), the signal from a region on the sample with localized emitter is collected and sent to a spectrometer. The signal goes through the grating where it is split into different spectral components. A single peak corresponding to a single localized emitter is selected out of the comb-like spectrum as presented in Fig. $3.4 \mathrm{~b}$ and it is spatially filtered using the exit slit of the spectrometer. From there the signal is directed to the HBT interferometer where it is first split by a 50/50 beamsplitter onto two avalanche photodiodes (APDs). The output signal from the APDs are then connected to the start and stop channel of a time correlated photon counter from Ortec. The counter repeat- 
edly measures the time interval between the the clicks at the start and stop APD channels originating from two emitted photons that will be normally separated by a certain amount of time for the case of antibunched photons. A histogram of the co-incident counts as a function of the time delay is then generated. This measures the probability distribution of photon pair with time interval $\tau$. Furthermore, the stop channel is also delayed by a certain amount of time in order to visualize the negative correlations.

The measured correlation counts against the inter-photon time for an emitter in $\mathrm{WSe}_{2}$ is presented in Fig 3.9. A promising dip at zero time delay suggests that the emitters are indeed sources of single photons. The antibunching dip of the correlation function is based on the fact that a single emitter can only emit one photon at a time. One can easily describe this process using a two-level system having a lower energy ground state and higher excited state. A single emitter in an excited state requires a finite amount of time before it relaxes back to the ground state by emitting a photon. The temporal separation between adjacent photons is therefore determined mostly by the excited-state lifetime. This effect is known as antibunching and represents the sub-Poissonian nature of the emitted light. The experimental data is compared to an equation stemming from a two-level model [101]:

$$
g^{2}(t)=1-A e^{-\left|\frac{t}{\tau}\right|}
$$

A $g^{2}(t=0)$ value of 0.37 is obtained from the fit which is less than 0.5 confirming the antibunched nature of the photons. From the pronounced anti-bunching dip, the excited-state lifetime $(\tau)$ is determined to be 1.8 ns. Compared to extended $\mathrm{WSe}_{2}$ excitons, which have lifetimes on the order of $\sim 30$ ps [102], the marked increase in excited-state lifetime is characteristic of three-dimensional quantum confinement [103]. 
We do not observe any antibunching from from the localized emitters in $\mathrm{MoSe}_{2}$. This could be due to the presence of more than one emitter within the collected signal bandwidth or potentially short anti-bunching timescales compared to the $\sim 400$ ps instrument response time of our detection system.

\subsection{Dipole orientation}

a

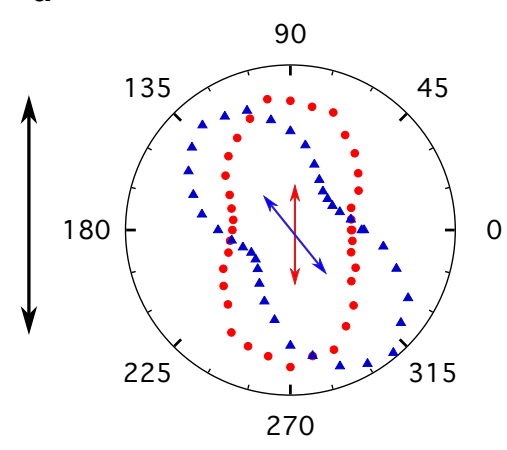

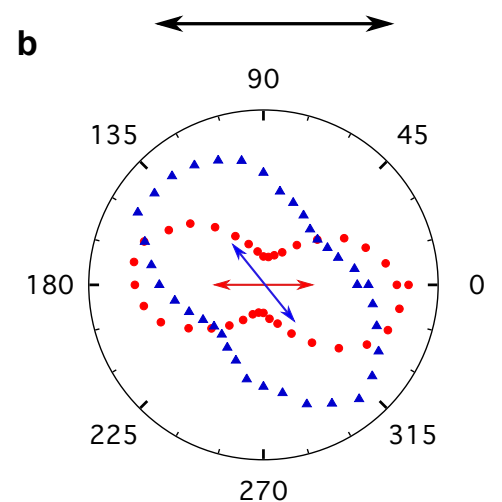

Figure 3.10: Dipole orientation: Polar plot of intensity vs emission angle from a quantum emitter (blue) vs 2D delocalized exciton (red) for a. vertical, and b. horizontal polarization of incident laser. The linear polarization of the excitation laser is indicated by black double arrow for the two polar plots.

It is possible to obtain information regarding the orientation of the dipole mediating the light emission by examining the emitted photon's polarization properties. Figure 3.10 indicates a preferred orientation of the polarization of a quantum emitter exciton and the delocalized exciton in $\mathrm{WSe}_{2}$ for the two orthogonal orientation of the linearly polarized input laser as indicated by black double arrows. The PL emission from the delocalized neutral excitons (red data) are polarized along the same sense of the excitation laser which is consistent with literature [67]. However, the emission from single localized emitter is linearly polarized (blue data 
points) along a certain direction irrespective of the polarization of input laser. The angular dependence of PL suggests that the emitters have an in-plane dipole moment just as the neutral exciton of delocalized excitons in TMDC, but unlike the exciton the dipole orientation is not determined by the direction of linear polarization of the excitation source. This evidence is also consistent with the observation that localized excitons are responsible for this emission since localized excitons are expected to have preferred orientation for their dipole moment. This preferred orientation of the dipole randomly varies from emitter to emitter, independent of the direction of the excitation source's polarization. This might be caused by anisotropic strain acting on the emitter, similar to other solid-state quantum dots [104].

\subsection{Magnetic field dependence}

Now that we have established that the sharp emission peaks spawn from optically active quantum dot-like emitters, we focus on studying some of the atomlike properties of the quantum emitters in $\mathrm{WSe}_{2}$ and $\mathrm{MoSe}_{2}$, like Zeeman effect, which is very commonly demonstrated by other solid-state quantum emitters. Furthermore, we also demonstrate polarization resolved magneto-optical PL. These measurements also help to determine if these emitters inherit the unique valley properties of their monolayer host.

\subsubsection{Fine structure splitting}

For the emitters in $\mathrm{WSe}_{2}$, at zero magnetic field, many quantum dots revealed a characteristic doublet peak with a splitting $\Delta_{0}$, as seen in the spectra of Fig 3.11a. The two split peaks also demonstrate a correlated spectral wandering which 

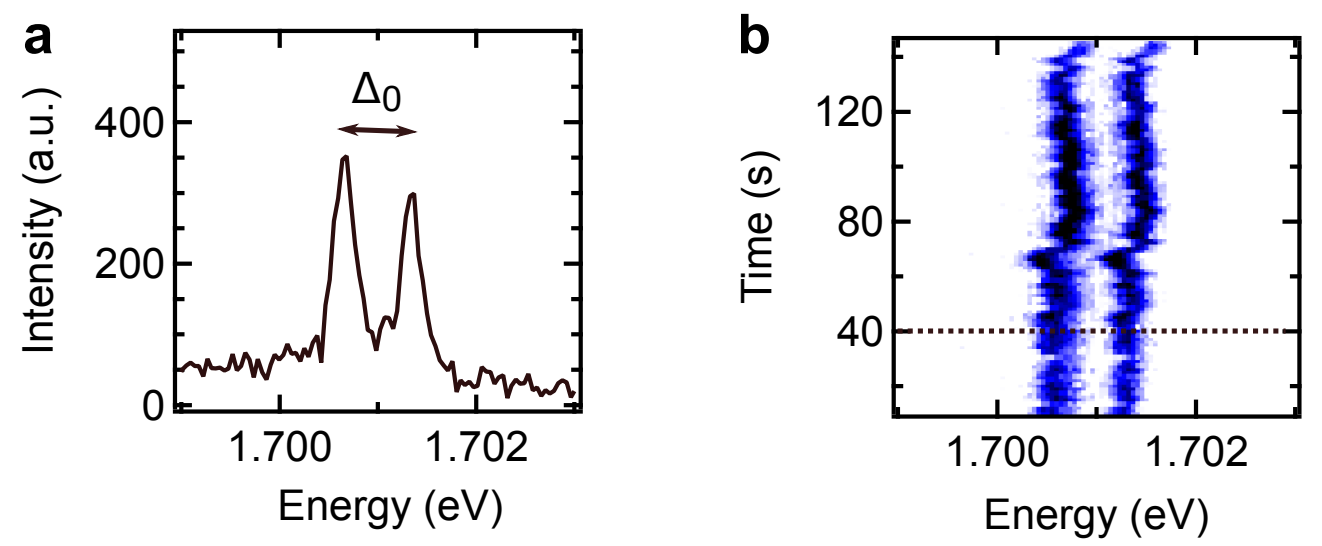

Figure 3.11: Fine structure splitting: a. Emission spectra from characteristic doublet peaks b. Correlated spectral jitter in the time trace of the doublet. The horizontal dotted line represents the position of the spectral line-cut in a.

suggests that the peaks originate from the same emitter (Fig. 3.11b). It has well established in InAs quantum dots that an anisotropic exchange interaction can split the emission of the bright neutral exciton states into two spin-mixed, linearly polarized exciton states, commonly referred to as a fine-structure-splitting (FSS) [105]. In the following sections, we aim to demonstrate that the fine structure splitting in the $\mathrm{WSe}_{2}$ quantum emitters is possibly due to very similar inter-valley electron-hole exchange interaction in these confined states.

\subsubsection{Zeeman effect in $\mathrm{WSe}_{2} \mathrm{QD}$}

In this section, the interaction of an external magnetic field (Faraday geometry) with the atom-like electronic states of the quantum dots are measured via the magneto-optical PL measurements. These measurements reveal if the doublet structure is related to similar spin mixed states as the epitaxially grown QDs. In Fig. 3.12a, it is seen that the doublet's peak separation increases as a function of magnetic field which is attributed to Zeeman splitting. The peak energies are 

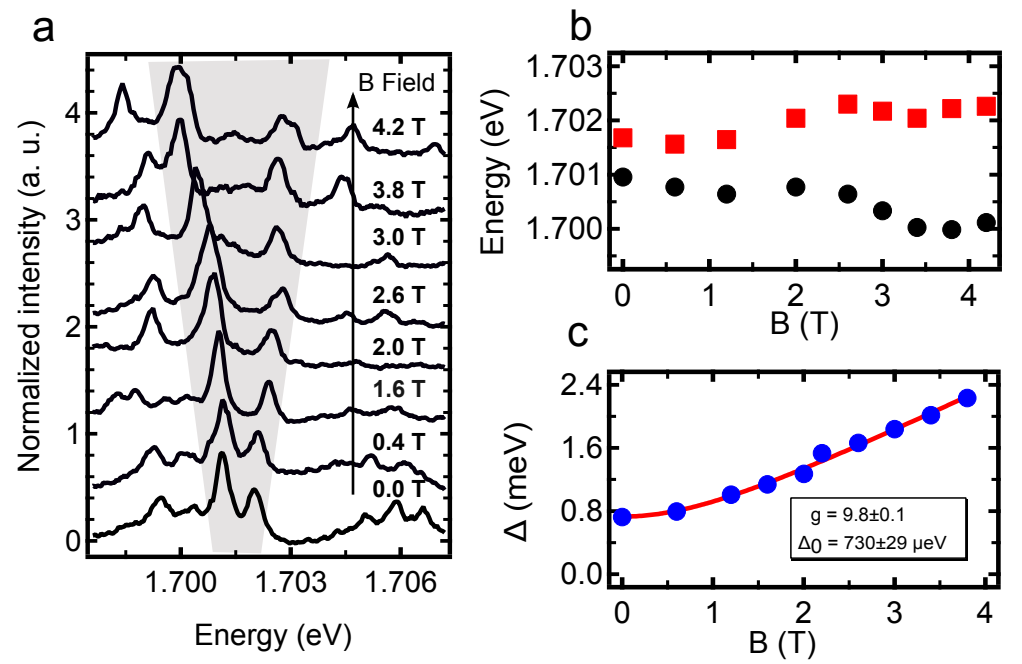

Figure 3.12: Magneto-optical studies of the localized emitters in $\mathrm{WSe}_{2}$ : a. Magnetic field dependence of a QD emission spectra in the Faraday configuration. The grey shading identifies the splitting as a function of applied B field. b. Spectral location of two emission peaks. c. Spectral separation of QD emission peaks (blue circles) as a function of magnetic field fitted with a hyperbolic function (red curve) to extract the g-factor and zero field splitting. Figure adapted from [74] with permission.

extracted in Fig. 3.12b and the relative separation of the splitting is plotted in 3.12c. The data is fit to the equation:

$$
\triangle=\left(\sqrt{\left.\left(g \mu_{b}\right)^{2}+\triangle_{0}^{2}\right)}\right.
$$

where, $\mathrm{g}$ is the exciton g-factor, $\mu$ is the Bohr magneton, and $\triangle_{0}$ is the zero-field splitting. From the fit a g-factor of 9.8 is extracted which is nearly an order of magnitude larger than the delocalized valley excitons in TMDC [71] or in InAs quantum dots [105]. For the different measured emitters, the g-factors ranges from 7-10. The reason for the large g-factor is not yet determined. However, these range of values are consistent with results from other groups [76, 77, 75]. that were published in parallel with our results [74]. The zero-field splitting is 
measured to be about $700 \mu \mathrm{ev}$ for this particular emitter. The values of the zero field splitting ranges from $400 \mu \mathrm{ev}-800 \mu \mathrm{ev}$. This is also larger than the epitaxially grown QDs consistent with the strong coulomb interactions in TMDCs.
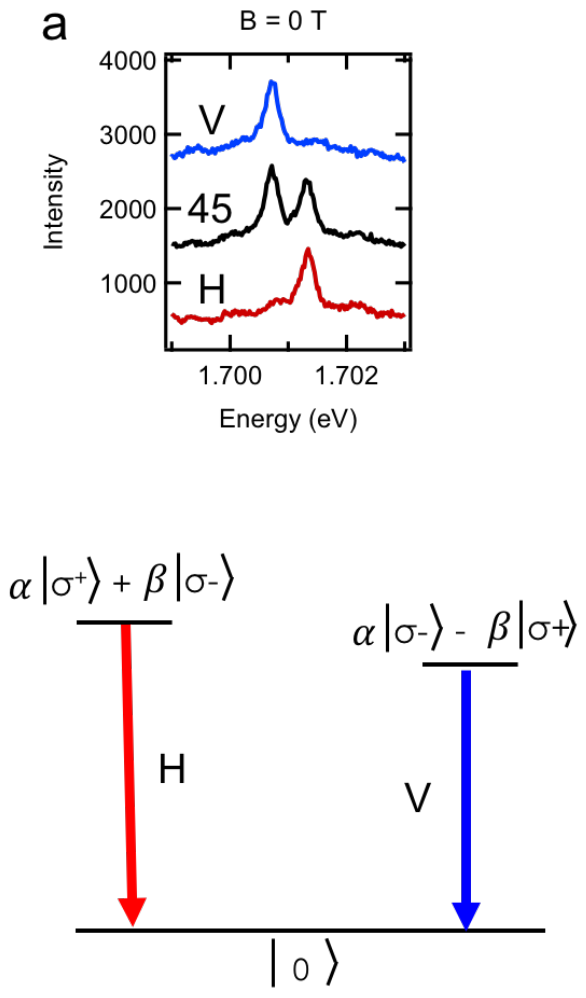
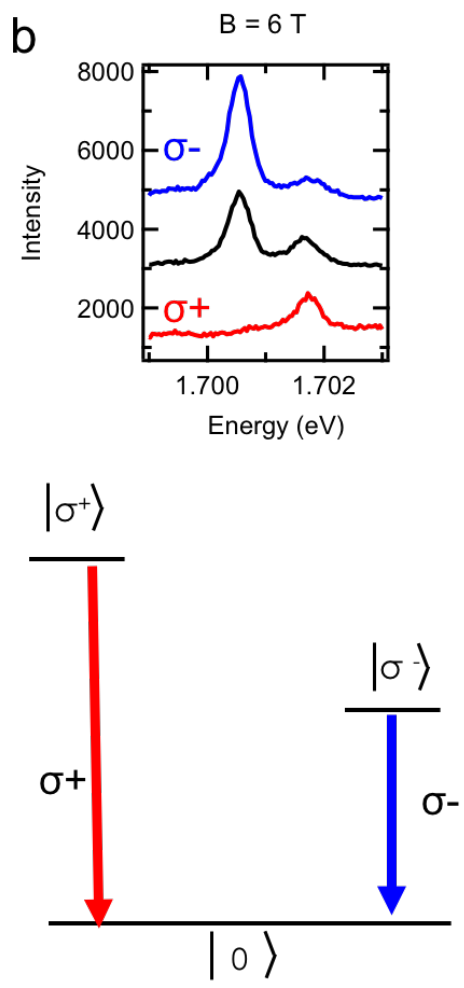

Figure 3.13: Polarization vs B field: a. Cross linearly polarized emission at B $=0 \mathrm{~T}$ b. Cross circularly polarized doublets at $\mathrm{B}=6 \mathrm{~T}$. The bottom panel is a representation of the two states of the doublet that are linearly (circularly, $\sigma)$ polarized at $\mathrm{B}=0 \mathrm{~T}(6 \mathrm{~T}) .\left(\mathrm{H}=\right.$ horizontal, $\mathrm{V}=$ vertical, $45=45^{0}$ linear polarization)

From polarization resolved studies in Fig 3.13a, we have found that the peaks of the doublet are cross linearly polarized at zero magnetic field. However, on applying a magnetic field (Fig 3.13b), the peaks of the doublet become cross circularly polarized. The doublets emission polarization remarkably resembles the 
spin mixed neutral exciton states in the epitaxially grown InAs QDs [105]. For the case of shallow confinements in TMDCs, it has been predicted that the bound states of these localized emitters will be formed predominantly from the band edge Bloch states in the $\mathrm{K}$ and $-\mathrm{K}$ valley of the $2 \mathrm{~d}$ TMDC [106]. Due to intervalley coupling introduced by the confinement potential, the bloch states from the $\mathrm{K}$ and -K valleys are hybridized. Now, if there is an anisotropy in the confinement, the anisotropic intervalley electron-hole exchange interaction hybridizes the valleys and splits the exciton. The two new eigen states of the neutral exciton can be represented by $\alpha\left|\sigma^{+}\right\rangle+\beta\left|\sigma^{-}\right\rangle$and $\alpha\left|\sigma^{-}\right\rangle-\beta\left|\sigma^{+}\right\rangle$where $|\alpha\rangle$ and $|\beta\rangle$ are coefficients related to the degree of valley hybridization (Bottom of Fig 3.13a). However, on application of a magnetic field in the Faraday configuration, the Zeeman energy overcomes the exchange energy resulting in the recovery of the circularly polarized $\left|\sigma^{+}\right\rangle$and $\left|\sigma^{-}\right\rangle$states.

\subsubsection{Zeeman effect in $\mathrm{MoSe}_{2} \mathrm{QD}$}

Figure 3.14a shows the PL spectra of the localized emitters in $\mathrm{MoSe}_{2}$ at varying magnetic fields. D1 and D2 represent two emitters showing a clear Zeeman splitting as the magnetic field is gradually ramped up. At zero field, no measurable fine structure splitting could be observed. Any fine structure splitting in the $\mathrm{MoSe}_{2}$ localized emitters, if present, could be smeared out either due to spectral diffusion and/or resolution of the spectrometer in our current setup. We extract the peak energies for the splitting as a function of magnetic field and plot these in Fig. 3.14c. The split peaks also wander together which is apparent in 3.14b. The Zeeman splitting $(\Delta)$ as a function of the magnetic field can be fit to the linear equation for the case of $\mathrm{MoSe}_{2}$ :

$$
\Delta=g \mu_{B} B
$$



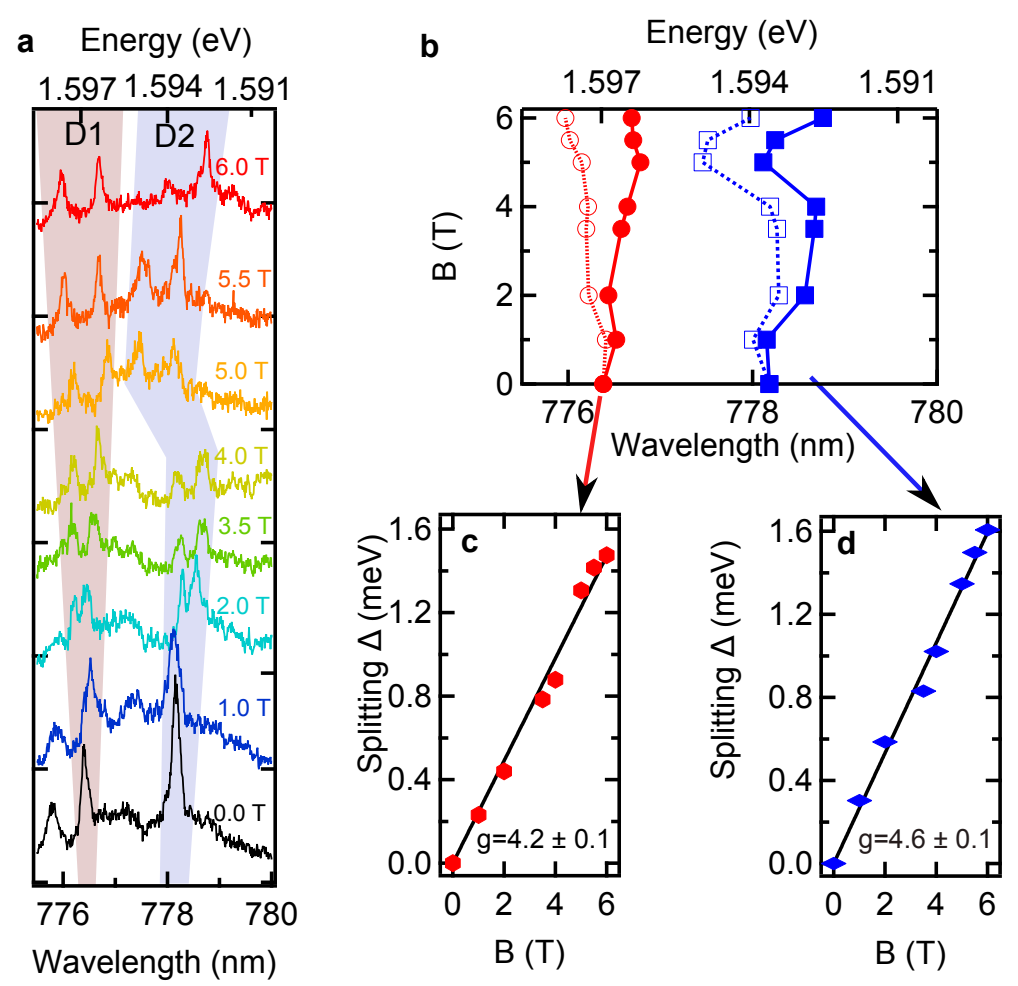

Figure 3.14: Magneto-optical studies of the localized emitters in $\mathrm{MoSe}_{2}$ : a. Zeeman splitting of two emission lines as a function of magnetic field. The red and blue shaded region are guides to eye showing the trail of the two localized emitter peaks D1 and D2. b. Extracted peak energies of D1 and D2 from Fig. 3.14a showing the similar spectral wandering from the split doublets. c, d. The splitting energy from the two doublets are calculated and fit from where the $\mathrm{g}$ factors are calculated to be around 4.6 and 4.2 respectively. The resolution of the grating is within by the size of the marker used.

where $\mathrm{g}$ is the g-factor, $\mu_{B}$ is the Bohr magneton, and $\mathrm{B}$ is the applied magnetic field. From the fit, we find the g-factor to be 4.2 and 4.6 respectively for the two emitters. We compare this value with the valley splitting of the delocalized excitons of $\mathrm{MoSe}_{2}$ from circular polarization resolved PL studies as a function of magnetic field which is presented earlier in chapter 2, section 2.3.2. Figure 2.8a 
clearly shows the valley splitting of the trion (shaded in black) and neutral exciton peak (shaded in red) for single layer $\mathrm{MoSe}_{2}$. For zero field, there is no significant splitting but it increases with magnetic field. This Zeeman splitting is due to valley dependent magnetic moment [73]. The Zeeman splitting for $\mathrm{MoSe}_{2}$ neutral exciton and trion is presented in Fig. 2.8b and from the linear fit we derive magnitude of g-factor for the charged and neutral excitons to be about 3.9 and 4.2 respectively, which are very similar to the localized exciton peaks. This tells us that the studied localized emitters likely derive some of their properties from the delocalized $\mathrm{MoSe}_{2}$ exciton. In contrast, the single photon emitting centers in $\mathrm{WSe}_{2}$, have large gfactors $(\sim 7-10)$ as compared to their $2 \mathrm{D}$ excitons $[75,76,77,74]$.

\subsection{Gate voltage dependent emission}

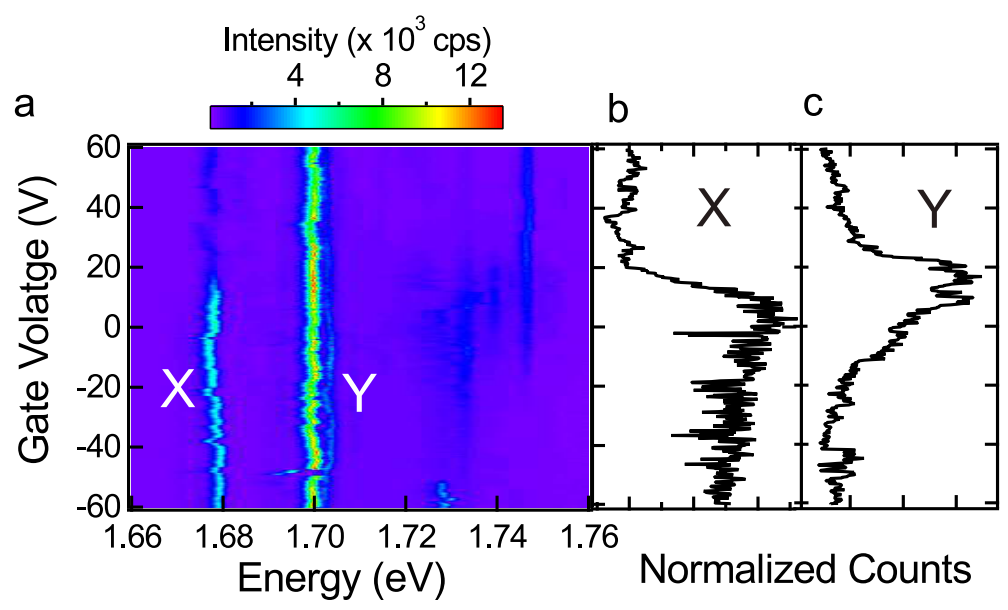

Figure 3.15: Gate voltage dependence: a. $\mathrm{WSe}_{2}$ quantum dot emission spectra as a function of applied gate voltage for two different quantum emitter X and Y. b,c Voltage-dependent photoluminescence for X and Y. A integrated bandwidth of five mev is used to generate each dataset. Figure adapted from [74] with permission.

In this section, we will show that the emission properties of the emitter can be 
modified by applying a gate voltage through the doped silicon backgate. In case of the delocalized excitons (refer to Chapter 2, Section 2.2), neutral excitons have maximum photoluminescence intensity at around zero back gate voltage ie, no electrostatic doping by the charge carriers induced by the gate potential. However, the concentration of charged excitons increases with a finite increase in voltage. As a result, the optical spectral weight is transferred from neutral to charged excitons [67]. Similar to the delocalized excitons in two-dimensions, the localized excitons also demonstrate a gate voltage control. Figure 3.8a presents the gate voltage dependence PL from the emission centers. From the spectrally integrated voltage dependent emission line-cuts in Fig. 3.8b, we observe a reproducible voltage dependent maxima in photoluminescence for feature $\mathrm{X}$ and $\mathrm{Y}$.

\subsection{Strain dependent emission}
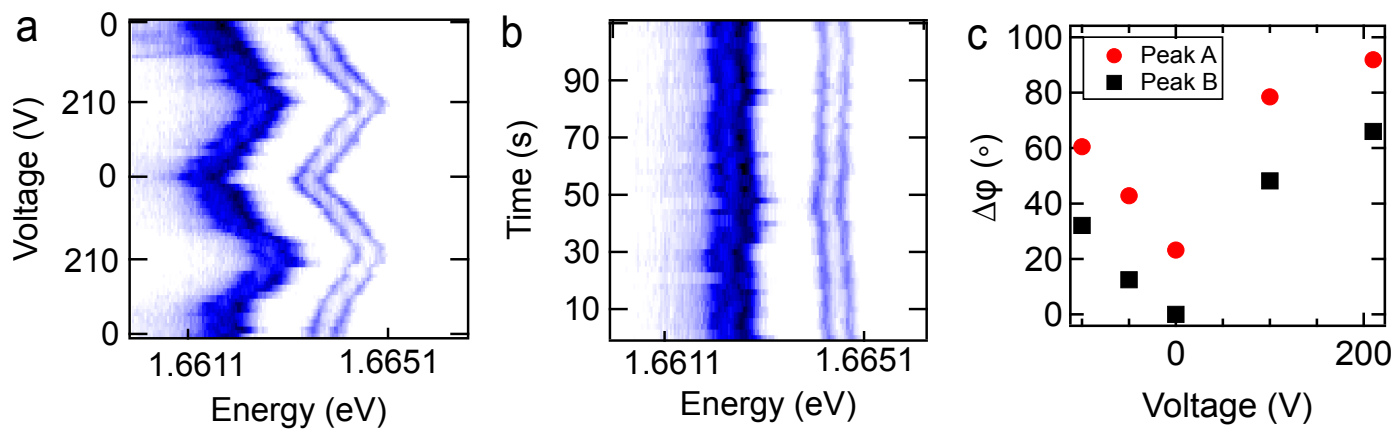

Figure 3.16: Strain dependent emission: a. $\mathrm{WSe}_{2}$ quantum dot emission spectra as a function of applied piezoelectric voltage for two different doublets. b. Time trace of the PL emission at $210 \mathrm{~V}$ c. Change in emission angle as a function of voltage for the doublet emitting at higher energy

One of the most unique properties of 2D materials over conventional semiconductors is their high stretchability of more than $20 \%$ before fracture [107]. Thus, 
strain has been a very interesting knob in the $2 \mathrm{D}$ community as it is a natural way to tune the band gap of a material $[108,109,109]$. In this section, we will study the emission properties of these quantum emitters as a function of strain.

The approach we used to study strain dependence is by simply transferring a monolayer flake on a piezoelectric substrate (PMNT-001 from MTI wafers). Efficient strain transfer to the flake from the substrate is expected due to strong van der Waals interaction of TMDC with the piezoelectric substrate [110]. The piezo substrate is contacted with e-beam evaporated gold electrode on the top and bottom so that a finite voltage can be applied against the electrodes. Remarkably, we observe a shift in the emission energy as a piezoelectric field is introduced across the substrate. In Fig 3.9a, PL color map as a function of the piezoelectric voltage is presented for two doublets. We get a maximum energy shift of $1.3 \mathrm{meV}$ on these quantum dots which can be reversibly applied without significant hysteresis. To demonstrate the stability at the maximum applied voltage $(210 \mathrm{~V}$ or $420 \mathrm{~V} / \mu \mathrm{m})$, we present PL time trace data which in Fig. 3.9b. Further, we also observe a change in orientation of the emission angle of the emitters. We present the data for the two peaks of the doublets around $1.665 \mathrm{eV}$ in Fig. 3.9c. $\Delta \phi$ represents the relative rotation of the linearly polarized emission for the lower and higher energy peaks of the doublets represented by black and red data points respectively. A maximum rotation of $65^{0}$ is observed as the voltage is increased from zero to $210 \mathrm{~V}$.

\subsection{Conclusion}

In summary, we have demonstrated that atomically thin semiconductors can host optically active quantum dot-like emitters. The origin of these emitters is possibly from highly localized strain in the monolayers occurring at visible imperfections 
in the flake like a crack or a fold. These emitters are robust to several power and temperature cycling. They serve as a source of single photons into the bargain that are correlated with the internal electronic structure, much like semiconductor quantum dots and NV centers in diamond. Further, they also demonstrate efficient control via external electric and magnetic field. We envisage a number of future directions enabled by this demonstration. Van der Waals heterostructures will provide a novel platform to demonstrate Stark effect via electric field control which will be discussed in the next section. This will make electrically controlled TMDC quantum dots a viable physical implementation for quantum information processing [111]. Finally, understanding the interplay between the $\mathrm{WSe}_{2}$ quantum dots and the $\mathrm{WSe}_{2}$ excitons will enable new perspectives on valleytronic devices. 


\section{Chapter 4}

\section{Quantum confined Stark effect of single photon emitters in $\mathrm{WSe}_{2}$}

In this section, we study the quantum-confined Stark effect (QCSE) of the localized emitters in monolayer tungsten diselenide [112]. By carefully designing sequences of metallic (graphene), insulating (hexagonal boron nitride) and semiconducting $\left(\mathrm{WSe}_{2}\right)$ two-dimensional materials, we fabricate a field effect device based on van der Waals heterostructure with monolayer $\mathrm{WSe}_{2}$ hosting quantum emitters that are responsive to external static electric field applied to the device. A very efficient spectral tunability up to $21 \mathrm{meV}$ is demonstrated. Further, evaluation of the spectral shift in the photoluminescence signal as a function of the applied voltage enables us to extract the polarizability volume (up to $2000 \AA^{3}$ ) as well as information on the dipole moment of an individual emitter. The Stark shift can be further modulated on application of an external magnetic field, where we observe a flip in the sign of dipole moment possibly due to rearrangement of the position of electron and hole wavefunctions within the emitter. 


\subsection{Introduction}

Controlling the excitonic wave function is a key step to understanding quantum physics and realizing devices with single emitters in these semiconductors. An externally applied electric or magnetic field is normally applied to manipulate the wave functions in a single emitter. In this section, we leverage the quantum confined Stark effect (QCSE) to realize high-resolution voltage controlled optical spectroscopy demonstrating the tunability of the electronic and optical properties of the quantum dot-like localized-excitons embedded in a van der Waals heterostructure. van der Waals heterostructures are an emerging nanoscale optoelectronics device architecture yielding a wide range of device functionality based on stacking two-dimensional materials of various electronic properties as introduced in chapter 1 . These heterostructure offer a platform to realize artificial materials with designable band structures offering an opportunity to study basic phenomenon and develop optoelectronic devices. Some examples of such assemblies are multiple quantum well heterostructures that have been devised to form efficient light emitting diodes [45] and interlayer excitons recombination of electron-hole pair hosted by different TMDC layers [113]. Moreover, heterostructures of 2D materials stacked on elastic and transparent substrates provides basis for flexible and semi-transparent electronics [45]. Such band structure engineering have been previously exploited to create LEDs and lasers based on epitaxially grown III-V semiconductor heterostructure. Previous studies of such quantum well devices showed that the excitons in these devices can be effectively controlled by light and voltage [64]. The question we sought to address was whether we can control the emission properties of the single localized emitters hosted by the TMDC WSe 2 through a voltage applied from a diode like assembly with the van der Waals stacking and demonstrate quantum-confined Stark effect (QCSE). The physical origin 
of QCSE is in the separation of photo-generated charges, creating a dipole that opposes the externally applied electric field. Here, we study the permanent dipole moment arising due to spatial separation of electron and hole wave functions in an exciton trapped within a localized confinement potential, and polarizability, which decides the extent to which an applied electric field can pull the electron's and hole's wavefunctions. Both of the parameters estimate the sensitivity of exciton energy to an applied field. If the polarizability is large in an emitter, its energy can be tuned over a wide range with moderate electric field; therefore, it is lucrative to have large polarizabilities for nanomaterial device applications involving single photon sources and detectors. We utilize QCSE and quantify the wide range of dipole moments and polarizabilities values from several emitters. Further, we also demonstrate that the dipole moment of the emitter can be inverted on application of a static magnetic field perpendicular to the flake. Thus, this provides an alternative pathway to control the charge distribution within the emitter and tune the electron-hole interaction. We anticipate this demonstration of the QCSE of single emitters in a van der Waals heterostructure will benefit their future study and application much like indium arsenide quantum dots [114].

\subsection{Heterostructure assembly and characteriza- tion}

In this section, we will describe the device schematic, its assembly and some of the basic optical characterization of the heterostructure assembly. 


\subsubsection{Device fabrication}

Figure 4.1a displays the schematic ordering of the device. The flakes were mechanically exfoliated and stacked vertically using a PDMS-based all dry transfer method [48] onto pre-patterned $\mathrm{Cr} / \mathrm{Au}$ electrodes on a $\mathrm{Si} / \mathrm{SiO}_{2}$ substrate. The few layer graphene (FLG) flakes on the top and bottom of the heterostructure serve as semi-transparent conductive electrodes. Although not single layer graphene, the flakes are still thin enough to transmit a significant amount of signal from the underlying emitters. Qualitatively similar behavior has been seen on using graphene or graphite as electrode material [115]. WSe ${ }_{2}$ housing the single photon emitters are sandwiches within large band-gap h-BN that form the tunnel barriers. Further, the h-BN layers also separate the $\mathrm{WSe}_{2}$ housing the single emitters from the FLG to minimize quenching of the emitted signal due to energy transfer with graphene [116]. Figure 4.1b displays an optical micrograph of the device with the dashed white box outlining the heterojunction region as illustrated in the schematic of Figure 4.1a. The sample was characterized at low temperature in an attoDRY-1000 cryostat at $4 \mathrm{~K}$ equipped with a confocal microscope. The input light source used for photoluminescence (PL) studies was a continuous wave MOPA laser at $675 \mathrm{~nm}$ wavelength that was focused on the sample using a 0.82 numerical aperture objective. The same objective was used to collect the light emitted from sample. The signal was then directed to a spectrometer with a liquid nitrogen-cooled charge coupled device (CCD) array. For details on the setup refer to section 3.2 of chapter 3 .

\subsubsection{Device characterization}

An exemplary PL spectrum is shown in Fig. 4.1c. Apparent from the image are spectral lines from several emitters in the $\mathrm{WSe}_{2}$. These emitters emit at smaller 
a

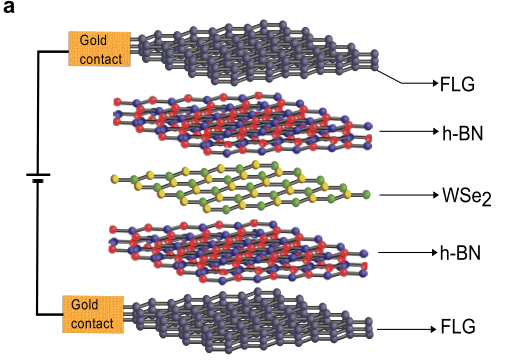

c

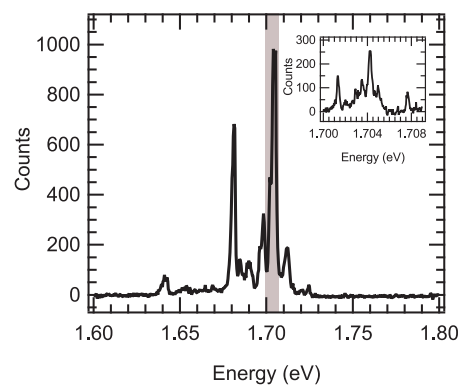

b

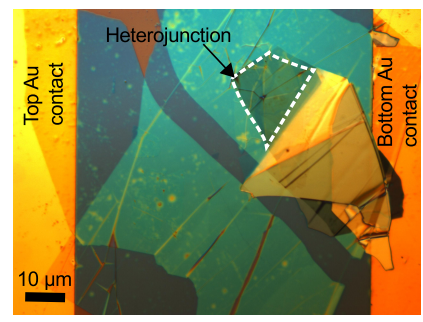

d

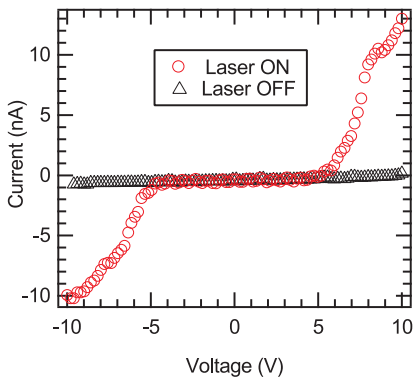

Figure 4.1: Device assembly and characterization: a. Schematic representation showing the order of the 2D materials stacked to fabricate the device. b. Optical micrograph of the device. The white dashed box outlines the heterojunction part of the device. c. Spectrum of quantum-dot-like emitters in $\mathrm{WSe}_{2}$ at $4 \mathrm{~K}$. Inset: Spectrum from highlighted region of $\mathbf{c}$, excitation wavelength $=675 \mathrm{~nm}$, excitation power $=200 \mathrm{nW}$. d. I-V characteristics of the device at $4 \mathrm{~K}$ showing current with and without the laser excitation. Figure adapted from [112] with permission.

energies than the 2D exciton transition of $\mathrm{WSe}_{2}$, which is at $1.75 \mathrm{eV}$ at $4 \mathrm{~K}$ (Refer to section 3.4 for spectra). A zoom in of the spectral region highlighted with the grey box is shown in the inset of Fig. 4.1c demonstrating linewidth of the order of hundreds of $\mu \mathrm{eV}$. The I-V graph of the device showing diode-like character is shown in Fig. 4.1d. 
a

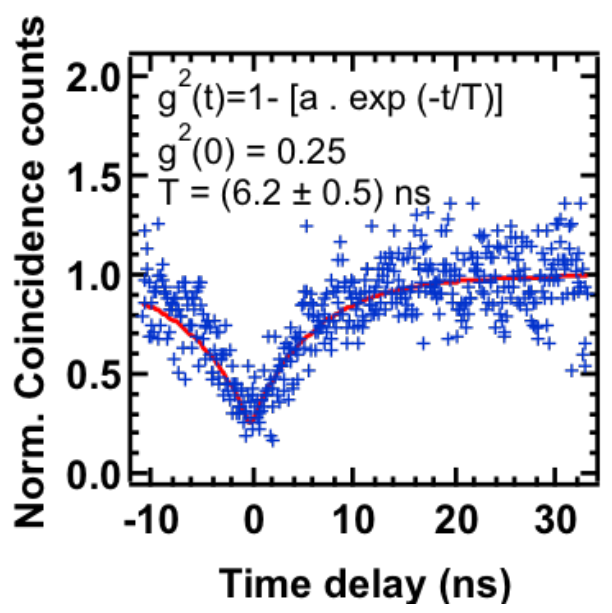

b

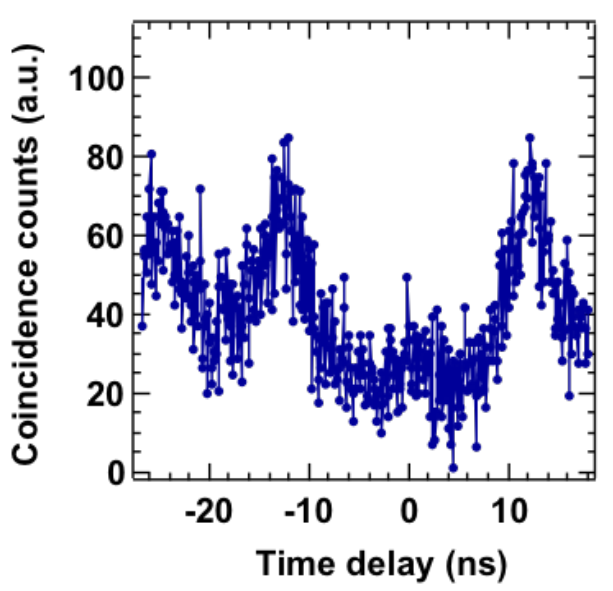

Figure 4.2: Second order intensity autocorrelation measurement using a. continuous wave excitation and b. pulsed excitation. Figure adapted from the supplement of [112] with permission.

\subsubsection{Confirmation of quantum light emission}

For the following spectroscopic studies, the spatial regions of the sample exhibiting the sharp PL emission were first identified as sources of single photon emitters through second order autocorrelation measurements. Intensity autocorrelation measurement was performed using both continuous wave excitation (Fig. 4.2a), and pulsed excitation (Fig. 4.2b) at $620 \mathrm{~nm}$ with repetition rate of $80 \mathrm{MHz}$ (pulse width $\sim 1 \mathrm{ps})$. Both the data displays single photon emission from the presence of a dip at zero-time delay. The measured radiative decay rate extracted from the fit is about $6 \mathrm{~ns}$ for the measured emitter. The coincidence counts are normalized to the points corresponding to value of time delay greater than $25 \mathrm{~ns}$ as obtained in the raw data. 


\subsection{Demonstration of Stark Effect}

In this section, we will demonstrate and confirm the Stark effect on the single emitters in $\mathrm{WSe}_{2}$. We will also extract the values of permanent dipole moment and polarizability by fitting the data. Finally, based on statistics, we will study the trend of some of these fitting parameters with respect to the emission energy of the emitters.

\subsubsection{Photoluminescence vs vertical electric field}

Following the confirmation of quantized emission, PL was recorded at these locations as a function of applied voltage. Figure 4.3a displays an exemplary spectral map as the applied voltage is swept in predetermined steps. Clear in the PL spectra is a rich variety of spectral responses exhibited by the emitting centers most likely due to different structure and size of the emitters. Such spectral shifts are a manifestation of the QCSE in the quantum dot-like emitters present in 2D materials when assembled into such heterostructure device. In our earlier device in section 2.2 where the TMDC was directly contacted with the electrode and the silicon back contact was used to gate the device, slight blue shift of the charged delocalized excitons was observed likely due to band filling effects. However, PL energy shift of the localized excitons was not observed in such back-gated device assembly (Refer to section 3.8 or [74]).

When sweeping the voltage on the device from negative to positive direction, we observe that along with a spectral shift there is a modulation of the linewidth and PL intensity from the emission lines. The data in Fig. 4.3b illustrates this effect for the transitions identified by the red box in the adjacent spectral maps. As the voltage is swept from negative to positive, the line width decreases with a cor- 
a
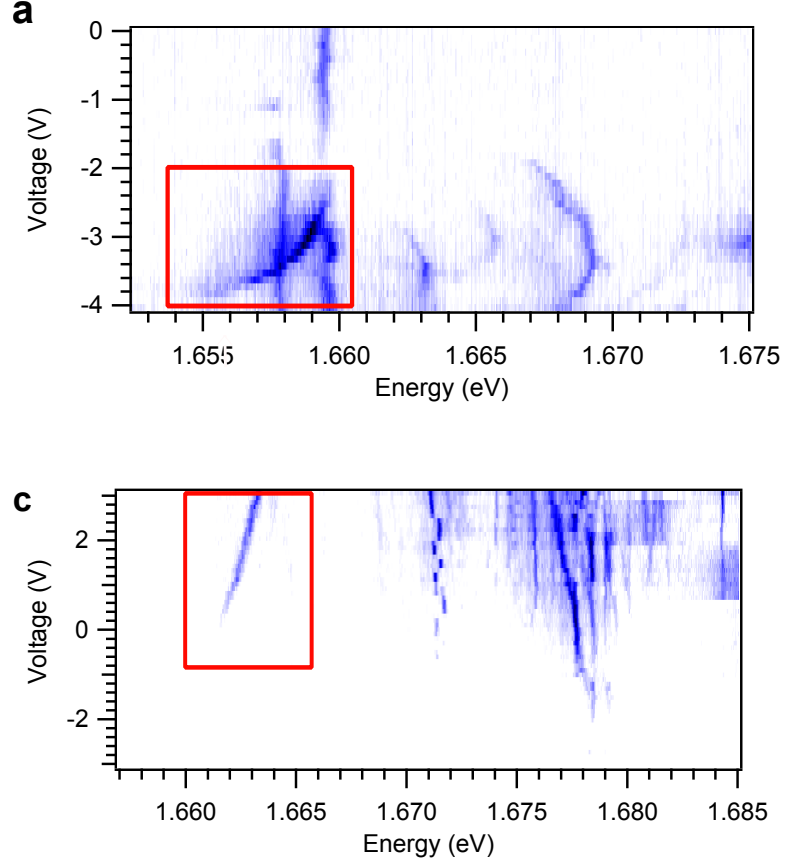

b

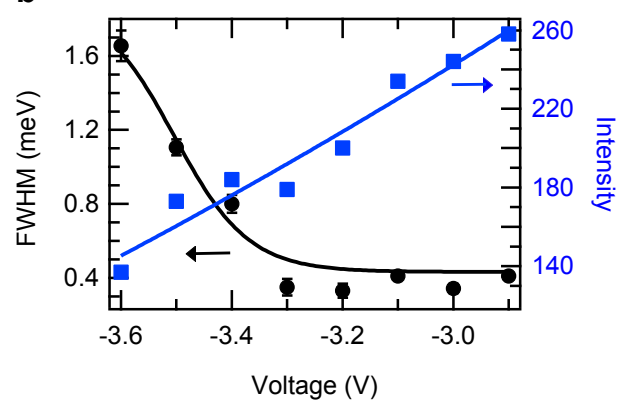

d

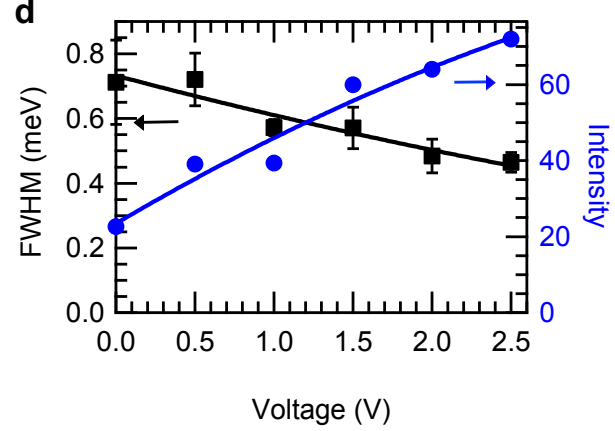

Figure 4.3: PL vs voltage: a, c. Spectral map showing the Stark shift of various emitters under applied field from two different locations of the sample. $\mathbf{b}, \mathbf{d}$. The spectral linewidth as measured by full width at half maximum (FWHM) and intensity of the emissions as a function of the applied voltage. The notated emitters in $\mathbf{a}$ and $\mathbf{c}$ correspond to the data presented in $\mathbf{b}$ and $\mathbf{d}$, respectively. In Fig. a, we study this effect for the emission line varying from $1.657 \mathrm{eV}$ (@ -3.6 V) to $1.669 \mathrm{eV}$ (@ -2.8 V). Bold lines are guide to the eye. Figure adapted from [112] with permission.

responding increase in the PL intensity. This suggests a decrease in non-radiative processes such as tunneling. Such spectral shift and linewidth modulation due to the external bias are distinctive features of the QCSE. These observations reflect the buildup of an internal electric field or dipole that opposes the externally 
applied field also observed earlier with other confined excitonic systems $[117,118]$.

\subsubsection{Spectral wandering vs Stark shift}

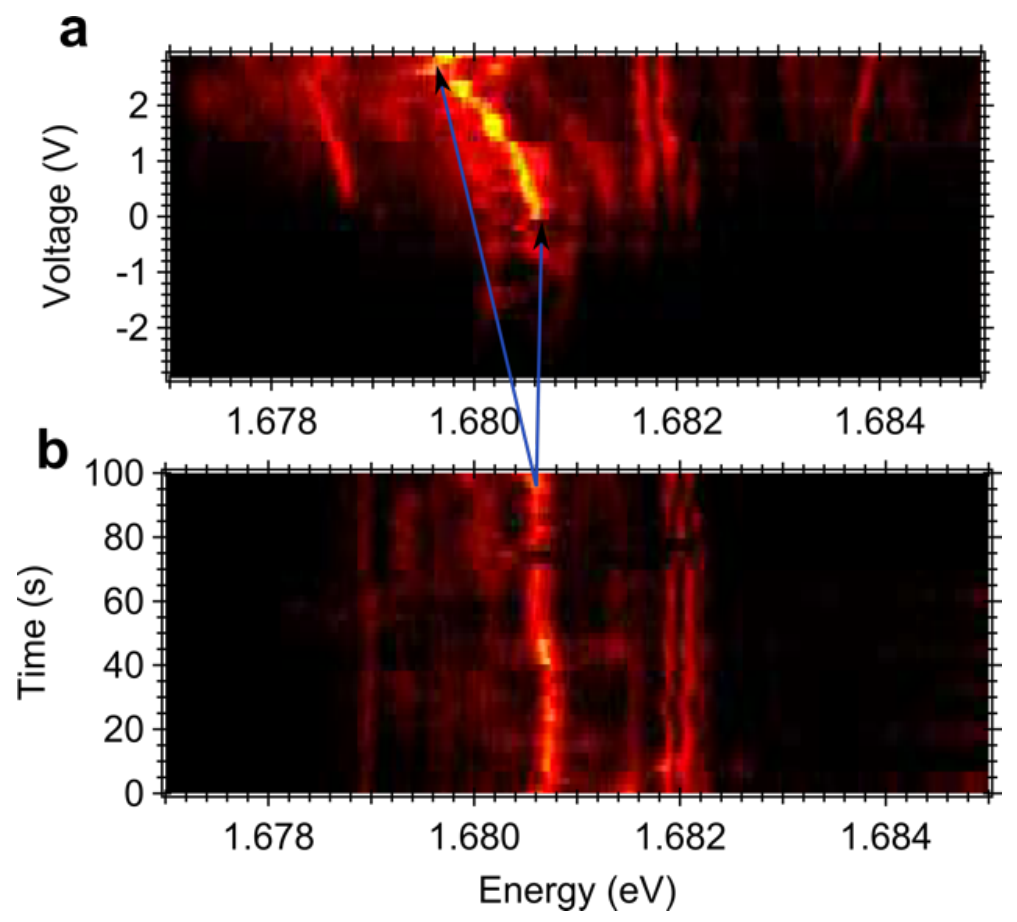

Figure 4.4: Spectral diffusion vs Stark shift: a. Stark shift as a function of applied voltage. b. Intensity and spectral fluctuations of the same emission lines as a function of time at zero electric field. Figure adapted from the supplement of $[112]$ with permission.

To verify that the observed shifts are due to the changing applied voltage bias and not due to random spectral shifts, it was confirmed the PL spectral wandering was at most $150 \mu \mathrm{eV}$, which is much less than the voltage-controlled shift. For the case presented in Fig. 4.4a the maximum shift in emission energy for the exemplary case of the emitter around $1.68 \mathrm{eV}$ is about $1 \mathrm{meV}$ due to the controllably applied external electric field. More than $20 \mathrm{meV}$ electric field 
induced spectral shift have been observed for certain cases (Fig 4.5a). This is almost an order of magnitude larger than the spectral wandering within a 100 second time period (Fig 4.4b) of the same region where the maximum change in energy is within a window of $150 \mu \mathrm{eV}$.

\subsubsection{Fit to Stark shift equation}

The spectroscopic utility of the QCSE is that it reveals the exciton's built in dipole moment and polarizability and these can be found by fitting the PL emission energy (E) to the following equation:

$$
E=E_{0}-\mu F-\frac{1}{2} \alpha F^{2}
$$

where $E_{0}$ is the zero-field transition energy, $F$ is the local electric field acting at the emitter, and $\mu$ and $\alpha$ are the dipole moment and polarizability, respectively, between the ground and excited states. Similar to an approach followed previously [119], $F$ is calculated from the applied voltage $(\mathrm{V})$ by the Lorentz local field approximation, $F=V(\epsilon+2) / 3 / t$, where $\epsilon$ is the dielectric constant and $t$ is the thickness for the surrounding h-BN environment. Our devices are approximately $30 \mathrm{~nm}$ thick, and the dielectric constant of h-BN is taken to be 3 [120].

Figure $4.5 \mathrm{a}$ presents the optical transition energy as a function of applied voltage taken from an emitter exhibiting a linear Stark shift. We have observed a giant energy shift of $21 \mathrm{meV}$ from this emitter which is comparable to Stark shifts obtained from InAs/GaAs quantum dots in a heterostructure device [121]. About $15 \%$ of the studied emitters show a Stark shift within this order of magnitude. The dipole moment $(\mu)$ of this emitter is calculated to be $-2.4 \mathrm{D}$, where $1 \mathrm{D}=3.33 \times$ $10^{-30} \mathrm{C} \cdot \mathrm{m}$. Predominantly linear Stark effects have been observed from about $31 \%$ of the emitters. This kind of behavior is expected from emitters which lack a center 

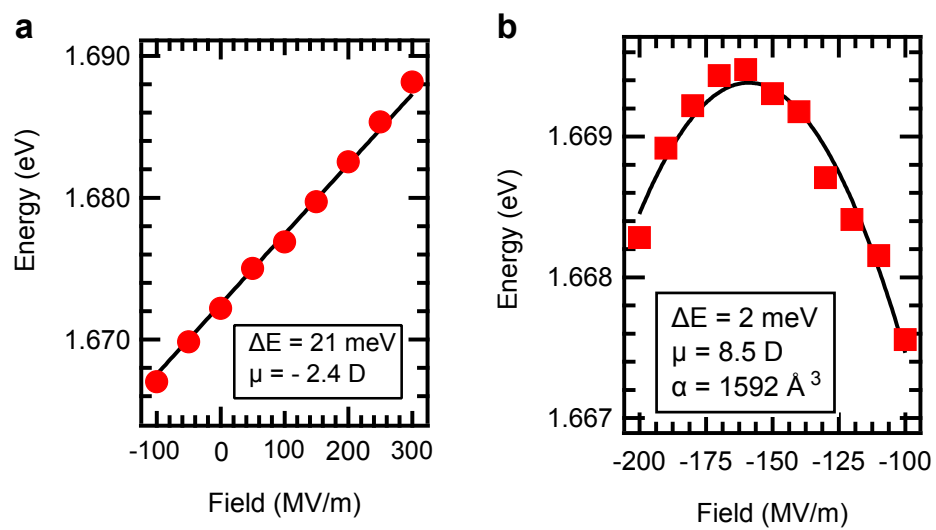

Figure 4.5: Fit to Stark shift for two different emitters displaying a. linear Stark effect; b. linear and quadratic Stark shift. Figure adapted from [112] with permission.

of symmetry. Figure $4.5 \mathrm{~b}$ shows an emitter with a strong linear and quadratic component; the polarizability volume is $\alpha=1592 \AA^{3}$. The polarizability volume is the polarizability divided by a factor of $4 \pi \epsilon_{0}$. The changes in polarizability are expected to be of the order of the emitter center volume [119]. Among the emitters exhibiting Stark shift, 50\% demonstrated a combination of linear and quadratic shifts.

Few of the emitters showed a 'V' shaped Stark effect. An emitter showing symmetric linear Stark shift centered around a finite field $(-119 \mathrm{MV} / \mathrm{m})$ is shown in Fig. 4.6a. It is also observed that about $17 \%$ of the emitters have different dipole moment and polarizability for positive and negative field directions. Example of two such emitters are shown in Fig 4.6c and d, where the stark effect is either linear (Fig 4.6c) or quadratic (Fig 4.6d) on the positive field side and there is zero shift in energy on the negative applied voltage. The dipole moment is almost zero for the negative direction but is finite for the positive. Such effects have been observed previously in chromium related centers in diamond [122].

An important observation made possible by the QCSE is that almost all of 
a

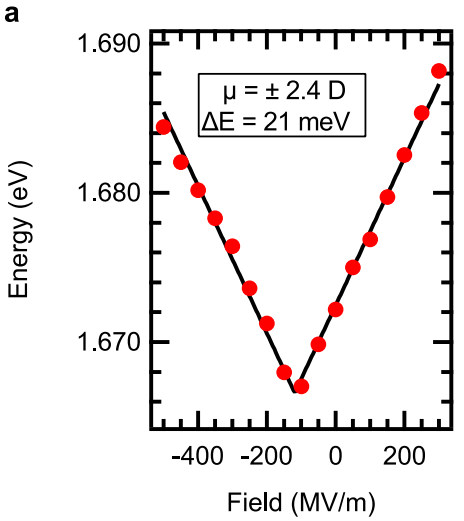

C

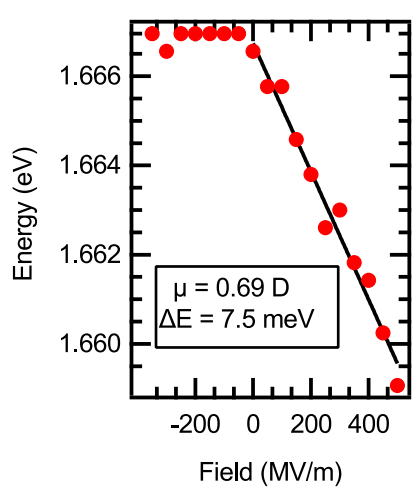

b
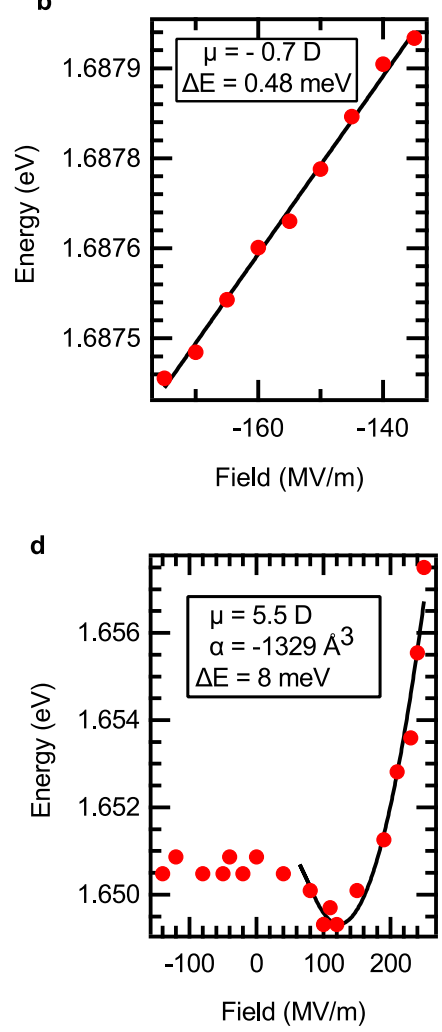

Figure 4.6: Variety of Stark shift trends from different QD like emitters: a. Vshaped Stark Effect. b. Linear Stark shift c., d. Asymmetric Stark effect from emitters around zero field. Figure adapted from the supplement of [112] with permission.

the studied emitters have a built-in dipole moment. This is evidenced from the contribution from the linear component of equation 4.1. The nonzero shift at zero electric field demonstrates a finite built-in dipole moment that has magnitude that varies from $0.15 \mathrm{D}$ to $8.5 \mathrm{D}$. For comparison, a nitrogen vacancy (NV) defect center has dipole moment distribution between $-1.5 \mathrm{D}$ to $1.5 \mathrm{D}$ [119]. From the dipole moment values, we determine that the electron-hole separation within the emitter ranges from $3 \mathrm{pm}$ to $177 \mathrm{pm}$ for all the studied emitters. 


\subsubsection{Statistics}

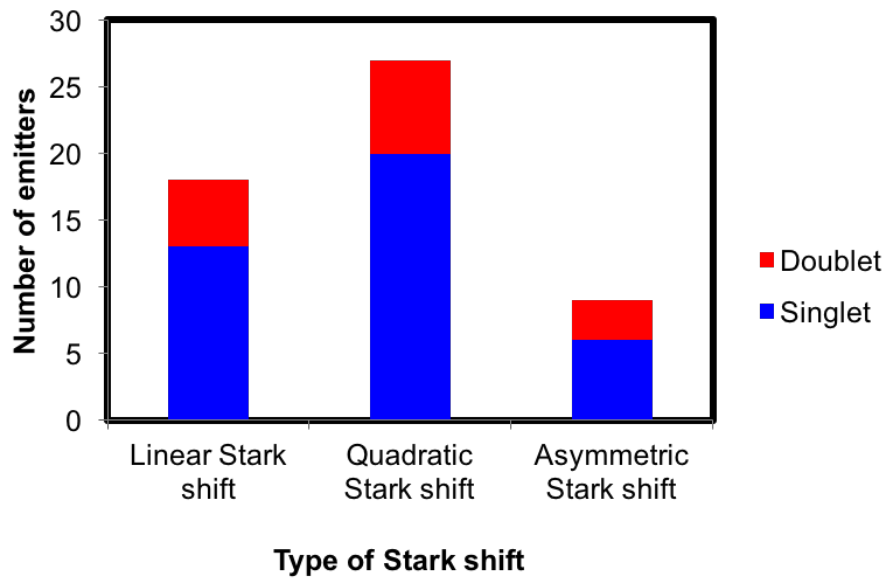

Figure 4.7: Statistics of different varieties of Stark shift. Figure adapted from the supplement of [112] with permission.

A statistical analysis of the different variety of stark effect exhibited by the various studied emitters is presented in Fig. 4.7. Emitters exhibiting doublet and single emission lines are represented by red and blue bars respectively.

Next, we calculated the distribution of total shift in energy $\left(\triangle \mathrm{E}=\mathrm{E}_{\max }-\mathrm{E}_{\min }\right)$ per unit voltage change $\left(\triangle \mathrm{V}=\mathrm{V}\left(\mathrm{E}_{\max }\right)-\mathrm{V}\left(\mathrm{E}_{\min }\right)\right)$ for several emitters and plotted them in Fig. 4.8a. In Fig. 4.8a and b, the magnitude of the shift per unit voltage and, hence, the dipole moment obtained from fitting increases with decreasing energy (increasing wavelength). Also, the polarizability volume (Fig. 4.8c) increases with decreasing energy. This reflects the fact that similar to quantum dots, the emitters at lower energy (longer wavelength) would have a larger volume than a emitter at higher energy (shorter wavelength). As a consequence, the confinement is less pronounced and applied electric fields are more effective at displacing the electron and hole wavefunctions resulting in larger QCSE.

As seen from the statistical analysis in Fig 4.9, majority of the emitters in the 

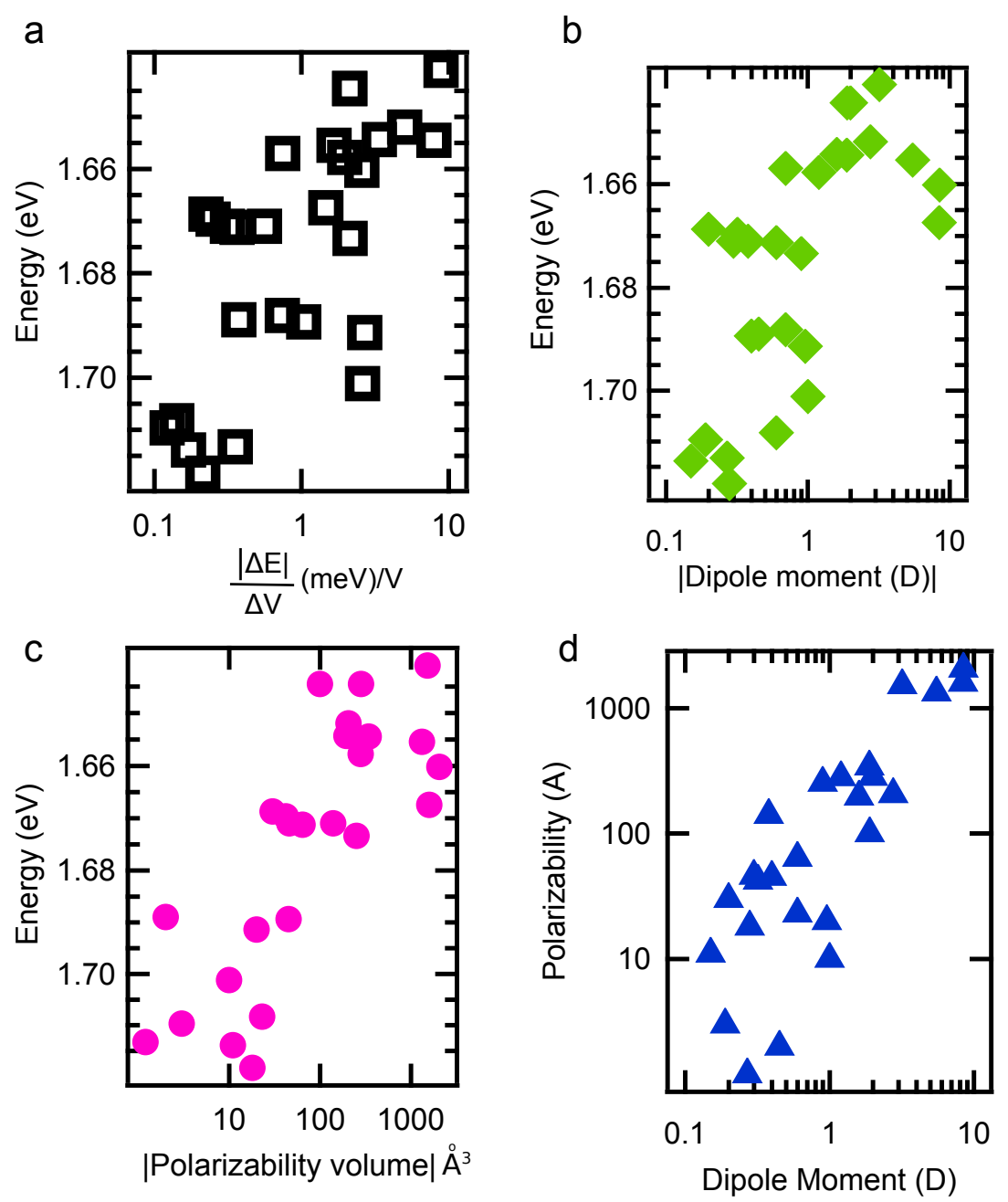

Figure 4.8: Trend of the fit parameters: a. Magnitude of Stark shift $(\triangle E)$ per unit voltage $(\triangle V)$ plotted as a function of the emission energy for the studied emitters. b. Dipole moment $(|\mu|)$, and c. polarizability volume $(|\alpha|)$ vs PL energy of the emitters d. Polarizability volume $(|\alpha|)$ vs dipole moment $(|\mu|)$ for the studied emitters. Figure adapted from [112] with permission.

higher energy range $(>1.66 \mathrm{eV})$, have lower QCSE (grey bar). Together with the size, the symmetry of the emitter could also play a role here. In case of a large asymmetric emitter, charge separation is enhanced, leading to reduced Coulomb attraction between the separated carriers or electron-hole wavefunctions of the 


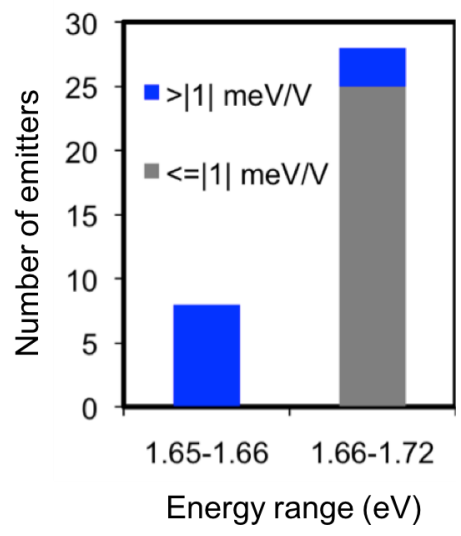

Figure 4.9: Statistical analysis: Energy shift per unit voltage for emitters in different spectral range. Figure adapted from the supplement of [112] with permission. exciton. This also leads to higher response to an electric field [118]. Whereas, for emitters with smaller size and symmetric shape, charge separation will be limited. Next, in Fig. 4.8d, we also see that the polarizability volume $(\alpha)$ and dipole moment $(\mu)$ are correlated and present a nearly linear relationship. This suggests that the built-in field among the several emitters is roughly constant as by classical definition of dipole moment, $\mu=\alpha$.F, where $\mathrm{F}$ is the built-in field. Such correlations have been also observed in InAs/GaAs quantum dots and have been attributed to a roughly constant lateral extent of the excitons irrespective of the quantum dots. [123] However, details of the lateral excitonic confinement is not well understood for the case of these localized single emitters and requires further study.

\subsection{Influence of the magnetic field}

Finally, we studied the effect of a magnetic field perpendicular to the plane of the heterostructure (Faraday geometry). First, the Zeeman effect was studied 
a

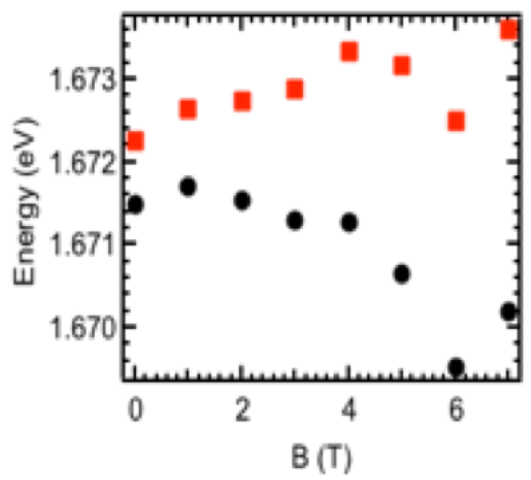

b

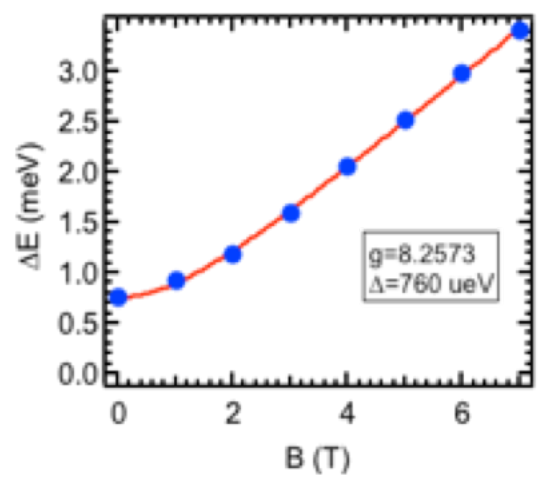

Figure 4.10: Magneto-optical studies: a. Spectral position of the two peaks of the doublet from the defect studied in figure 4 of the main text. b. Separation in energy of the doublet as a function of magnetic field fit to a hyperbolic function. Zero field splitting $(\Delta)$ from the fit is $760 \mu \mathrm{eV}$ and $\mathrm{g}$ factor is $\sim 8$. Figure adapted from the supplement of [112] with permission.

at zero electric field (Fig 4.10a) which showed an increase in the splitting of the doublet features. This is consistent with previous results from magneto-optical spectroscopy (Refer to section 3.7.2) The g-factors for the emitter is calculated to be $\sim 8$ (see Fig 4.10b). For each magnetic field, we study the Stark shift of the doublet as a function of the applied electric field. Both the peaks of the doublet exhibit similar Stark effect. In Fig. 4.11a, the Stark shift for different magnetic fields is plotted for the lower energy peak of the doublet for clarity. Figure 4.11b shows the peak energies extracted at zero electric field. From the linear and quadratic dependence of the Stark shift, the dipole moment (Fig. 4.11c) and polarizability (Fig. 4.11d) are extracted from the fits. At zero magnetic field, the emitter has a positive dipole moment (electron-hole separation) value of $0.04 \mathrm{D}$ $(0.8 \mathrm{pm})$ which then decreases and becomes negative with the maximum value of 
a
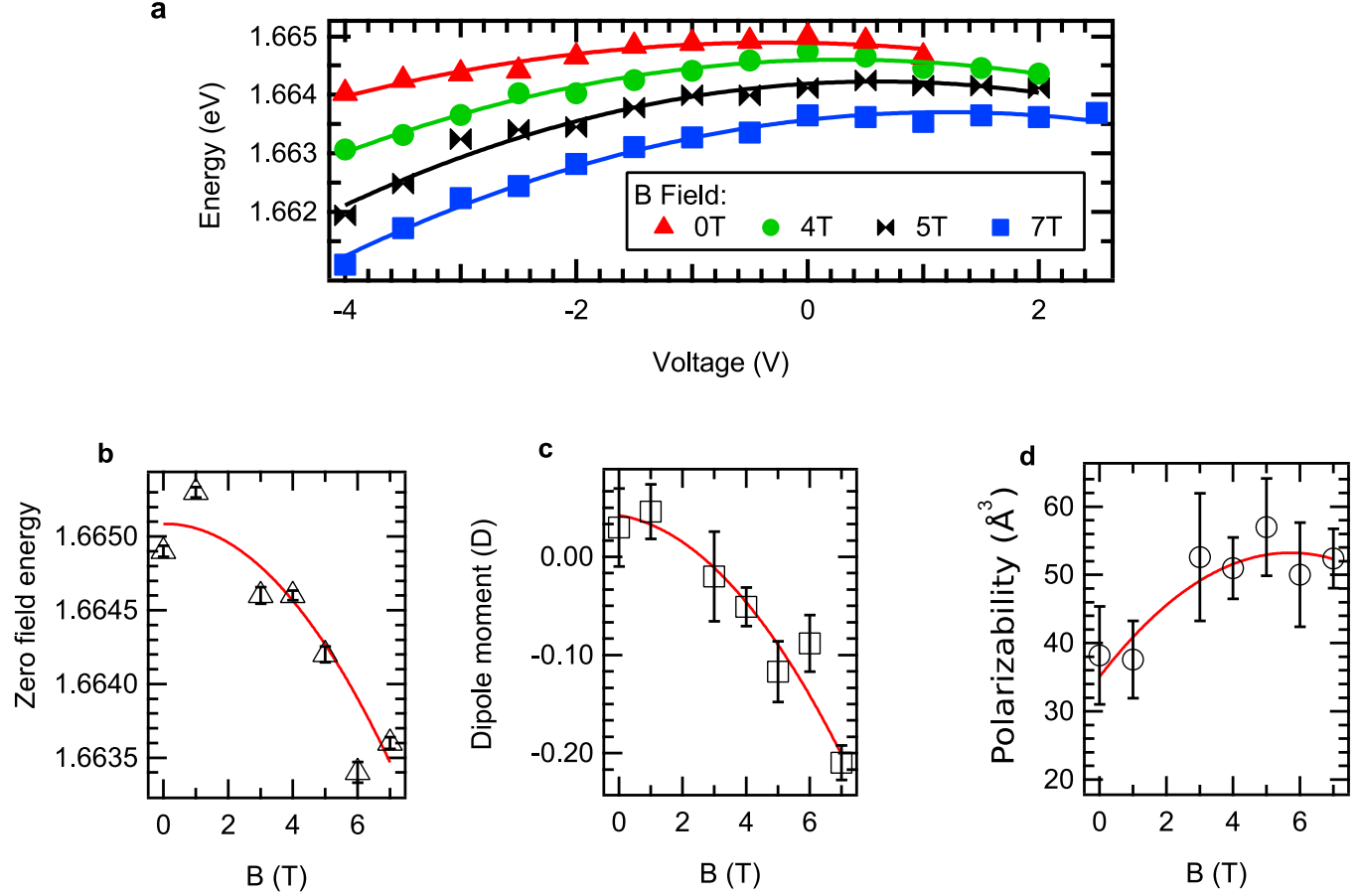

Figure 4.11: Stark effect vs B field: a. Effect of magnetic field on Stark shift. Solid lines are fit to data using equation (4.1). b. Zeeman Effect on the zero electric field energy $E_{0}($ in $\mathrm{eV})$ on the lower energy peak of the doublet, c. Dipole moment flipping sign from positive to negative as magnetic field is increased, $\mathbf{d}$. Effect on polarizability volume (in $\AA^{3}$ ) of the emission center with magnetic field. Error bars indicate fitting error. Solid red lines are guide to the eye in $\mathbf{b}-\mathbf{d}$. Figure adapted from [112] with permission.

$-0.2 \mathrm{D}(4 \mathrm{pm})$ at magnetic field of $7 \mathrm{~T}$. A flip in the sign of the dipole moment was observed along with a change of an order of its magnitude as the magnetic field increased from zero. However, the polarizability of the emitter retained the same order of magnitude with magnetic field. This effect has been attributed to the altering of the confinement potential of the electrons and holes in the quantum dot [124]. Magnetic field can induce carrier redistribution in the quantum emitters which can lead to changes in the permanent dipole moment of the emitter and 
hence the orientation of the dipole with respect to the electric field. Thus, this has shown another pathway of controlling the charge distribution in the single emitter.

\subsection{Summary}

In summary, we have explored the different trends of quantum confined Stark effect on single emitters in atomically thin $\mathrm{WSe}_{2}$. Based on the varied linear and quadratic nature it is clear that atomically thin $\mathrm{WSe}_{2}$ supports different types of optically active quantum emitters. The van der Waals heterostructure field effect type devices has allowed efficient control of the electronic resonances and provided insight into the QDs optical properties. Application of an external magnetic field can alter the QCSE by manipulating the alignment of electron-hole wavefunctions. This has been demonstrated through the inversion of dipole moment as a function of magnetic field. This device concept can be used in future to resonantly tune the transition of an emitting center for strongly interacting with another local emitter $[125,126]$ or a cavity mode [127]. Devices capable of producing an in-plane electric field may help to reveal more information about properties like emitter orientation. Future work utilizing polarization-resolved methods along with voltage controlled spectroscopy will allow for the understanding of the interaction between the $\mathrm{WSe}_{2}$ emitters and $\mathrm{WSe}_{2}$ excitons, paving the way for new devices based on confined valleytronics. Furthermore, precise manipulation of the fine structure splitting from the doublets within such heterostructure devices can also be very useful for solid state quantum information processing, such as, polarization entangled photon pair generation [128]. 


\section{Chapter 5}

\section{Manipulation of the fine}

\section{structure splitting of the}

\section{quantum dot exciton}

Semiconducting quantum dots are considered to be one of the most promising sources of non-classical light as they can generate on-demand single photons. However, their performance as an entangled photon source is still limited which is one of the requirements on the wishlist for applications in emerging quantum technologies [129]. One of the ways to generate entangled photon pairs in QDs is to utilize the biexciton to exciton radiative cascade [128]. However, in semiconductor QDs, the presence of a fine structure splitting (FSS) in their emission states limits the indistinguishability of the emitted photon pairs. The decrease in the symmetry for the quantum dots leads to the mixing of their excitonic spin states due to the anisotropic electron-hole exchange interaction. Further, this leads to an energetic splitting in the emission of the neutral exciton. This is called the fine structure splitting. The magnitude of the FSS depends on the shape, anisotropic strain, composition and the crystal inversion symmetry [128]. 
For generation of entangled photon pairs, the FSS needs to be elimintated. External perturbations like strain, electric field and magnetic field or sometimes a combination of different knobs are used to erase the FSS [130, 131]. FSS is also observed for the case of the quantum emitters in $\mathrm{WSe}_{2}$. In this chapter, we will demonstrate control of the fine structure splitting in some of the emitters by applying a vertical electric field.

\subsection{Modulation of the fine structure splitting}
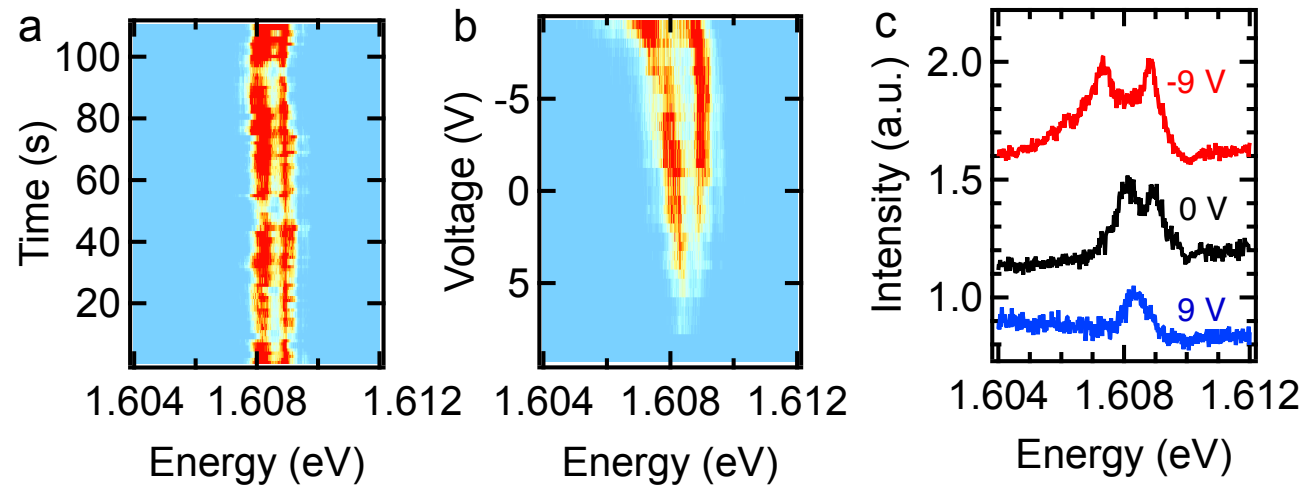

Figure 5.1: Modulation of FSS: a. Time trace of PL from the studied doublet peak shwoing correlated spectral wandering $\mathbf{b}$. PL as a function of voltage showing modulation of the splitting c. Spectral linecut at three different voltages.

The device used in this experiment is a van der Waal's heterostructure with the optically active QDs in $\mathrm{WSe}_{2}$ embedded within the stack. Refer to section 4.2.1 for details on the device geometry. This device is capable of applying an electric field which is sensed by the emitters in $\mathrm{WSe}_{2}$. Fig 5.1a presents the PL spectral map as a function of time. From the map, we see two split peaks which exhibit a correlated spectral wandering. This suggests that the two peaks originate from the same exciton feature. The splitting is between the two peaks gives the 
value of the fine structure splitting to be around $800 \mu \mathrm{eV}$ at zero voltage. This is consistent with the splitting observed in other reports [75, 74, 76] (Refer to section 3.11 about the origin of this splitting in TMDC QDs).
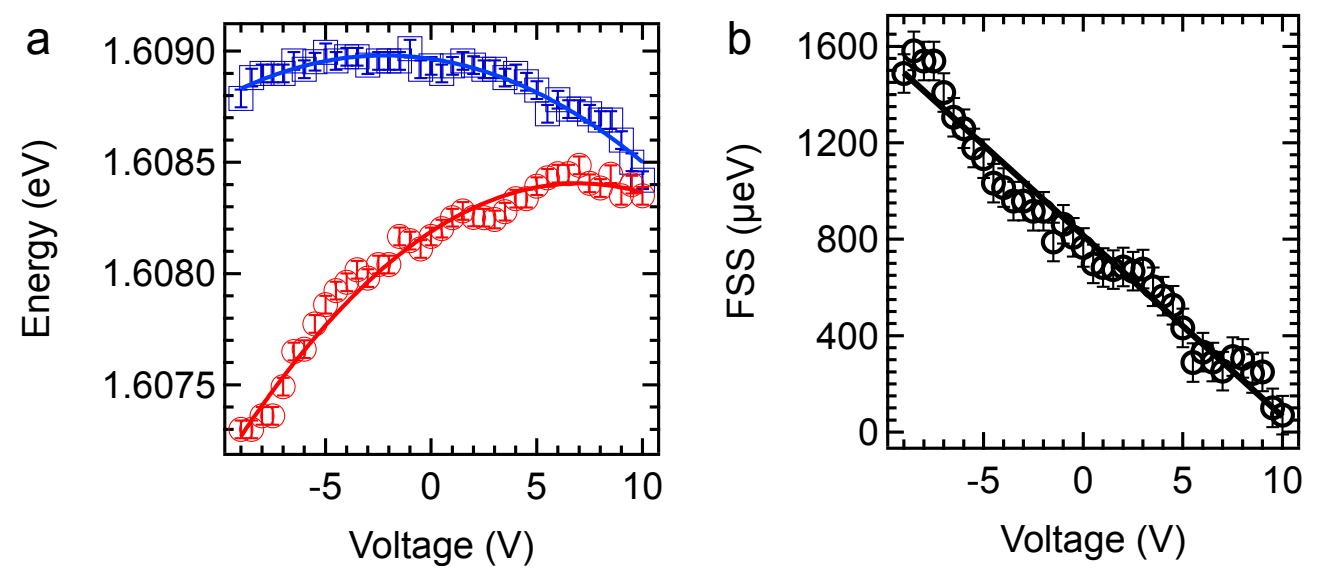

Figure 5.2: Analysis of the doublets: a. Extracted peak energies of the doublet as a function of voltage. Solid lines are fit to Stark shift equation $4.1 \mathrm{~b}$. FSS as a function of applied voltage

Next, we study the correlation between the FSS and the applied electric voltage. Figure $5.1 \mathrm{~b}$ presents the voltage dependent PL map of the doublet showing a modulation of the emission energy of the doublets. This is also clear in the spectral linecuts from three different voltage points plotted Fig. 5.1c. In Fig. 5.2a, we have plotted the extracted peak positions obtained from the color plot in Fig. 5.1b. It is clear from Fig. 5.2a that the Stark effect introduces a shift in the emission energies of the doublets. The Stark effect on various other emitters in $\mathrm{WSe}_{2}$ is also observed in chapter 4 . The two lines of the doublets are fit to the Stark effect equation 4.1 described in chapter 4 . It is well known that the Coulomb exchange interaction can be modified by the Stark effect as the electric field can modify the overlap of the carriers wavefunctions [132]. In this case, we see that 
both the eigenstate of the neutral exciton shift at different rates as a function of the applied electric field. This is possibly due to the orientation of the dipole with respect to the electric field which affects the two split states unequally along the electric field direction. This leads to a linear change in the splitting (Fig. 5.2b) observed up to the resolution limit of our setup which is $(0.02 \mathrm{~nm}$ or $40 \mu \mathrm{eV})$. The orientation of the emitters polarization with respect to the electric field strongly affects the fine structure splitting. Due to this, two different controls are used in earlier experiments (combination of strain and electric field) [133], where one perturbation is used to align the orientation of the exciton along the axis of the other control knob for complete reduction of the fine structure splitting of the doublet emission state. For the case of epitaxially grown QDs, strain has been often used to align the QD exciton along a preferred direction of the electric field where the effect of the electric field will be maximized. Both the strain and electric field act as effective deformation to restore the symmetry of the quantum dots. The emitters embedded in monolayer $\mathrm{WSe}_{2}$ can be under the influence of random strain fields due to imperfections in the exfoliated crystal which may or may not align the emitter along the electric field. This is why we see this effect only in $10-15 \%$ of the emitters studied in the different heterostructure devices which also vary from emitter to emitter.

\subsection{Circular polarization vs FSS}

As the finite spectral resolution of the experimental setup does not let us prove $\mathrm{FSS}=0$, we measure the circular polarization of the exciton peaks as complete reduction in the degeneracy should imply maximum polarization of the exciton 

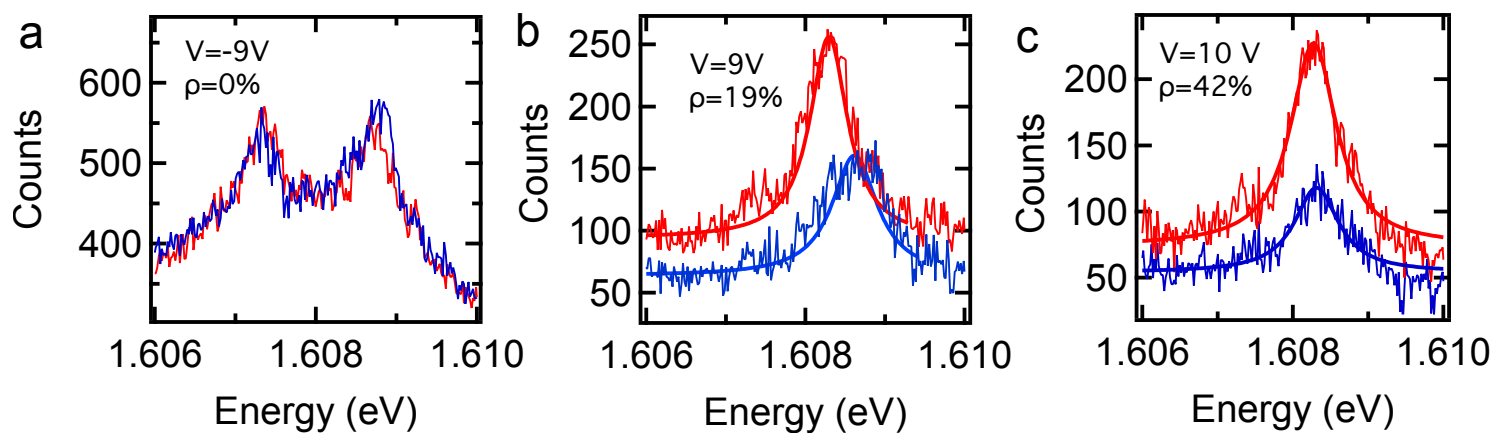

Figure 5.3: Circular Polarization: a,b,c Circular polarization resolved PL spectra at different voltages: $-9 \mathrm{~V}, 9 \mathrm{~V}$ and $10 \mathrm{~V}$ respectively. Red (Blue) curve is measured with $\sigma^{+}\left(\sigma^{-}\right)$basis.

state. The degree of circular polarization is defined as:

$$
\rho=\frac{I_{\sigma^{+}}-I_{\sigma^{-}}}{I_{\sigma^{+}}+I_{\sigma^{-}}} * 100 \%
$$

where $I_{\sigma^{+}}$and $I_{\sigma^{-}}$are intensities for $\sigma^{+}$and $\sigma^{-}$detection. Circularly polarized resolved PL spectra at different input voltages are shown in Fig. 5.3. The degree of circular polarization is expected to increase as the fine structure approaches zero [134]. In this case the neutral exciton is no longer a mixture of the spin-valley states as the FSS approaches zero. In Fig 5.3a, the PL lines are unpolarized in the circular basis. Increasing the voltage in positive direction leads to an enhancement in the degree of circular polarization only up to $42 \%$. Although this indicates that a FSS is still present, it could not be detected any further due to the resolution limit of the setup. The spectral resolution can be improved by using a Fabry-Perot interferometer which is a direction of future study. On increasing the voltage beyond $10 \mathrm{~V}$, the device switches to the tunneling regime for this QD where, the confinement energies have been reduced to the extent that the electron-hole pairs tunnel out from the emitter. Therefore, we are limited by the confinement energies which limits us from applying higher field which could 
potentially restore the symmetry of the emitter to provide unity degree of circular polarization.

\subsection{Summary}

In summary, we have demonstrated the manipulation of the FSS by applying an external voltage. This has been further confirmed by the enhancement in the degree of circular polarization due to reduced mixing from electron-hole Coulomb exchange interactions. Such electric field devices are well suited to be integrated to low volume cavities enabling higher efficiencies and cavity QED effects with large Purcell enhancements [135]. In future, a field effect device with deterministically created quantum dots fabricated on a piezoelectric substrate or flexible substrate can be employed to apply a combination of strain and electric field to observe more versatile and effective modulation of the FSS. With sufficient suppression of the FSS, the cascaded emission from biexciton [98] can be utilized as a source of entangled photons in scalable solid-state based quantum information technology. 


\section{Chapter 6}

\section{D localized exciton}

\section{complexes in a charge tunable}

\section{van der Waals heterostructure}

In this chapter, we investigate fully localized excitons and trions from the quantum emitters in tungsten diselenide embedded in a charge tunable van der Waals heterostructure [136]. We use direct electrostatic doping through the vertical heterostructure device assembly to generate quantum confined trions. Distinct spectral jumps as a function of applied voltage bias, and excitation power dependent charging, demonstrate the observation of different excitonic complexes. We also observe a reduction of the intervalley electron-hole exchange interaction in the confined trion due to the addition of an extra electron which is manifested by a decrease in the fine structure splitting (FSS) for the case of the charged exciton. We further confirm this decrease of exchange interaction for the case of the charged states by a comparative study of the circular polarization resolved photoluminescence from individual excitonic states. Recovery of the valley polarization of these localized trions demonstrates the possibility of a spin-valley-photon interface through the optically active charged excitonic complexes in these quantum 
dots.

\subsection{Introduction}

The operation of electronic devices is made possible by the electron's charge. Recent advances in nanoscience have created the opportunity to leverage the electron's spin in future technologies [137, 138]. It is envisioned that spin can serve as a store of information $[139,140]$ or provide a vehicle for high resolution sensing $[141,142]$. Of particular interest is electron spin in quantum confined nanomaterials that support optical transitions. In the previous, photons link spin ground states and trion excited states [143]. In this work, we present the observation of $3 \mathrm{D}$ confined trions in a van der Waals heterostructure. A single layer of tungsten diselenide hosts quantum dot (QD) like emitters attributed to defects $[74,75,76,77,78]$ or localized strain pockets in the flakes $[86,85,91,84]$ as discussed in chapter 3. Here, we demonstrate voltage controlled QD charging enhancing the valley polarization of the localized trion complex. The observation of $3 \mathrm{D}$ confined trion in a van der Waals heterostructure will present new opportunities for quantum photonics and provide an interesting environment to study confined spin-valley physics.

The emergence of TMDCs as materials for nano-optoelectronics has focused attention on the existence and stability of their neutral and charged excitons [52]. Interesting in TMDCs is the symmetry mediated strong photon polarization selection rule that interfaces the two inequivalent $\mathrm{K}$ points of the Brillouin zone with distinct circular polarization orientations. As a result, it has been proselytized that the valley index may provide a novel information bearing degree of freedom [65] and its optical manipulation has been explored. Important for single spin 
studies in TMDCs is that photon energy and polarization can selectively excite specific spin ground state/trion excited state combinations [52].

The recent discovery of localized QD in single layer TMDCs has catalyzed investigations into 3D localized exciton resonances in these materials [81, 144, 98]. As a result there has been intense interest as to whether it may be possible to controllably charge the TMDC-QDs. Such controlled charging would engender the QDs with stable spin ground states that could be utilized as qubits or nanoscale sensors. Further, under appropriate conditions, the QD can inherit the strong polarization selection rules of the host material providing a robust spin-valley-photon interface. Previously, a complete recovery of valley polarization was demonstrated in neutral excitons using magnetic field where Zeeman interaction is utilized to overcome the intervalley exchange interaction $[75,76,77]$. In this work, we demonstrate that controllably charging an individual QD in a van der Waals heterostructure can also result in a partial recovery of the valley polarization. Pronounced spectral shifts in the voltage-dependent photoluminescence signal provide evidence of charging. The polarization of photons emitted by the TMDC QD further confirms the formation of trions. The observation, creation and voltage control of quantum confined trions in our device is the central result of this chapter.

\subsection{Device architecture}

Our device (see Fig. 6.1a), is a van der Waals heterostructure [44]. An optical micrograph of a typical three terminal device is presented in Fig. 6.1b. It consists of a tungsten diselenide ( $\mathrm{WSe}_{2}$ ) layer, which naturally hosts QD like emitters [74], sandwiched by insulating hexagonal boron nitride layers and capped on the top and bottom by few layer graphene flakes that serve as electrodes. We use 

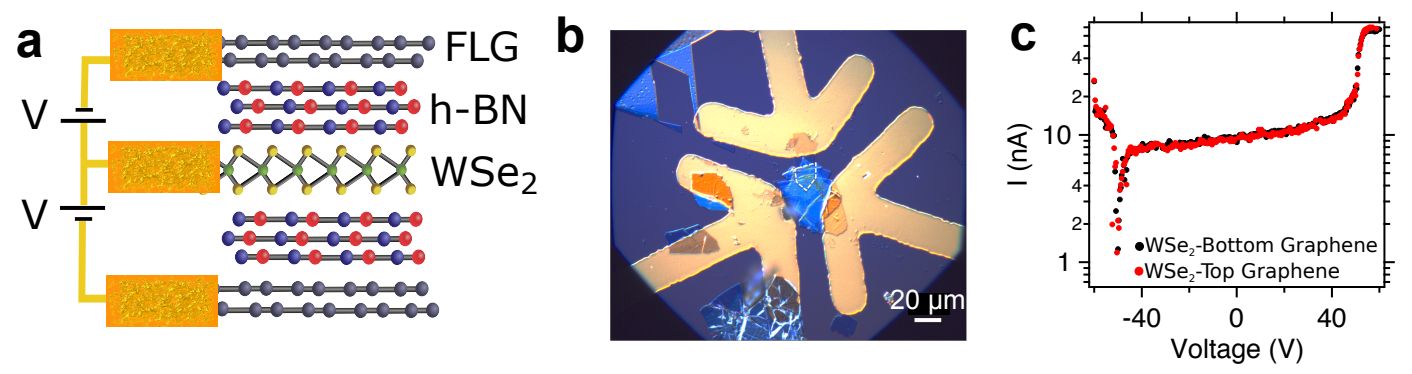

Figure 6.1: Device architecture: a. A schematic representation of the van der Waals heterostructure that creates a heterojunction device for electrostatic charging of $\mathrm{WSe}_{2}$ hosting quantum dots. FLG $=$ few layer graphene flake, $\mathrm{h}-\mathrm{BN}=$ hexagonal boron nitride. b. An optical micrograph of a three terminal device as illustrated in Fig. a. The heterostructure is outlined in white dotted line. c. Current (I) measured as a function of voltage (V) applied across the bottom graphene-WSe 2 (Black data) and top graphene- $\mathrm{WSe}_{2}$ (red data).

electrostatic doping through the top and bottom graphene electrode to generate charged 3D confined excitons. The current-voltage (IV) characteristics of the device is presented in Fig. 6.1c.

\subsection{Identification of neutral exciton, trion and biexciton}

As illustrated in Fig. 6.2b, dependent on the applied voltage, the QD stabilizes either a localized neutral exciton and biexciton or a negatively charged exciton a trion - excited state. To identify QD emission and characterize their charging state the sample is raster scanned to locate spectrally narrow $(100 \mu \mathrm{eV}-400 \mu \mathrm{eV})$ emission peaks red shifted from the delocalized neutral excitons in $\mathrm{WSe}_{2}$ that emit at $\sim 1.75 \mathrm{eV}$ [74]. After locating the emitter, the spectral content of the 

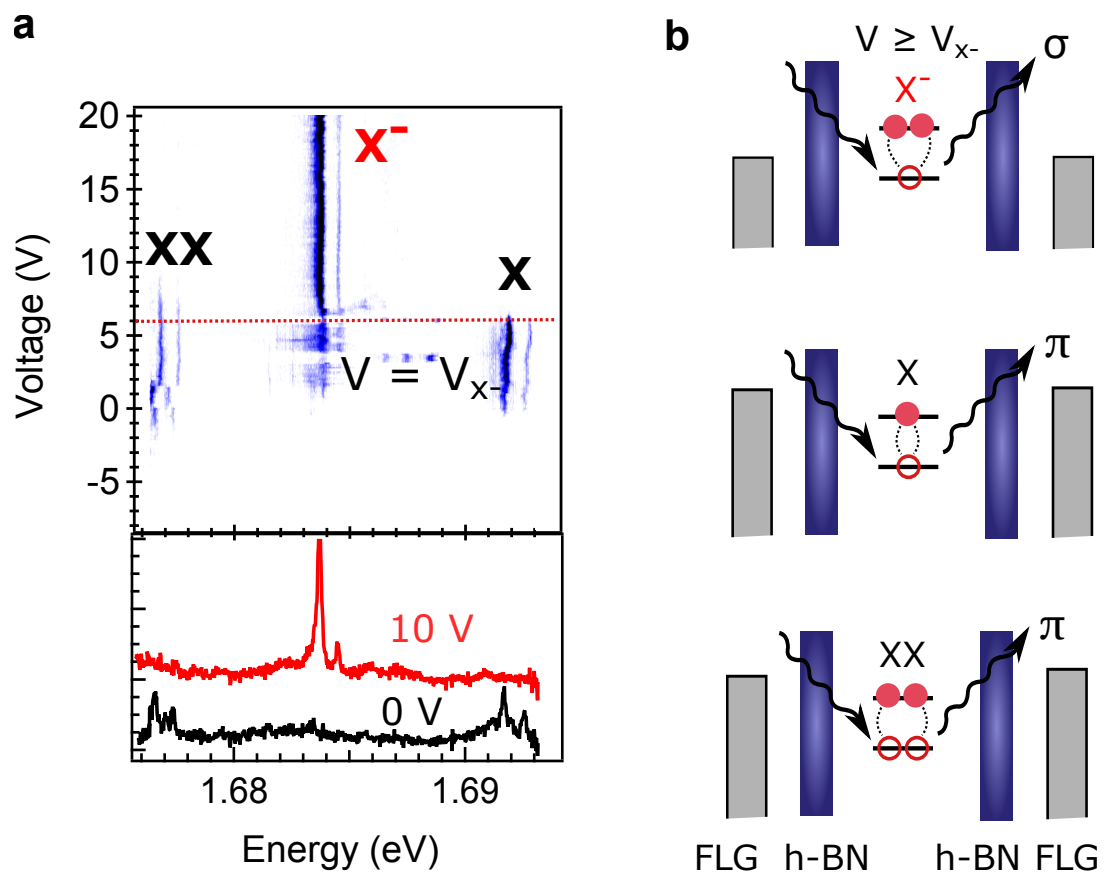

Figure 6.2: PL vs voltage: a. Voltage dependent photoluminescence demonstrating a distinct spectral jump between two emission lines. The higher voltage plateau above $\mathrm{V}_{X-}$ (indicated by dashed red line) originates from a localized trion state $\left(\mathrm{X}^{-}\right)$whereas the two lower voltage plateau are from the neutral exciton $(\mathrm{X})$ and biexciton (XX) emission. The line-cuts in the inset are taken above (10 V) and below $(5 \mathrm{~V}) \mathrm{V}_{X_{-}}$. b. Formation of the trion $\left(\mathrm{X}^{-}\right)$in the device by capture of an extra electron leads to a suppression of the intervalley mixing from the electron-hole exchange interaction giving rise to valley polarized emission (top). For neutral excitons $(\mathrm{X})$ the intervalley electron-hole exchange interaction mixes the two valley states giving rise to linearly polarized emission (middle) which is also inherited by the biexciton feature (XX, bottom). Figure adapted from [136] with permission.

PL as a function of voltage applied across the device is recorded. An exemplary data set that shows $\mathrm{QD}$ charging and photoluminescence $(\mathrm{PL})$ voltage plateaus 
for each emitter species is presented in Fig. 6.2a. The neutral exciton (X) and the biexciton (XX) share a common voltage plateau as expected. With increasing voltage, appearance of a singly charged trion $\left(\mathrm{X}^{-}\right)$is observed. The bottom panel of Fig. 6.2a are line-cuts at two different voltages also indicating the presence of valley mixed exciton states for the neutral exciton, biexciton and the trion, exhibited by the splitting in the emission lines commonly referred to as the fine structure splitting (FSS). We focus on the singly charged exciton for the rest of the manuscript. The observed behavior is characteristic of all emitters that have been measured. The PL intensity of both the exciton and the biexciton reduces as the trion appears, whose intensity increases with increasing voltage. The PL spectral jump and the distinct PL voltage plateaus are indications of trion formation and has been observed in similar diode based indium arsenide quantum dot devices $[114,145]$. From the PL map, the difference in the exciton and trion emission energy is around $9 \mathrm{meV}$ which is a measure of the trion's binding energy $\left(E_{X}-E_{X^{-}}\right)$. The average binding energy from the measured emitters is $8 \mathrm{meV}$ with a standard deviation of $4 \mathrm{meV}$. Binding energy of the charged complexes can fluctuate due to randomness of the environment [146].

\subsection{Power dependence of the voltage plateaus}

We further confirm the different excitonic complexes by measuring PL intensities as a function of excitation power. Figure 6.3a presents power dependent intensity of the exciton and biexciton line. The intensity of the biexciton feature increases faster as a function of power compared to the neutral exciton. Fitting these lines to a power law $\left(I=A+B \cdot I^{n}\right)$ yields a coefficient of $\mathrm{n}=0.64$ for the exciton and $\mathrm{n}=1.31$ for the biexciton confirming the sub and super-linear behavior as expected 

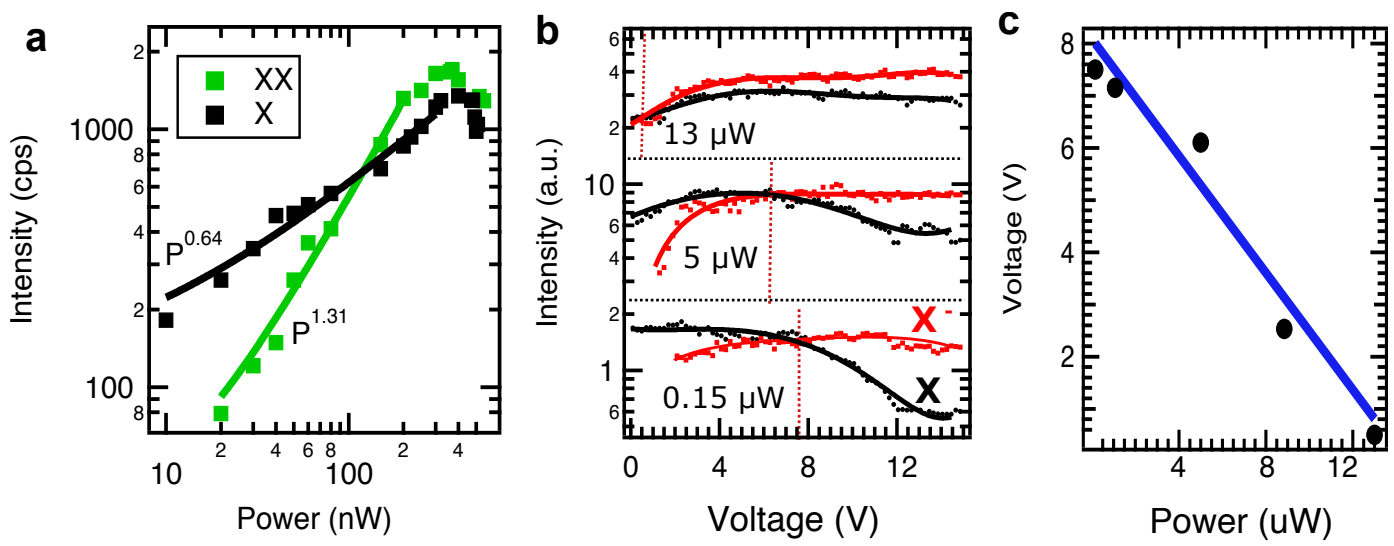

Figure 6.3: Photoluminescence analysis: a. Power dependent PL intensity for the exciton (X) and the biexciton (XX) lines, b. Power dependence of the exciton and trion voltage plateaus for 3 different powers. Starting from top: $13 \mu \mathrm{W}, 5$ $\mu \mathrm{W}$ and $0.15 \mu \mathrm{W}$. The red dotted vertical lines represent the voltage at which the trion to exciton crossover occurs at each power. c. Voltage threshold for exciton to trion crossover indicating a linear dependence (red dotted line) on excitation power. Figure adapted from [136] with permission.

from the exciton and biexciton respectively [98]. The influence of the laser power on the charging process is presented in Fig. 6.3b. PL voltage profiles for 3 different excitation powers is shown. As the pump power increases (from bottom to top panel in Fig. 6.3b), the photogeneration of negative trions becomes more efficient. This also results in reduction of the threshold voltage required for the charging process in response to the additional built-in field caused by excess photogenerated carriers (Fig. 6.3c) [147]. In our data, this is exhibited by the voltage at which a crossover from exciton to trion occurs. The lower effective mass of electrons in $\mathrm{WSe}_{2}$ [148] results in a preference for photogenerated negatively charged trion formation [149]. 


\subsection{Polarization dependence}
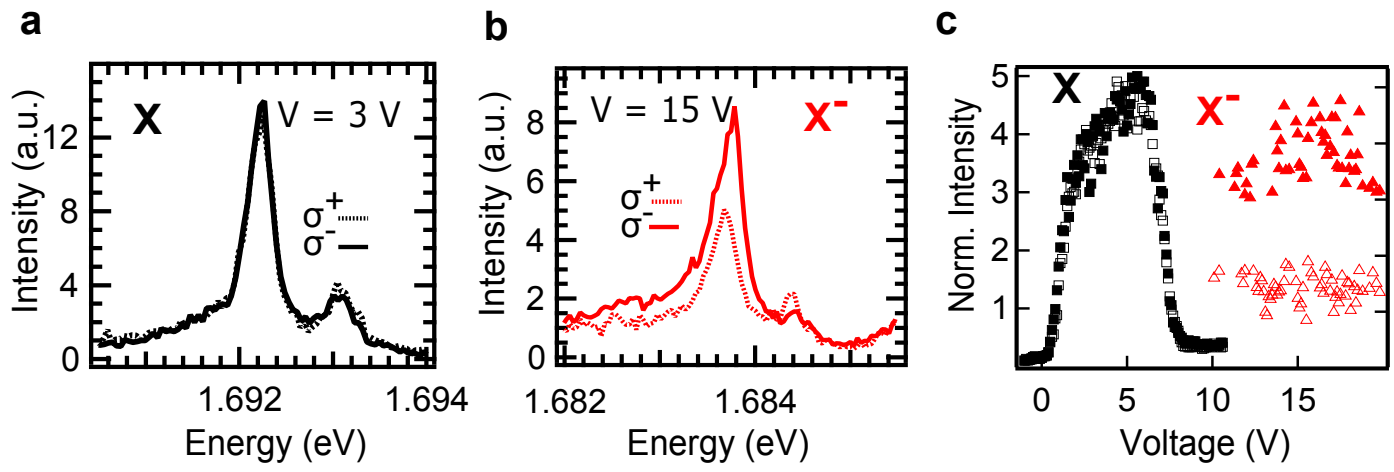

Figure 6.4: Polarization resolved PL : a, b. $\sigma^{-}$and $\sigma^{+}$emission spectra of the exciton (trion) at $3 \mathrm{~V}(15 \mathrm{~V})$. The solid (dotted) line represents $\sigma^{-}\left(\sigma^{+}\right)$detection. c. Evolution of the emission intensity in the circular detection basis as a function of voltage mapped for the lower energy peak of the exciton (in black circles) and trion (red triangle). Solid (open) legends represent $\sigma^{-}\left(\sigma^{+}\right)$polarized emission intensity. Excitation laser is $\sigma^{-}$polarized. Figure adapted from [136] with permission.

The polarization of the emitted photons also carries information regarding the QD's charging state. Polarization resolved measurements are performed using the microscope setup described in chapter 3, section 3.3.2. For polarization resolved measurements, the QD is excited with circularly polarized light and the $\sigma^{-}$and $\sigma^{+}$polarized PL is analyzed in Fig.6.4a and b. The polarization is studied at 3V and at $15 \mathrm{~V}$. The polarization resolved spectra indicate the split states of the trion preserves the polarization selection rules [106] (Fig. 6.4b) where the lower energy is $\sigma^{-}$polarized whereas the higher energy peak is $\sigma^{+}$polarized. However the exciton peaks do not prefer any circular polarization states exhibiting a predominantly linear polarization (Fig. 6.4a). The previous is examined as a function of applied voltage in Fig. 6.4c. In the data, the solid circles (triangles) correspond to 
intensity of $\sigma^{-}$emission for the lower energy peak of the exciton (trion) whereas the open circles (triangles) correspond to $\sigma^{+}$emission. Throughout the charging plateau, the studied trion peak preserves the $\sigma^{-}$polarization. The different charge configurations of the excited state provides a window into understanding the observed polarization contrast. In Fig. 6.5a, the DoCP is plotted for each of the exciton and trion voltage plateaus. For the neutral exciton (X, black circles), the DoCP is negligible. When the ground state $(|0\rangle)$ of a localized spin-valley emitter under the influence of anisotropic intervalley electron-hole exchange interaction is optically excited, an exciton with spin-valley mixed state is formed represented by $|+\rangle$ and $|-\rangle$. In this case, electron-hole exchange rate is comparable to or faster the radiative recombination rate of the individual valley exciton (as observed for case of the delocalized excitons [69]) giving rise to a mixture of $\sigma^{-}$and $\sigma^{+}$state. Next, we consider the trion that exhibits a fixed degree of circular polarization and averages at 44 percent as indicated by the red horizontal line (Fig. 6.5a). Nonzero DoCP throughout the trion voltage plateau is not unexpected as even the delocalized valley trions have a slower depolarization rate than the valley excitons [69]. For the case of these localized emitters, the finite DoCP across the plateau is due to a reduction of the electron-hole exchange interaction due to addition of an extra electron which is also confirmed by a reduction of the FSS when compared to the neutral exciton (Fig. 6.5b) The FSS and DoCP remains constant along the charging plateau of both the exciton and trion.

To understand the possible valley depolarization mechanisms for the trion, consider the ground state which is occupied by an extra electron $(|e\rangle)$. Optical excitation generates a localized trion along with a fine structure splitting. However now, there is a reduction in the electron-hole exchange interaction $\left(\Delta_{e-h}\right)$ compared to the neutral exciton due to the addition of extra electron (Fig. 6.5c). 
In InAs quantum dots, addition of an extra electron has shown to completely suppress the fine structure splitting due to the formation of a spin singlet state $[105,150]$. However, complete reduction of the intervalley exchange interaction is not observed for the case of the localized trions in these emitters. Prior studies on the delocalized trion states in monolayer $\mathrm{WSe}_{2}$ have confirmed that exchange interactions can also affect trion. A fine structure splitting results from electronhole and electron-electron interactions which splits the intervalley and intravalley trion states $[69,151,152]$. Depolarization in the trion can take place by exchange interaction (intervalley trion) or by a combination of exchange along with single particle spin flip (intra-valley trion) [69]. Spin flip processes are generally slower reducing the net depolarization rate. However, unlike the spin singlet states of the charged excitons in indium arsenide quantum dots $[105,150]$, the FSS of the 3D confined neutral exciton is at least an order of magnitude higher than the InAs quantum dots manifesting the strong exchange interaction energies in these emitters with reduced symmetry. As a result, the intervalley exchange interaction also influences the trion state preventing singlet formation. Alongside, in an earlier study it has been described that charging a quantum dot with a single electron may not guarantee complete suppression of the electron-hole exchange interaction [105]. This is possible for the case of quantum dots with shallow confinement where redistribution of wave function of the electron pair can lead to non-zero local spin density for the excited trion state. The previous carrier redistribution mitigating singlet formation is likely as the large FSS in the confined neutral excitons the result of strong coulomb interactions inherited from the monolayer TMDCs. 


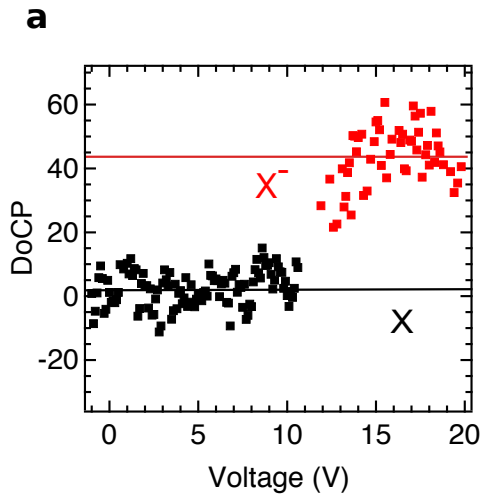

b
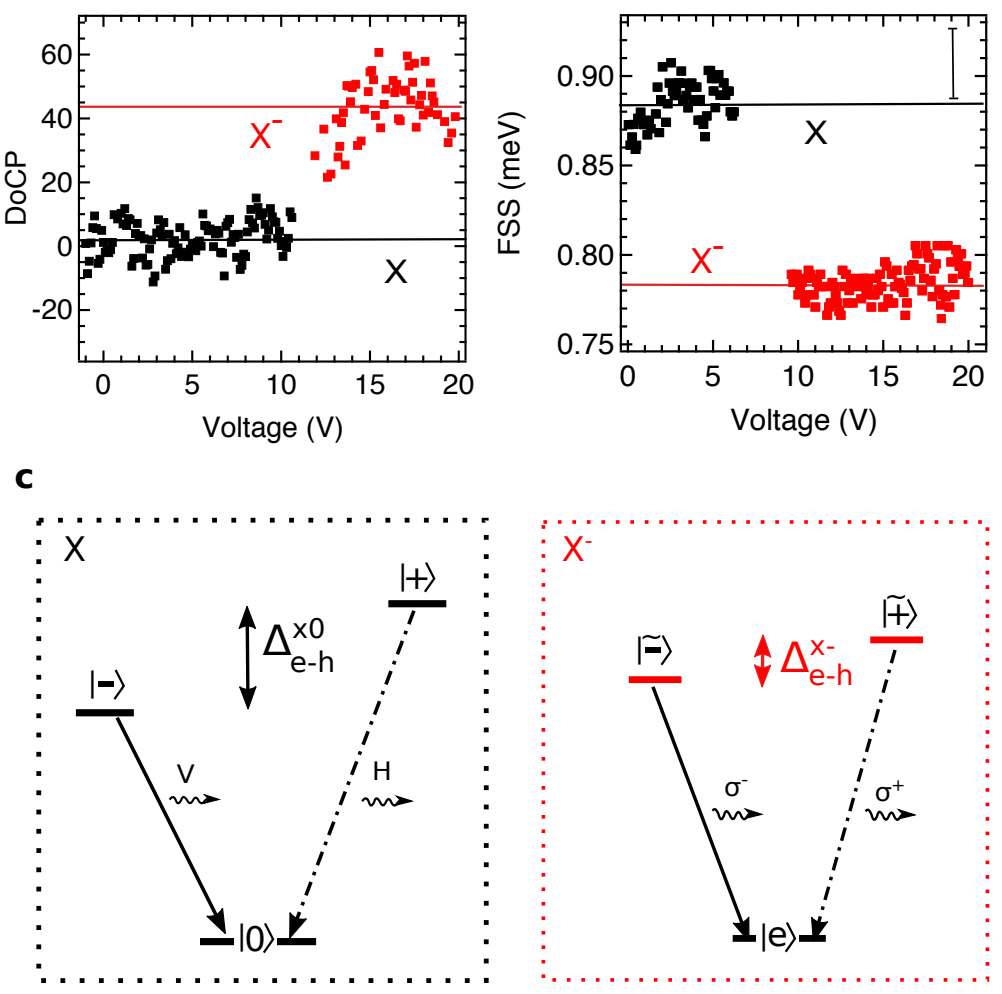

Figure 6.5: Valley polarization vs electric field: a. Degree of circular polarization of neutral exciton (X, black) and trion $\left(\mathrm{X}^{-}, \mathrm{red}\right)$ as a function of voltage. A scatter in the DoCP is due to fluctuations in intensity due to local environment of the emitter. b. Fine structure splitting as a function of voltage for the observed neutral exciton (black) and trions (red) generated from fit to Lorentzian function. Error bar indicates the resolution of the spectrometer. c. Schematics of energy levels of the neutral exciton and trion emission with fine structure splitting $\Delta_{e-h}^{x 0}$ and $\Delta_{e-h}^{x-}$ respectively due to electron-hole exchange interaction in an anisotropic potential. X represents the neutral exciton states with linearly polarized emission from the split states represented by $|-\rangle$ and $|+\rangle$. $\mathrm{X}^{-}$represents the trion states with an extra electron $(e\rangle)$ occupying the ground state causing a reduction in the electron-hole exchange interaction $\Delta_{e-h}^{x-}$ and circularly polarized split states. Figure adapted from [136] with permission. 


\subsection{Summary}

In conclusion, we have demonstrated the controlled charging of TMDC-QDs in a van der Waals heterostructure. A reduction in the fine structure splitting along with a finite degree of circular polarization is observed for the case of the charged emitter. Stable, and optically accessible individual ground state spins are critical for the application of van der Waals heterostructure based QDs in quantum photonics and metrology. Understanding the interplay between both valley and spin lifetimes and coherences is a natural next step. We also expect coherent optical control of the spin ground states to be possible via the excited state trions not only providing a pathway for all-optical information processing [153, 154], but also opening new directions in solid-state quantum optics studies. 


\section{Chapter 7}

\section{Integration of $2 \mathrm{D}$ materials}

\section{with plasmonic and photonic}

\section{devices}

In this chapter, we will study integration of $2 \mathrm{D}$ materials with photonic and plasmonic nanostructures.

\subsection{Optical antenna enhanced graphene photode- tector}

In the first section of this chapter, we will discuss the integration of an individual, metal-based, plasmonic nano-antenna on a graphene photodetector [155]. The device exhibits an electromagnetic resonance at a wavelength of $580 \mathrm{~nm}$ with well-defined polarization sensitivity. With no applied bias voltage, this antennacoupled graphene photodetector features a responsivity of $\sim 17 \mathrm{nA} / \mu \mathrm{W}$, which is four orders-of-magnitude higher than previously studied single antenna-enhanced

detectors. Finally, we measure a signal-to-noise ratio of $\sim 200$ in a one-Hertz 
bandwidth, with an average photocurrent value of $1.2 \mathrm{nA}$.

\subsubsection{Introduction}

Graphene, a single layer of carbon atoms, has become increasingly popular owing to its unique electrical, mechanical and optical properties [156, 157, 158, 159]. Its broadband absorption and ultrafast transport makes it an excellent candidate for optoelectronic devices. However, its low absorption, lack of intrinsic band gap and low quantum yield inhibits its performance as a photodetector [160]. It is well known that plasmonic based metallic nano-antennas can improve the light harvesting properties of weak light absorbers [161], so, such devices are a natural candidate to improve graphene's optical response.

When a metal nanoparticle (NP) interacts with light, the incident electric field can drive the conduction electrons to collective oscillations known as localized surface plasmon resonance (LSPR). This resonance alters the incident radiation pattern drastically and leads to effects such as sub-wavelength localization of electromagnetic energy, formation of high intensity hot spots or anisotropic light scattering angular spectra $[162,163]$. These effects have already been utilized for modifying the radiative and non-radiative properties of emitters like NP coupled molecules, atoms or quantum dots [164, 165]. Further, metal nanoparticles have been extensively integrated with two-dimensional, atomically-thin photodetectors in forms like colloidal particles[166, 167] and plasmonic-antenna arrays [168, 169, $170,171]$.

Antenna integrated graphene photodetectors have been demonstrated to work in the visible-near infrared (IR) [168], the mid-IR [172] and the far-IR [173, 174]. Most of them show the collective photoresponse from multiple antennas on large area CVD graphene photodetectors. In contrast to previous work, we report on 
photocurrent enhancement from a lithographically fabricated single nano-antenna with dimensions smaller $\sim \lambda / 2$ of light on an exfoliated graphene photodetector. The small detection area has application in nanoscale optoelectronic devices. The previous studies of nano-antenna arrays on graphene motivate the investigation of how an individual antenna can enhance graphene's optical response. Different in our device is that the antenna not only localizes and enhances the illuminating electromagnetic field, but it also provides the electrical contacts for extracting light-induced current. Plasmon resonances in individual nano-gap electrodes have also been explored using graphene constrictions [175], but patterning graphene may open up a band gap [176] which could limit its spectral responsivity. Additionally, by rationally designing and fabricating a well-defined antenna structure we are able to control the full electromagnetic response of the antenna.

\subsubsection{Device structure}

An illustration of an antenna-on-graphene device with an external electrical circuit for photo-current detection is presented in Fig. 7.1a. We exfoliate graphene from bulk graphite crystals by micromechanical cleavage using adhesive tape [34]. The substrate consists of a doped silicon wafer with a $300 \mathrm{~nm}$ thermally-grown oxide layer that facilitates single-layer graphene identification under an optical microscope $[34,156,18]$. Figure $7.1 \mathrm{~b}$ presents the false color scanning electron microscope (SEM) image of a typical graphene device integrated with an individual nano-antenna. The top inset of Fig. 7.1b shows a magnified SEM image of a fabricated antenna. The antenna is connected to the larger source-drain metal contacts via fingers from the antenna. This enables us to directly read out the graphene electrical signal from light that is localized by the antenna. The device was fabricated using electron beam lithography followed by electron beam 
metallization with $\mathrm{Cr}(1 \mathrm{~nm}) / \mathrm{Au}(29 \mathrm{~nm})$. The electrodes were wire-bonded and connected to external electronics. The position and relative intensity of the $\mathrm{G}$ and 2D peak in Raman spectra (bottom inset, Fig. 7.1b) confirms the flake is singlelayer graphene. Also, no disorder induced D band was observed in the Raman spectra indicating that the flake was not damaged during the fabrication process. Interestingly, we observe an increase in the intensity of the Raman modes in the antenna-laden graphene region (green curve, bottom inset, Fig. 7.1b) with respect to the antenna-free region (black curve) for 532-nm laser illumination [168]. However, this near-field enhancement is not significantly large due to off-plasmon resonance excitation.

\subsubsection{Simulation and measurements}

Finite difference time domain (FDTD) (Lumerical software) simulations were used to design the nano-antenna. The length $(\mathrm{h})$ and width $(\mathrm{w})$ of the antenna were 80 $\mathrm{nm}$ and $70 \mathrm{~nm}$ respectively with $40 \mathrm{~nm}$ wide fingers contacting both sides of the antenna (see inset of Fig. 7.2a for the antenna geometry). FDTD simulated scattering spectra of the fabricated antenna shows a resonance around $560 \mathrm{~nm}$ with a linearly polarized plane wave excitation source. Inset of Fig. 7.2a presents the simulated electromagnetic field distribution of the antenna excited with 532-nm source in perpendicular polarization (along direction of blue arrow). The antenna is outlined in black dashed line as a guide to the eye. The enhancement is maximum around the four corners of the antenna. The resonance is suppressed when the source is polarized parallel to the antenna long-axis. The antenna was designed to exhibit this unconventional polarization response. The main motivation was to mitigate against any possible resonance damping effects that could result from the presence of the metallic fingers connecting the antenna to the electrodes. The 

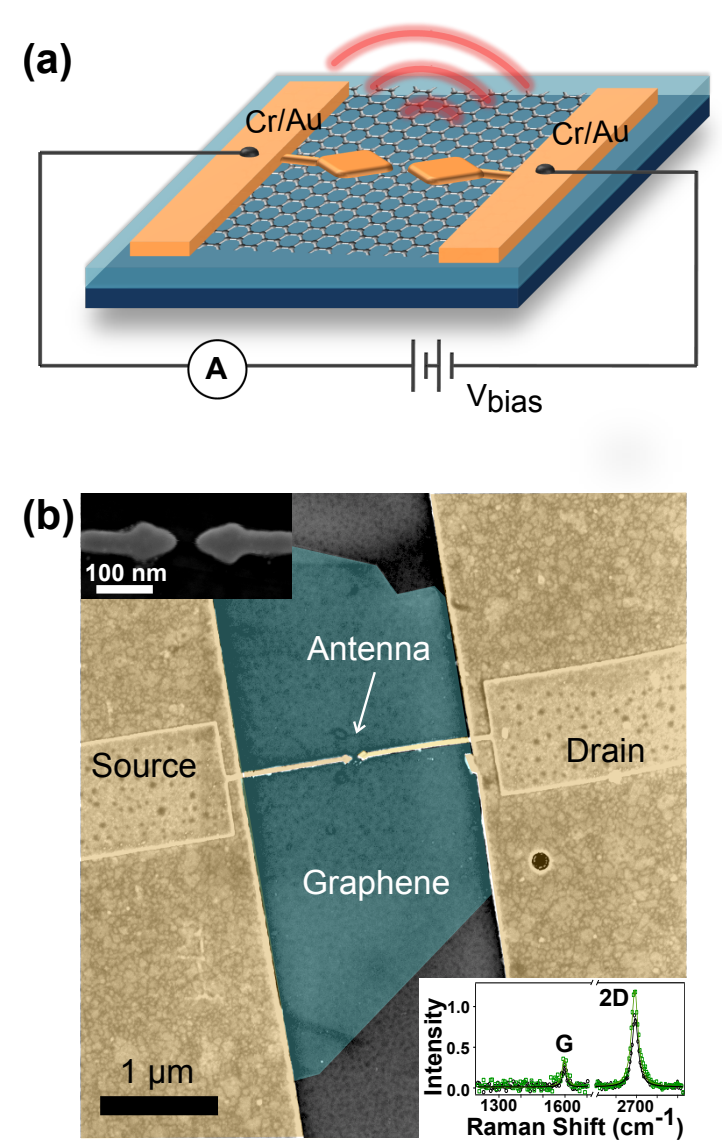

Figure 7.1: Device schematic and SEM micrograph: a. Illustration showing an antenna-on-graphene device connected to external electronics. b. False color scanning electron micrograph of a representative antenna-on-graphene device. Inset (top left): zoomed in image of the antenna. Inset (bottom right): Raman spectra of antenna-laden (green) and antenna-free (black) regions of graphene. Solid lines are Lorentzian fits to the data. Figure adapted from [155] with permission.

broad scattering spectrum exhibited by the antenna is characteristic of a plasmon mediated electromagnetic resonance and is due to damping experienced by charge oscillation of the LSPR when scattering with the nano-structure boundary [177].

To characterize the optical response of our fabricated antenna the devices were illuminated with a 532-nm laser, in a home-built inverted confocal microscope, us- 

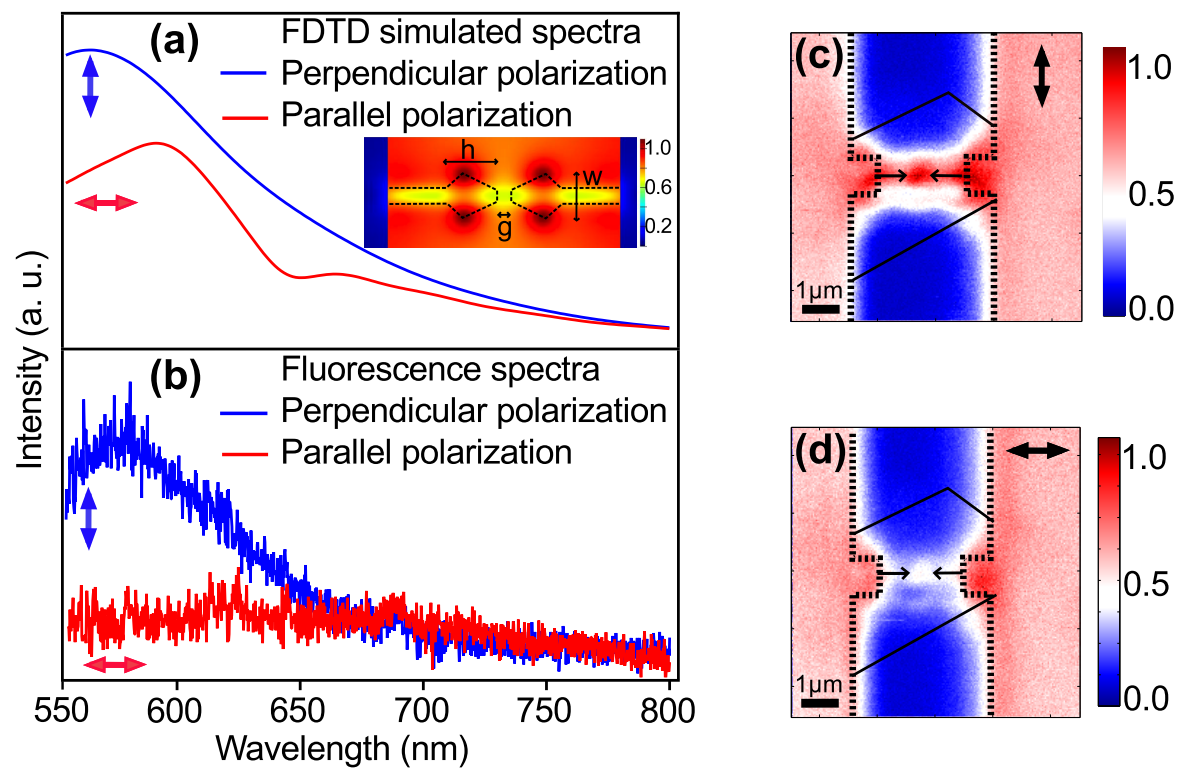

Figure 7.2: Simulation and measurements: a. Normalized FDTD simulated spectra and b. measured fluorescence spectra showing polarization dependence of gold luminescence of the antenna in perpendicular (blue) and parallel (red) direction of excitation laser. Backgrounds are subtracted. Excitation wavelength, $\lambda=532$ $\mathrm{nm} ;$ power $=60 \mu \mathrm{W}$. Inset of (a) shows the simulated electromagnetic field distribution of the antenna. Normalized scanning fluorescence map of the device with laser excitation polarization c. perpendicular and d. parallel to the antenna. Dotted lines trace the outline of the contact electrodes and the region within the arrows indicates the enhancement due to the antenna for both (c) and (d). The solid black line outlines the graphene flake between the contact electrodes. Arrows denote the polarization of incident laser. Figure adapted from [155] with permission.

ing a microscope objective with a numerical aperture (NA) of 0.75 . Figure $7.2 \mathrm{~b}$ presents the measured fluorescence spectrum. The perpendicular and parallel polarization correspond to both the polarization orientation of the illuminating laser and the polarization orientation of the fluorescence spectrum collection channel. 
The fluorescence spectrum confirms that the fabricated nano-antennas exhibits the designed electromagnetic response; they have a plasmon resonance within the visible wavelength range $(\sim 580 \mathrm{~nm})$ and in the polarization direction perpendicular to the antenna long axis (Fig. 7.2b). The difference in resonance peak of our designed structure (inset of Fig. 7.2b) from FDTD simulated structure is due to fabrication imperfections such as surface roughness and size mismatch [178]. In addition to the antenna spectral response, we also acquired scanning fluorescence images of the device. The sample was mounted on a translational stage (Mad City Labs, Inc.) that was raster scanned through the focused laser and photo-counts were recorded with an avalanche photodiode. Evident in the images presented in Fig. 7.2c and Fig. 7.2d is that the maximum fluorescence enhancement is obtained when the excitation and collection polarization orientation is perpendicular to the antenna long axis (the long axis is parallel to the antenna gap). 

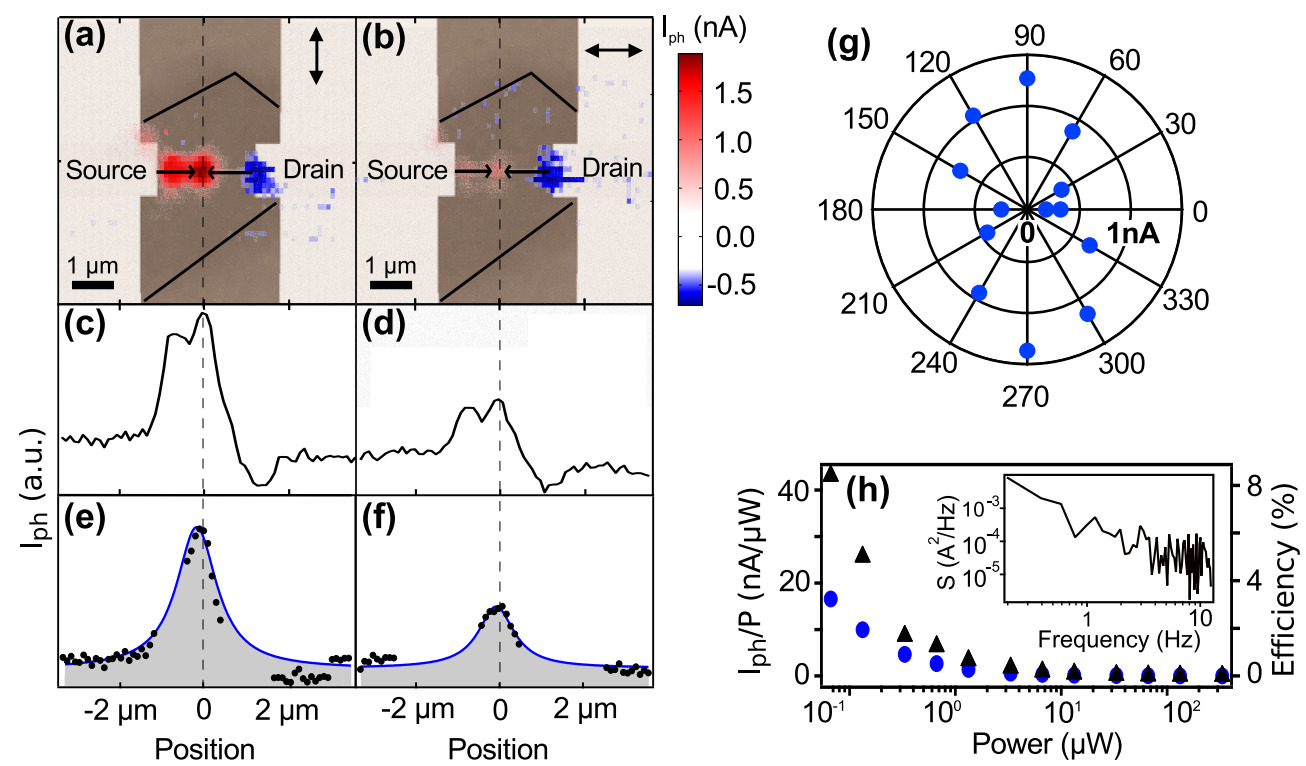

Figure 7.3: Polarization and photoresponse: a, b. Scanning photocurrent image superimposed on a device design with excitation polarization (a) perpendicular and (b) parallel to the antenna's long axis. Color scale denotes photocurrent in nA units. Arrows denote polarization of incident laser. c, d. Horizontal slices of photocurrent through the antenna in panel (a) and panel (b) respectively showing a symmetric current response due to antenna superimposed with an antisymmetric response from photodetection at graphene-metal junction. e, f. Contribution in photocurrent from antenna-on-graphene region (area indicated within black arrows in photocurrent map) which is extracted from panel (c), (d) by subtracting the antisymmetric current from either side of symmetric antenna response. $\mathrm{g}$. Polar graph showing polarization anisotropy of photocurrent of a typical device. The radius in the polar graph has a photocurrent magnitude of $1.5 \mathrm{nA}$. Current is maximum for vertical polarization of laser $\left(90^{\circ}\right)$ and minimum for horizontal polarization $\left(0^{\circ}\right)$. h. Photoresponse $\left(\mathrm{I}_{p h} / \mathrm{P}\right)$ and external quantum efficiency (Efficiency) as a function of power showing sub-linear dependence of response for both zero bias (blue circles) and at a bias of $0.5 \mathrm{mV}$ (black triangle). Inset shows the noise power density of the dark current from the detector measured at zero bias. Figure adapted from [155] with permission. 
To investigate the photoresponse of the device we use a home-built, room temperature, scanning photocurrent (SPC) microscopy setup. The device was illuminated with a laser at excitation wavelength of $635 \mathrm{~nm}$ (laser power $=25$ $\mu \mathrm{W}, \mathrm{NA}=0.8)$. The sample was raster scanned and a current value was recorded using a Keithley 2400 sourcemeter. All photocurrent measurements were taken at zero bias to eliminate background current and obtain a gain-free enhancement in photocurrent resulting from the antenna [179]. In Fig. 7.3a, a photocurrent map is shown for excitation laser polarized perpendicular to the antenna's long axis. In the SPC map, photocurrent is present in two principal regions: at the source-drain contacts with graphene and in the vicinity of the antenna-on-graphene. Local electric fields are induced at the graphene-electrode junction [180, 181]. When light is incident at these junctions, electron-hole pairs are created which generate a photocurrent even at zero bias [182] as a result of structural asymmetry in the device. Figure $7.3 \mathrm{~b}$ is a second photocurrent map of the same device, exhibiting diminished photocurrent in the antenna region, for laser excitation polarization parallel to the antenna's long axis.

Figure 7.3c (Fig. 7.3d) presents a horizontal line-cut of the current along the antenna in the SPC map from panel (a) [panel (b)] of Fig. 7.3. The previous linecuts demonstrate that the current response is antisymmetric at opposing contacts, superimposed with a symmetric plasmon mediated current peak centered around the gap of the antenna. Such antisymmetric photocurrent as a function of position has been previously reported for metal contacts on graphene due to contributions from a thermoelectric effect superimposed with a photovoltaic effect $[180,183]$. In Fig. 7.3e and $\mathrm{f}$ the antenna response is extracted from the line-cut in panel (c) and (d) by subtracting the symmetric photocurrent response from either end of the antenna response and fit with a symmetric Lorentzian function. Comparing the 
fluorescence maps (Fig. 7.2a and b) with the photocurrent maps (Fig. 7.3a and b), we observe that the maximum polarization sensitivity of photocurrent arises from the same antenna region as obtained from the fluorescence map, which further confirms the plasmonic enhancement from the antenna-on-graphene region.

The polar plot in Fig. 7.3g shows the polarization dependence of the photoresponse from the nano-gap antenna on graphene region, which is consistent with the antenna design and fluorescence spectrum measurements. The polarization contrast $\left(\left|I_{\max }\right|-\left|I_{\min }\right|\right) /\left(\left|I_{\max }\right|+\left|I_{\min }\right|\right)$ is $76 \%$. Although the photoresponse is low compared to previous studies using arrays of antenna on graphene [168, 170], we are able to extract a maximum photoresponse of $\sim 17 \mathrm{nA} / \mu \mathrm{W}$ at zero electrical bias (Fig. 7.3h) from a typical antenna-on-graphene device when illuminated with $66 \mathrm{nW}$ of optical power. This is four orders-of-magnitude higher than previous reports on similar single antenna graphene photodetectors [175]. We attribute the increase in photoresponse to light concentration mediated by the antenna and material quality since our fabrication process does not directly pattern the graphene flake. Furthermore, the quantum efficiency of this device is calculated to be $3.2 \%$ under zero bias and $8.4 \%$ for a bias of $0.5 \mathrm{mV}$ (Fig. 7.3h). We observe that if the quantum efficiency was limited only by graphene absorption then the maximum value under zero bias would be $2.3 \%$. The increase of quantum efficiency (= number of electron-hole pairs detected in the photocurrent/number of incident photons) is further evidence of the antenna enhancing the detector's responsivity. Finally, we determine that our antenna-enhanced detector exhibits a signal-tonoise ratio of $\sim 200$ in a one Hertz bandwidth with an average photocurrent value of $1.23 \mathrm{nA}$ measured from a time trace of photocurrent in 40-ms bins (not shown). We also find a NEP of our detector by taking the noise power spectrum of the background current trace (Inset of Fig. $7.3 \mathrm{~h}$ ) from which we obtain $\mathrm{S}(\mathrm{f}=1 \mathrm{~Hz})=$ 
$3.1 \times 10^{-4} \mathrm{nA}^{2} / \mathrm{Hz}$. This determines the detector NEP to be $1.1 \times 10^{-9} \mathrm{~W} / \sqrt{\mathrm{Hz}}$ at zero bias.

\subsubsection{Summary}

In conclusion, we have integrated a single optical antenna on a single layer of graphene. The antenna concentrates the illuminating electromagnetic field and also serves as the electrical contacts used for extracting the generated photocurrent. Our approach to enhance the light harvesting ability of a graphene photodetector resulted in responsivities that were nearly four orders-of-magnitude larger than previous single antenna devices. It is expected similar approaches based on optical antennas will also be useful in tailoring the optical response of atomically-thin semiconductors. 


\subsection{Direct on-chip plasmon detection with mono- layer $\mathrm{MoS}_{2}$}

In the following section, we will demonstrate an atomically-thin molybdenum disulfide-based photodetector in a photonic integrated circuit and show that ultrathin materials can be used for the direct measurement of optical plasmons in a gain-free configuration [184]. We have found that atomically-thin materials are robust for near-field photodetection and for coupling to optical plasmons. This work connects the fields of nanophotonics, nanoplasmonics, and atomically-thin materials and provides a stepping stone for more complex nanoscale circuitry.

\subsubsection{Introduction}

The drive to make photonic devices more efficient within a smaller footprint has made the field of nanophotonics desirable for applications in integrated optoelectronics [185, 186, 187]. However, reducing the dimensions of these devices below the optical diffraction limit is challenging. Surface plasmon polaritons [188, 163, 189] are natural candidates for subwavelength electromagnetic energy confinement. They are evanescently-confined electromagnetic excitations that can propagate along a metal-dielectric interface. In addition to subwavelength light guiding, nanophotonic sources and nano-photodetectors are also desirable [190, 191, 179, 192]. The surface plasmon polaritons in silver (Ag) nanowires have been heavily studied in regard to guiding light beyond the diffraction limit [193, 194, 195]. Along with that the nanowires have demonstrated efficient cou-

pling with a variety of nanostructures $[196,197,198,199]$. Some of the exemplary optoelectronic devices are integrated graphene and germanium based modulators [200] and transistors [179] respectively. In this section, we demonstrate a device 
where Ag nanowire has been successfully integrated with a monolayer TMDC, $\mathrm{MoS}_{2}$. This device has the capability to electrically detect the near field optical plasmons propagating in the silver nanowire. We find the maximum plasmon-tocharge conversion efficiency and plasmon reponsivities to be 0.5 and $250 \mathrm{~mA} / \mathrm{W}$, respectively. Although the photoresponsivity is lower than previously-studied ultrasensitive $\mathrm{MoS}_{2}$ detectors [201], it is considerably larger than comparable untreated $\mathrm{MoS}_{2}$ devices $[202,203]$. Finally, we examine the device's spectral response demonstrating that the measured current is limited either by the nanowire transmission or the $\mathrm{MoS}_{2}$ absorption.

\subsubsection{Device geometry and fabrication}
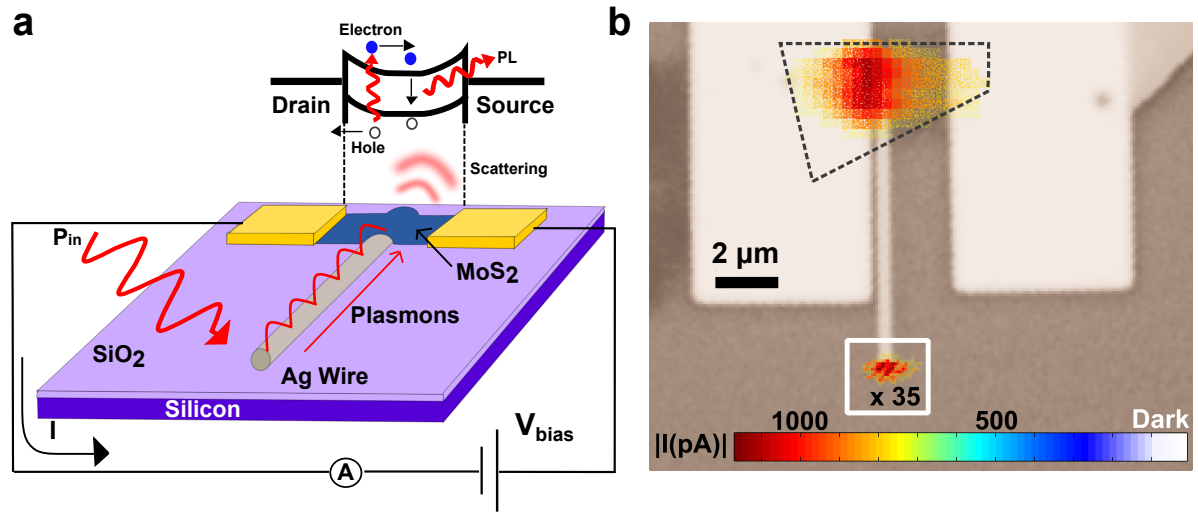

Figure 7.4: Scanning photocurrent map: a. Schematic of the $\mathrm{MoS}_{2} /$ nanowire detector. Inset: Band diagram showing pathways after electron-hole pair creation. b. Scanning photocurrent image of Device 1 at $\mathrm{V}_{\text {bias }}=0 \mathrm{mV}$, overlaid with an optical micrograph of the device. The flake is outlined with a dotted line. The region in the solid white box is measured with higher spatial resolution and the current value is multiplied by a factor of 35 . Excitation power $=25 \mu \mathrm{W}, \lambda=635$ nm. Figure adapted from [184] with permission. 
An illustration of the fabricated $\mathrm{MoS}_{2} / \mathrm{Ag}$ nanowire device is presented in Figure 7.4a. The nanowire is partially covered with a single-layer $\mathrm{MoS}_{2}$ flake which had been confirmed by PL and Raman measurements (Refer to section 2.1.1). Devices used in this study (referred to as Device 1 and Device 2) were fabricated through multiple steps using a poly(methyl methacrylate) (PMMA) liftoff technique, with several additional steps as described previously [197]. A brief description of the fabrication technique is provided. First, a solution of silver nanowires (Nanostructured and Amorphous Materials, Inc.) with $386 \mathrm{~nm}$ average diameter and $8.6 \mu \mathrm{m}$ average length was diluted and dispersed onto a glass coverslip. The coverslip was then attached to the bottom of a petri dish, which was then filled with water. Once a monolayer $\mathrm{MoS}_{2}$ flake was exfoliated and identified on a separate silicon with oxide substrate $\left(\mathrm{Si} / \mathrm{SiO}_{2}\right)$, we lifted the flake off by coating the sample in PMMA and immersing it in a hot sodium hydroxide solution. The PMMA film was then transferred onto the petri dish water bath, and the film was transferred onto a desired wire with the aid of an inverted microscope. After the PMMA was dissolved in acetone, the liftoff was then completed a second time, but the PMMA film was scooped onto an $\mathrm{Si} / \mathrm{SiO}_{2}$ substrate that was marked for alignment. Source-drain contacts were patterned using electron beam lithography and subsequent evaporation of a chromium base layer and gold. This enabled both photocurrent measurement and voltage biasing. A photon incident on the bare end of the nanowire (i.e., not covered with the TMDC flake) generates a plasmon via scattering $[193,195]$. We will study the plasmons that propagate along the nanowire and induce a current in $\mathrm{MoS}_{2}$ (referred to as the plasmon current, $I_{\text {plas }}$ ). 


\subsubsection{Scanning photocurrent microscopy}

Scanning photocurrent microscopy was used to investigate the devices. The samples were characterized with an inverted microscope equipped with an air objective (numerical aperture $=0.80)$. A nanopositioning stage (Mad City Labs, Inc.) was used to scan and position the sample. Excitation polarization was controlled by a half-wave plate and all electrical characterization was done via a Keithley 2601 sourcemeter. Scanning photocurrent images and polarization and responsivity data were obtained using a diode laser (ThorLabs, $\lambda=635 \mathrm{~nm}$ ). Spectral characterization was done using a Koheras SuperK supercontinuum laser and bandpass filtering.

Figure $7.4 \mathrm{~b}$ presents a typical position-resolved photocurrent map, overlaid on an optical microscope image, from Device 1 under no external voltage bias. The single-layer $\mathrm{MoS}_{2}$ flake is represented by the dashed box. Apparent in the photocurrent map is direct photocurrent generation in the $\mathrm{MoS}_{2}$ flake [202] and photocurrent when the far-field photons excite Ag nanowire optical plasmons at the uncovered end of the nanowire (solid white box). We will refer to the latter photocurrent as 'plasmon current'. Surface plasmons in silver nanowires can only be excited at irregularities in the wire or at the either ends of the nanowire to account for the wavevector mismatch between incoming photons and surface plasmons, which have larger wavevector than incoming photons. A plasmon current is observed even for the gain free configuration of the device (zero bias voltage). Referring to the inset of Fig. 7.4a, regions in the vicinity of the metal-MoS ${ }_{2}$ contact exhibit band bending inducing local electric fields [203] that are able to separate plasmon generated electron-hole pairs. In this simple illustration, the nanowire is ignored. In the following, we explore the optical and electrical properties of this optical plasmon induced $\mathrm{MoS}_{2}$ photocurrent. 

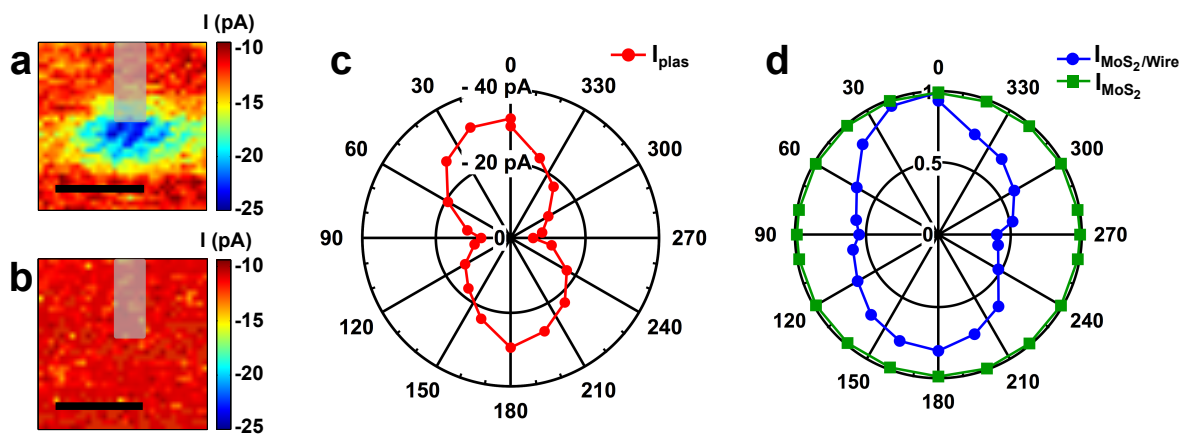

Figure 7.5: Polarization dependent photocurrent: a,b. Scanning photocurrent image of uncovered end of Ag wire of Device 1 (white box in Fig. 7.4b at $\mathrm{V}_{\text {bias }}=$ $0 \mathrm{mV}$ for laser excitation parallel (a) and perpendicular (b) with respect to the wire axis. The scale bars are $1 \mu \mathrm{m}$. The gray rectangle indicates the nanowire location. c. Plasmon current $\left(I_{\text {plas }}\right)$ as a function of laser excitation polarization. d. Normalized photocurrent for the $\mathrm{MoS}_{2} /$ nanowire interface (blue circles) and $\mathrm{MoS}_{2}$ alone (green squares) as a function of laser excitation polarization. For all data, $\lambda=635 \mathrm{~nm}$, power $=25 \mu \mathrm{W}$. Figure adapted from [184] with permission.

The plasmon current was further explored using polarization dependent excitation. The photocurrent map from the uncovered end of the nanowire (solid white box in Fig. 7.4b) for parallel 7.5a and perpendicular 7.5b polarization of the input laser is presented. The plasmon current is detected only when the laser is polarized parallel to the nanowire axis indicative of nanowire plasmon excitation. A polar graph of the magnitude of plasmon current as a function of excitation polarization is presented in Fig. 7.5c. When the excitation polarization is parallel $\left(\left|I_{\text {par }}\right|\right)$ and perpendicular $\left(\left|I_{\text {perp }}\right|\right)$ to the wire, the current contrast $\left(\left|I_{\text {par }}\right|-\left|I_{\text {perp }}\right|\right) /\left(\left|I_{\text {par }}\right|+\left|I_{\text {perp }}\right|\right)$ is calculated to be $66 \%$. This contrast exhibits the conversion efficiency of light into optical plasmon modes. The wires used in our study support two lower-order modes [194]. The fundamental mode, excited 
by light polarized parallel to the wire, has a higher in-coupling efficiency and $1 / e$ propagation length. When light is polarized perpendicular to the wire, plasmon throughput in the wire decreases, reducing the magnitude of the detected plasmon current. Fig. 7.5d shows the normalized polarization dependence of the photocurrent generated when the laser is focused at the $\mathrm{MoS}_{2} /$ wire interface for device 1 (blue curve, circles) and the $\mathrm{MoS}_{2}$ alone from device 2 (green curve, squares). Although the magnitude of the photocurrent is greater than the plasmon current, the polarization contrast is smaller and is calculated to be $29 \%$, with the maximum current for parallel polarization. This contrast is greater than from the $\mathrm{MoS}_{2}$ alone (12\% for Device 1 and $1 \%$ for Device 2$)$. This indicates a polarizationdependent mechanism for the photocurrent response of the $\mathrm{MoS}_{2} /$ wire interface. One possibility is that the wire acts like an antenna (Similar effect as presented in section 7.1) to enhance the photo-excitation of electron-hole pairs in the $\mathrm{MoS}_{2}$ [197] when light is polarized parallel to the nanowire axis.

\subsubsection{Figure of merits of the detector}

Two of the studied figure of merits for our near-field detector is the responsivity and plasmon-to-charge conversion efficiency. Responsivity is defined as the ratio between photocurrent generated in a detector and incident power. The responsivity is shown in Fig. 7.6a for both detection of far-field photons incident on the uncovered nanowire end ( $R_{p h}$, left y axis) and for detection of near-field plasmons $\left(R_{\text {plas }}\right.$, right y axis) and are calculated using the following equations [179]:

$$
\begin{gathered}
R_{p h}=\frac{I_{p h}}{P_{\text {in }}}, \\
R_{\text {plas }}=\frac{I_{\text {plas }}}{P_{\text {in }} * \alpha_{\text {in }} * e^{\frac{-L}{L_{0}}}}
\end{gathered}
$$



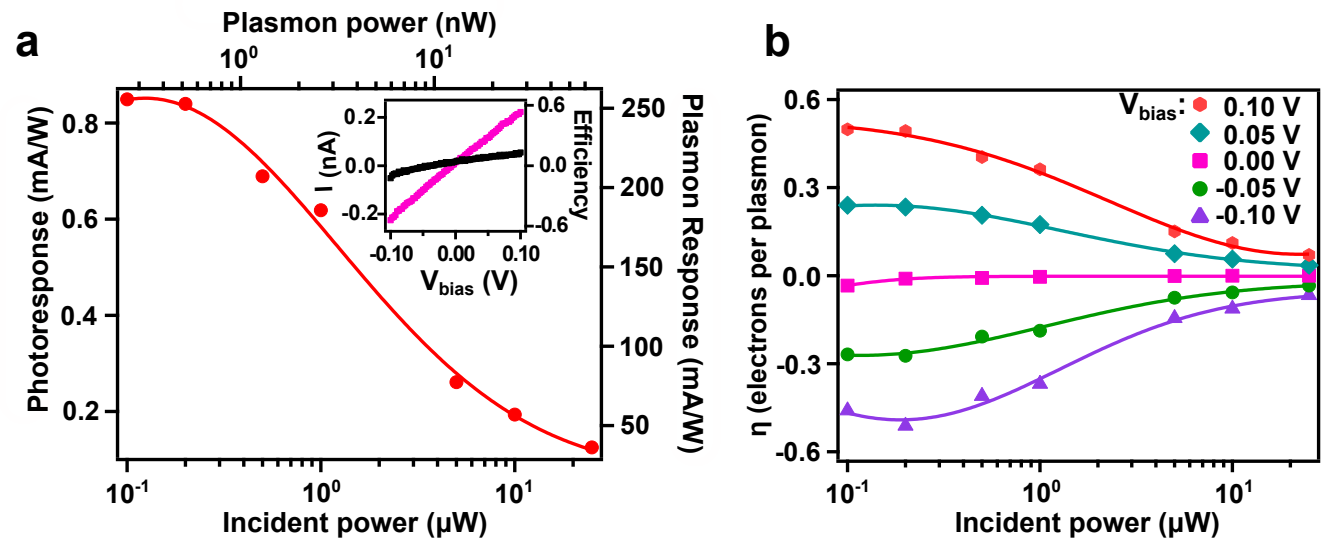

Figure 7.6: Responsivity and efficiency: a. Responsivity of plasmon current detection as a function of incident laser power for $\mathrm{V}_{\text {bias }}=0.1 \mathrm{~V}$. Inset: Plasmon current at excitation power of $200 \mathrm{nW}$ (pink) and dark current (black) as a function of source-drain bias voltage. The plasmon current curve has a larger slope than the dark current. b. Plasmon-to-charge conversion efficiency as a function laser power and bias voltage. For all data, $\lambda=635 \mathrm{~nm}$. Curves in the figure panels are guides to the eye. Figure adapted from [184] with permission.

where $\mathrm{L}$ is the length of the wire, $\alpha_{i n}$ is the photon-to-plasmon conversion efficiency, The bottom abscissa is presented in terms of incident power $P_{i n}$ and the top abscissa is the calculated plasmon power reaching the $\mathrm{MoS}_{2}$ detector calculated form the plasmon to photon conversion efficiency [179]. Our detector has a plasmon responsivity of $255 \mathrm{~mA} / \mathrm{W}$ at an optical power of $100 \mathrm{nW}$. The inset to Fig. 7.6a displays the plasmon current as a function of bias voltage and the detector dark current. The increase in the plasmon current when the bias is changed from zero is mediated by a plasmon-induced gating effect, similar to photogating in $\mathrm{MoS}_{2}$ phototransistors [202, 201]. 
a

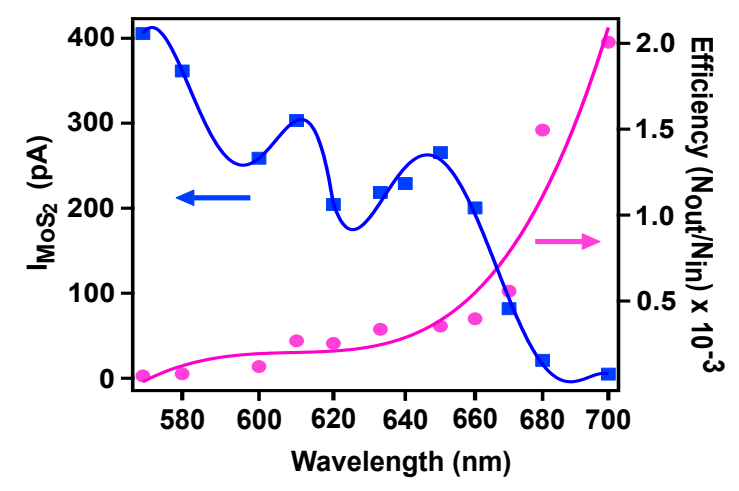

b

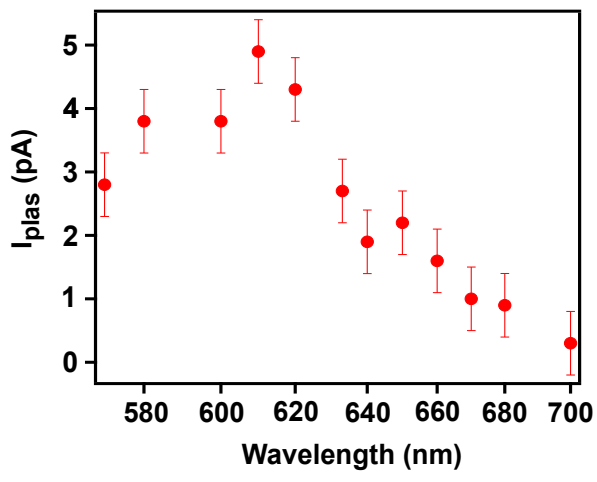

Figure 7.7: Wavelength dependent photocurrent: a. Normalized coupling efficiency of a bare wire (pink circles) and photocurrent of the $\mathrm{MoS}_{2}$ (blue squares) as a function of excitation wavelength. Curves in the figure panel are guides to the eye. b. Plasmon current as a function of excitation wavelength. For both panels, $\mathrm{V}_{\text {bias }}=5 \mathrm{mV}$, power $=2 \mu \mathrm{W}$. Figure adapted from [184] with permission.

The plasmon-to-charge conversion efficiency $(\eta)$ can be evaluated by [179]:

$$
\eta=\left(\frac{I_{\text {plas }}}{e}\right)\left(\frac{h c / \lambda_{e x}}{e^{\frac{-x}{L_{0}}} \alpha_{i n} P}\right),
$$

where $I_{\text {plas }}$ is the plasmon current, $e$ is the electron charge, $h c / \lambda_{e x}$ is the photon excitation energy, $x$ is the length of the nanowire from the uncovered end to the $\mathrm{MoS}_{2}, L_{0}$ is the estimated $1 / e$ propagation length for the wire, $\alpha_{i n}$ is the photonto-plasmon in-coupling efficiency, and $P$ is the optical power. We estimate the values for $\alpha_{i n}=0.13$ and $L_{0}=2.08 \mu \mathrm{m}$. For the data in the inset to Figure 7.6a, we determine $\eta$ to have a maximum magnitude of 0.5 at $\mathrm{V}_{\text {bias }}=-0.1 \mathrm{~V}$. Figure 7.6b demonstrates the variation of $\eta$ as a function of both $\mathrm{V}_{\text {bias }}$ and power. Consistent with previous observations [201], the detector efficiency increases for higher bias and lower optical power.

Next, we measured the spectral responsivity of our near-field plasmon detectors. Our hybrid devices contain two constituent elements with distinct spectral 
responses: the flake absorption and the wire transmission. Figure 7.7a displays the efficiency of photon throughput for a silver nanowire (pink curve, circles) and the measured photocurrent (blue curve, squares) when the laser is focused on the $\mathrm{MoS}_{2}$ (as measured for a second device) as a function of wavelength. To measure the bare wire throughput, a wire of similar length to that used in Device 2 was characterized. The 1/e propagation length of $\mathrm{Ag}$ nanowires, and the efficiency of photon re-emission after plasmon propagation, increases as the wavelength of light increases. In contrast, the photoresponsivity of the $\mathrm{MoS}_{2}$ decreases as the wavelength increases. The two peaks in responsivity around $610 \mathrm{~nm}$ and $650 \mathrm{~nm}$ are close to the absorption peaks in $\mathrm{MoS}_{2}$ that correspond to the direct $\mathrm{B}$ and A excitonic transitions [38]. Photoresponsivity becomes low beyond $670 \mathrm{~nm}$ due to the direct band gap of $\mathrm{MoS}_{2}$. Figure 7.7b displays the plasmon current as a function of wavelength for Device 2. As the wavelength is increased, plasmon propagation becomes more efficient, resulting in more electron-hole pairs to drive a current. The plasmon current peaks at $610 \mathrm{~nm}$, beyond which the detector becomes more characteristic of $\mathrm{MoS}_{2}$ detectors. For longer wavelengths, the $\mathrm{MoS}_{2}$ is less absorptive although more plasmons propagate through the nanowire.

\subsubsection{Summary}

In this section, we have demonstrated near-field electrical detection of Ag nanowire plasmons by monolayer $\mathrm{MoS}_{2}$. Applying a gate voltage to the detector channel to amplify the plasmon-generated current and modulate the plasmon-to-charge conversion efficiency is a natural next step. Exploration into leveraging atomicallythin semiconductors for on-chip light generation should also be possible. A final exciting direction will be to interface van der Waals heterostructures [44] with nanoplasmonic elements to realize fully-functional integrated nanophotonics. 


\subsection{Exciton polariton in 2D TMDC}

In this section, we study the strong coupling of cavity photons with the neutral and charged excitonic resonances of monolayer molybdenum diselenide $\left(\mathrm{MoSe}_{2}\right)$ embedded in a monolithic optical microcavity based on distributed Bragg's reflectors. The many body interactions lead to multiple polaritonic resonances and inverted band dispersion for the lowest trion derived branch [204]. These results suggests an interesting route for studying many -body physics and interesting devices based on cavity polaritonics [205, 49, 206, 207].

\subsubsection{Introduction}

Embedding an optically active semiconductor in a microcavity results in a myriad of fascinating effects related to resonant coupling of the photons trapped inside the cavity with the elementary optical excitation in the semiconductor such as excitons. In 1992, it had been experimentally demonstrated by Weisbuch et al [208], that, in the strong coupling regime the interaction between the exciton state in semiconductor and confined light mode in the microcavity can lead to the formation of completely two new eigenstates which have different properties than the bare exciton or the photon states. These collective excitation comprising part-light part-matter quasiparticles are known as exciton polaritons [209]. Since this pioneering work, a number of associated excitonic phenomenon such as Bose-Einstein condensation, superfluidity, polariton amplification, polariton lasing, polariton spin-switching, spin vortices, etc, have been discovered in traditional solid-state systems in microcavities. However, microcavity exciton polaritons in 2D materials have been only recently demonstrated [210]. Moreover, in all these work, the focus has been mostly on neutral excitons with only few efforts with 
other exciton complexes $[211,212]$. In this section, we will demonstrate the interaction of exciton, trion and cavity photon in an optical microcavity.

\subsubsection{Device architecture}

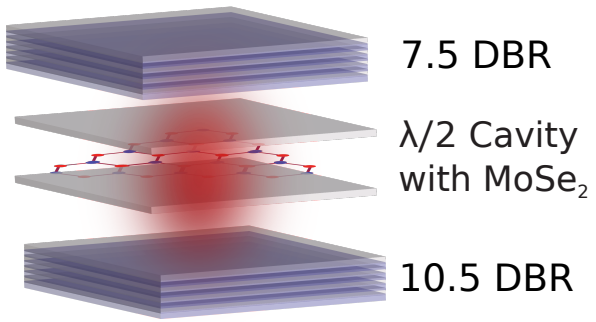

Figure 7.8: Schematic of the DBR based cavity hosting the monolayer $\mathrm{MoSe}_{2}$ flake at the center of the cavity.

As shown in Fig. 7.8, the microcavity consists of a monolayer $\mathrm{MoSe}_{2}$, sandwiched between two $\mathrm{SiO}_{2}$ layers to form the cavity which is placed between $\mathrm{SiO}_{2} / \mathrm{Ta}_{2} \mathrm{O}_{5}$ distributed Bragg reflector (DBR) mirrors grown via physical vapor deposition in a Kurt Lesker PVD-75 system. First the bottom DBR (10.5 pairs) and the bottom cavity $\left(\lambda_{0} / 4 \mathrm{~nm}\right)$ is grown on a silicon substrate. Following that, the $\mathrm{MoSe}_{2}$ flake was exfoliated using scotch tape (Semicorp brand) and transferred to the half cavity using the PDMS dry transfer technique [48]. Then the top cavity $\left(\lambda_{0} / 4 \mathrm{~nm}\right)$ and top mirror (7.5 pairs) are evaporated to complete the microcavity. The evaporation speed was maintained $1 \stackrel{\circ}{A} / s$.

\subsubsection{Optical characterization}

To determine the optical quality of the exfoliated flakes typically used in the experiments, reflectivity and photoluminescence measurements were carried out 

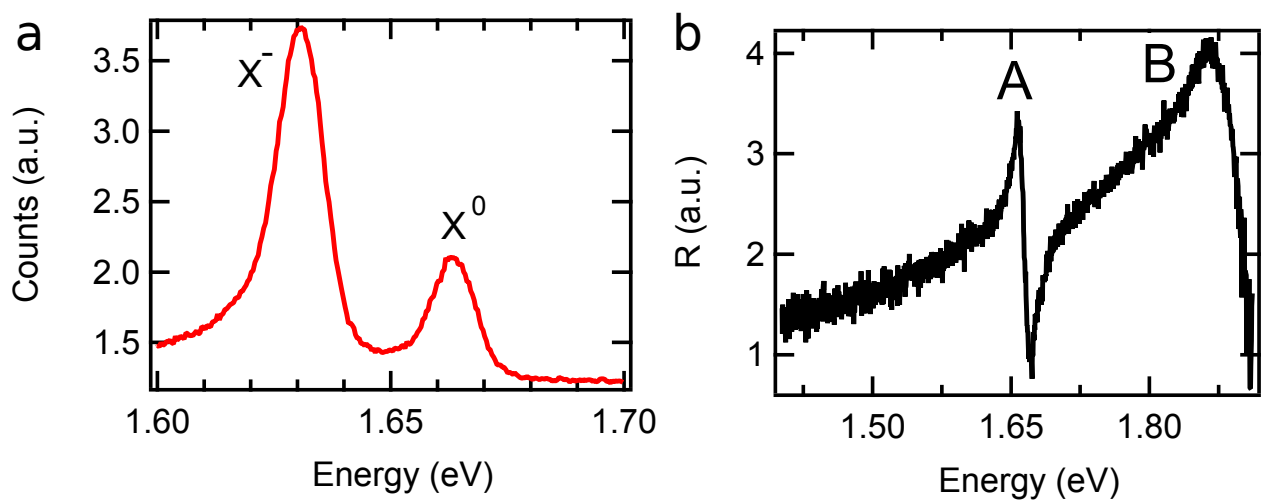

Figure 7.9: Optical characterization of bare $\mathrm{MoSe}_{2}$ : a. PL and b. differential reflectance spectrum of a $\mathrm{MoSe}_{2}$ flake at $4 \mathrm{~K}$.

on a monolayer $\mathrm{MoSe}_{2}$ flake exfoliated on $\mathrm{SiO}_{2}$ substrate. The photoluminescence spectra from a monolayer $\mathrm{MoSe}_{2}$ flake is shown in Fig 7.9a. The spectra shows two dominant resonances - the exciton at $\sim 1.658 \mathrm{eV}$ and trion at $\sim 1.625 \mathrm{eV}$. The exciton (trion) linewidth is $7 \mathrm{meV}(9 \mathrm{meV})$. The reflectance spectra from the $\mathrm{MoSe}_{2}$ reveals the two prominent absorption peaks identified as the $\mathrm{A}$ and the $\mathrm{B}$ exciton respectively. Note that the trion resonance is absent in the reflectance spectra. This observation is similar as earlier experiments [213] possibly due to lower density of trions without external doping.

Reflectance spectrum of the bare cavity (region where there is no $\mathrm{MoSe}_{2}$ layer) shows that the dip at the cavity resonance is around $1.658 \mathrm{eV}$ (Fig 7.10a). The linewidth of the resonance is around $3 \mathrm{meV}$ and a quality factor of 550 . The PL spectrum of the loaded cavity region shows three distinct peaks shifted from the bare exciton and bare cavity indicating the formation of a new eigenstate. The spectra in Fig $7.10 \mathrm{~b}$ can be fit to 3 Lorentzian peaks which originate from the upper, middle and lower polariton branch respectively. 
a

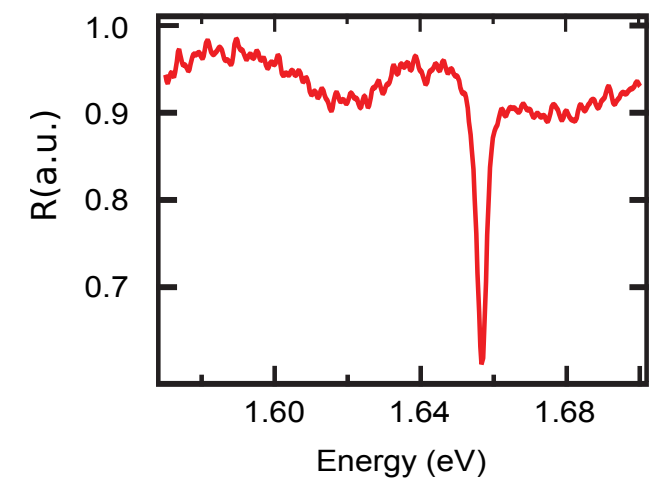

b Loaded cavity

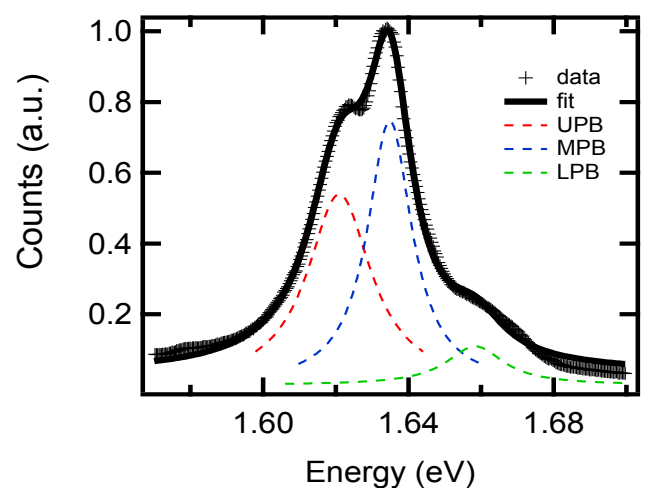

Figure 7.10: Bare cavity vs loaded cavity: a. Reflectance from empty cavity at $4 \mathrm{~K}$ b. PL spectrum of the part of the cavity loaded with the $\mathrm{MoSe}_{2}$ flake at $4 \mathrm{~K}$.

\subsubsection{Imaging the dispersion}

\section{Optical setup}

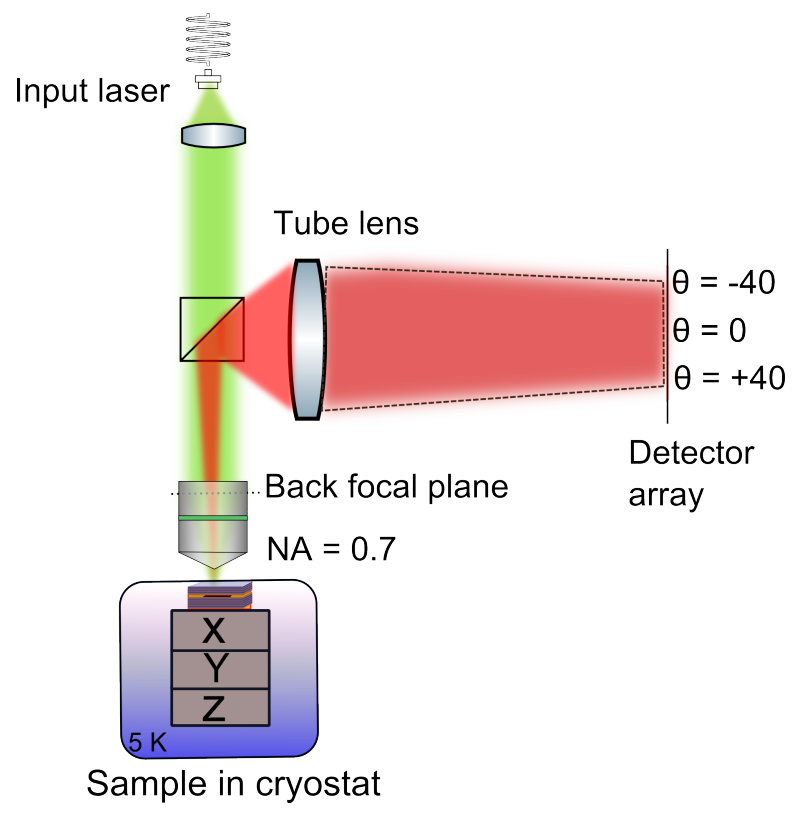

Figure 7.11: Schematic of the setup used to image the dispersion.

To confirm the formation of polariton states, angle resolved measurements 
were performed. The schematic of our experimental setup is shown in Fig. 7.11. A horizontal dotted line is used to represent the position of the back focal plane or the Fourier plane in the $60 \mathrm{X}$ objective lens (Nikon) which has the numerical aperture of 0.7 . Ideally, this objective lens can allow us to collect the PL roughly within an angular range of $\pm 44^{0}$ without vignetting. Therefore, each point in the Fourier plane corresponds to a particular angle of PL emission. We use a tube lens to image the back focal plane of the objective lens on to the wide aperture slit of an Acton (Princeton Instrument) spectrometer with $750 \mathrm{~mm}$ focal length. We use a CCD camera with an array of 1340 x 1300 pixels (with $20 \mu \mathrm{m}$ pixel size) to perform the imaging as well as spectroscopy. If we consider a vertical line along the slit aperture of the spectrometer which is directly imaged on to the CCD sensor, each pixel on the vertical axis of the CCD contains the angular information at the same time the emission is dispersed using a grating of 150 lines/mm, therefore each pixel in the horizontal direction contains the spectral information.

\section{Calibration}

Before we performed our experiment, we calibrated our setup using a bare microcavity sample. A typical angle resolved reflectance spectrum that we get from the CCD sensor is shown in Fig. 7.12a above, where we have transposed the raw data in order to get the vertical pixels as energy and the horizontal axis proportional to the angle of reflection. To calibrate, we use the bare cavity photon dispersion given by: $\lambda(\theta)=\lambda_{0} \cos (\theta)$, where, $\lambda$ is the wavelength, $\theta$ is the angle of incidence/reflection and $\lambda_{0}$ is the resonant wavelength of the cavity at normal incidence. After calibration, we find that the maximum angular range that we can investigate in our setup is around $\pm 24^{0}$, this reduction in the allowed angular range for a $0.7 \mathrm{NA}$ objective $\left( \pm 44^{0}\right)$ is due to vignetting by the back aperture of 

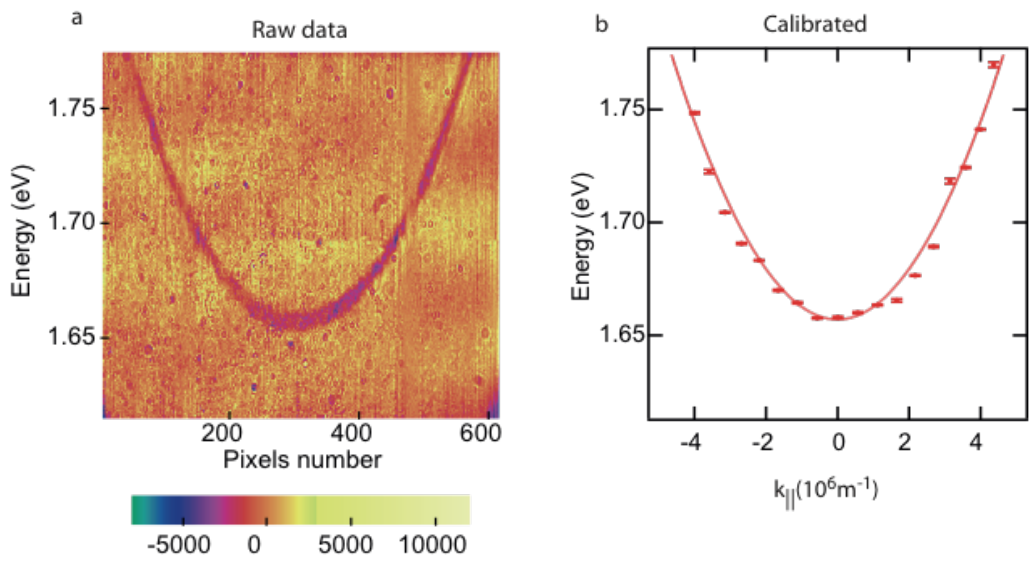

Figure 7.12: Setup calibration: a. Bare cavity dispersion as captured by the spectrometer CCD as a function of pixel numbers on the camera b. Data points obtained from fitting the reflectance line plot at different pixel points to get an angular calibration for the abscissa.

the objective lens. We find that the total number of pixels that spans over the angular range of $\pm 24^{0}$ is around 640 , which gives an angular resolution $\delta \theta$ for our setup around $\sim 0.1^{0}$. The error associated with the spectrometer for energy measurement is only limited by the number of lines/mm $(150 \mathrm{l} / \mathrm{mm})$ of the grating used. The error bar in the energy scale is around $0.4 \mathrm{meV}$.

\subsubsection{The cavity coupled $\mathrm{MoSe}_{2}$}

The raw angle-resolved spectrum is shown in Fig. 7.13a, where the color represents the differentiated intensity of $\mathrm{PL}$ in $\log$ scale. Three distinct branches corresponding to upper (UPB), middle (MPB), and lower (LPB) polariton are observed. The upper and the middle polariton branch has a positive dispersion, however, the lower polariton branch reveals a negative dispersion in the $\mathrm{E}$ vs $\mathrm{k}$ plot. This is highlighted by the line-cuts taken from the raw data presented in 

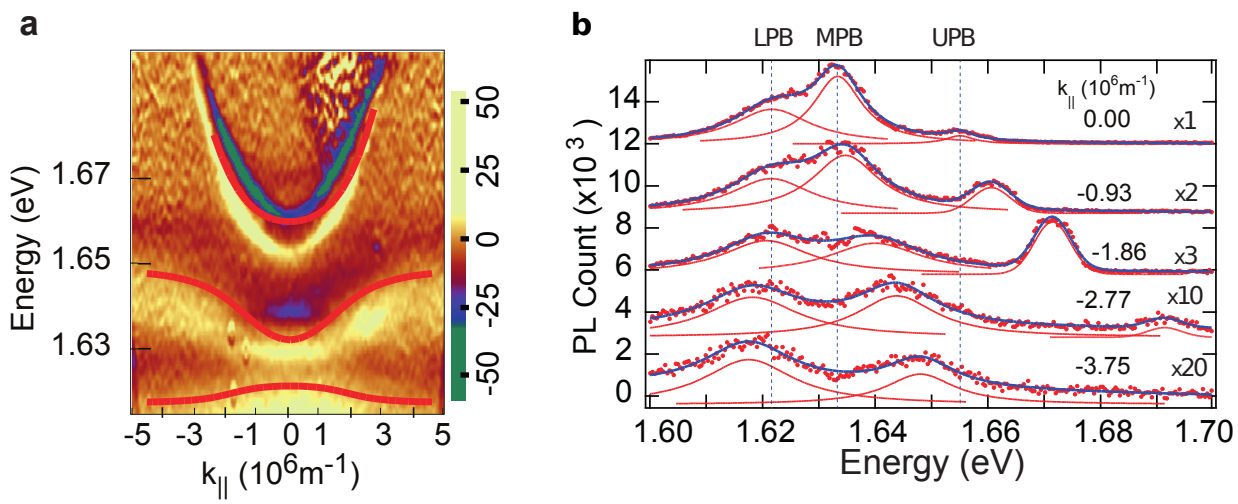

Figure 7.13: Angle resolved PL: a. A single-shot angle-resolved photoluminescence (PL) measurement of our device obtained at $6 \mathrm{~K}$; the logarithm of the raw data is differentiated to enhance the contrast b. PL spectrum as taken from the line cuts at five different $\mathrm{k}_{||}$exhibiting negative dispersion for the LPB. Vertical blue dashed lines indicate peak position at $k_{\|}=0$. Figure adapted from [204] with permission.

Fig. 7.13b. While the peaks corresponding to UPB and MPB blue shifts from zero momentum value $\left(\mathrm{k}_{\|}=0\right)$, the LPB redshifts owing to the negative dispersion. Each of this PL spectrum was fitted with Lorentzian line shapes to obtain the peak positions corresponding to the lower, middle and upper polaritons. The peak energies from the individual polariton branch are plotted in Fig. 7.14a in the next section 7.3.6.

\subsubsection{Deviation from positive dispersion}

After extracting the energies from the dispersion plot, the effect of level repulsion between the photon and the neutral exciton branch is clear (Fig 7.14a). Here, the upper and middle polariton has a positive dispersion. However, the lower trion branch deviates from this trend and gives an inverted dispersion. To arrive at an understanding of the interaction between the excitons with the cavity photons, 

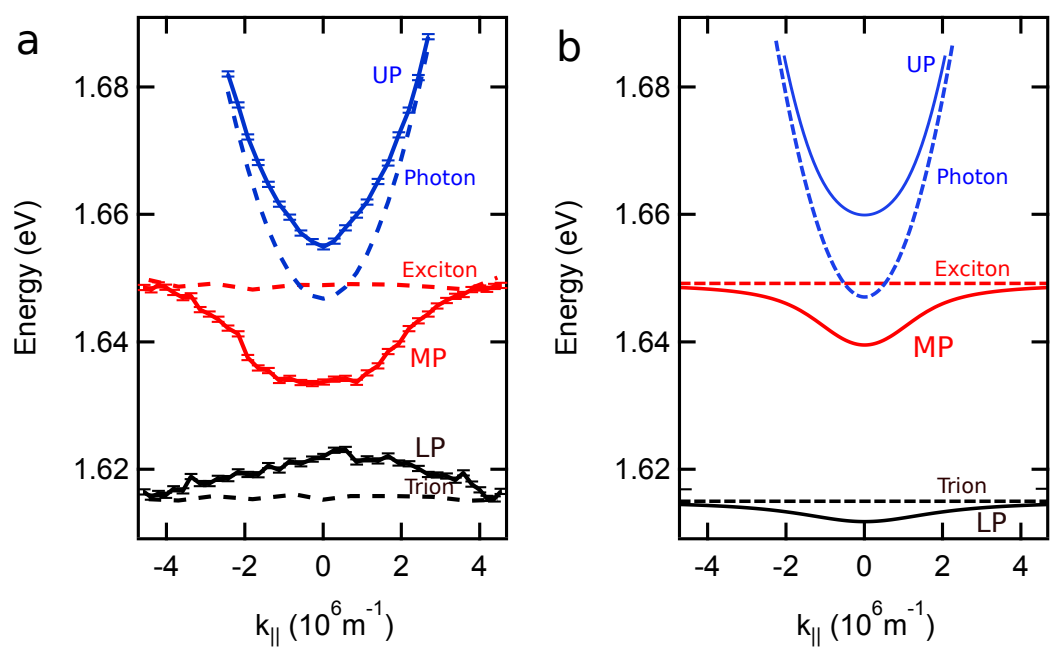

Figure 7.14: Experiment vs coupled three oscillator model: a. Energy points extracted from the line-cuts taken from raw data and b. coupled three oscillator model 7.4. The dashed lines are the dispersion of the bare $\mathrm{MoSe}_{2}$ exciton, trion and cavity photon obtained at $6 \mathrm{~K}$ and the solid lines are data obtained from modeling 3 coupled oscillator or directly from the polariton branches in the experiment.

we compare our experiment to a coupled oscillator model. Below is the standard coupled oscillator model extended to three particles that is usually solved to obtain the dispersion of the polariton modes resulting from normal mode coupling of two different exciton species with cavity photon [214]:

$$
\left[\begin{array}{ccc}
E_{e x} & 0 & \frac{v 1}{2} \\
0 & E_{t r} & \frac{v 2}{2} \\
\frac{v 1}{2} & \frac{v 2}{2} & E_{p h}
\end{array}\right]\left[\begin{array}{l}
\alpha \\
\beta \\
\gamma
\end{array}\right]=E\left[\begin{array}{l}
\alpha \\
\beta \\
\gamma
\end{array}\right]
$$

The three coefficients $\alpha, \beta$ and $\gamma$ quantify the admixture of monolayer-excitons and photon and, $E_{e x}, E_{t r}$ and $E_{p h}$ are the uncoupled energies of the exciton, trion and cavity photon. The expression of the bare energies are obtained from ref [215]. v1 (v2) is the exciton-photon (trion-photon) interaction strength. E represents 
the eigenvalues corresponding to the energies of the polariton modes.

Using this model, we obtain three polariton branches with positive dispersion. The upper and the middle polariton branch in our experimental data match the trend of this coupled oscillator model. However, the lower, trion branch deviates from this model to give an anomalous inverted dispersion. This inverted dispersion has been attributed to electron-mediated level attraction between the excitonpolariton branch and the trion branch that overwhelms the weak trion cavity repulsion, leading to level attraction. The origin of such an interaction could be due to delocalization of an electron between a neutral exciton and the charged exciton (trion) [204].

\subsubsection{Summary}

In conclusion, we have observed clear evidence for the formation of exciton polaritons in a dielectric cavity device featuring an integrated single atomic layer of the TMDC $\mathrm{MoSe}_{2}$. We mapped out the distinct polariton dispersion relation in single shot angle-resolved PL measurements, and resolved all three polariton branches including the characteristic energy minimum and the flattening towards the exciton band. The dispersion of the trion derived polariton branch presents an anomalous dispersion from our measurements. Polariton devices have not only become a perfect workbench for studying fundamental physics but also a prominent technology for modern quantum optoelectronics [206]. 


\section{Chapter 8}

\section{Conclusion and perspective}

The purpose of this chapter will be to summarize the key results for each topic in this dissertation. Following that, a concluding section with a brief perspective on possible future studies that can be realized and explored with TMDCs will be presented.

\subsection{Overall summary}

The discovery of the localized exciton states in TMDCs and several ways of controlling their optical properties by leveraging in electrically tunable van der Waals heterostructures are chronicled in this thesis. Availing the imperfections in the 2D lattice, one can convert the 1D confinement potential for the excitons in a monolayer flake to a 3D confined one for TMDCs like $\mathrm{WSe}_{2}$ and $\mathrm{MoSe}_{2}$ at low temperatures. In certain aspects, this is similar to semiconductor heterojunctions where interface imperfections are created by lattice mismatch for self-assembled quantum dots. Several external parameters like, electric field, magnetic field and even strain fields have been used to demonstrate their tunability. Low temperature micro-photoluminescence measurements are used to characterize most of their 
photophysical properties as described in chapters $3-6$. Antibunching is confirmed by performing second-order intensity autocorrelation measurements. Polarizationresolved and magneto-optical photoluminescence studies reveal a large zero-field splitting up to $\sim 0.8 \mathrm{meV}$ and an exciton g-factor up to $\sim 10$. van der Waals heterostructure have been built with these emitters to demonstrate electrical manipulation of their optical transition properties like energy, line width, intensity and even the fine structure splitting. Such tunability is required for any practical utilization. For example, it would always be desirable to tune the resonance of the emitters if integrated with an optical cavity or with respect to each other for applications in quantum information processing. This can be achieved via the quantum confined Stark effect which is demonstrated by these emitters embedded in van der Waals heterostructures. A maximum peak energy modulation of 21 $\mathrm{meV}$ is achieved in these localized emitters by electric field. Furthermore, electrostatic control of charge carriers in the localized emitters have also been supported by charge tunable van der Waals devices. This made it possible to confine a trion to these localized states. Single spins localized in semiconductors have been considered as qubits in quantum computing and quantum spintronics. Thus, trions in TMDCs can enable optically addressable spin-valley qubits by single localized electrons for valleytronic applications.

In addition, few optoelectronic and nanophotonic devices with 2D materials are also studied which would serve as future building block for on-chip photonic circuits with 2D materials. Integrated plasmonic photodetectors have been studied with monolayer graphene and $\mathrm{MoS}_{2}$ with metallic nano-antenna and nanowire respectively. Such devices have demonstrated the ease with which 2D materials can be coupled to plasmonic structures for photodetection with high responsivities. Finally, microcavity polaritons have been studied by coupling light with 
neutral and charged excitons of monolayer $\mathrm{MoSe}_{2}$. The strong coupling between the exciton, trion and photon in the cavity results in upper and middle polariton branches with positive dispersion and a lower, trion-derived polariton branch with an anomalous dispersion.

\subsection{Perspective}

As indicated by the breadth of interesting properties and applications highlighted in this thesis, there is a great deal of promise for nanophotonics based on 2D materials. All these studies are reason enough to justify the enthusiasm of the 2D materials scientific community. More recently, the discovery of single photon emitters in 2D materials, as presented in this thesis, has also stirred the interest of the quantum optics community in 2D materials based research.

Firstly, single photon emission from 2D material could engender rapid developments in easy and low-cost single photon sources for fundamental quantum optics studies. If nothing else, exfoliating a flake on a substrate sure seems to be one of the quickest recipe for making a single photon emitting source. The two-dimensional host of the emitters would offer additional advantages over other solid-state emitters. Photons from emitters localized in a 3D matrix usually have to travel through the 3D host resulting in losses before they can be collected for further applications. However, for the case of the 2D host, the photons can be extracted with a greater extraction efficiency. Moreover, their 2D nature lets one position the localized emitters as close as possible to any test surface enhancing the efficiency of sensing based applications. Also, strain induced generation of these emitters is made possible by standard lithographic $[85,86,91]$ or even mechanical transfer techniques [216]. This has also demonstrated that the origin 
of these emitters is extrinsic to the TMDC flake. This facilitates deterministic positioning and integration with photonic cavities for cavity QED experiments.

However, some of the most important breakthrough in science and technology have resulted from a deeper understanding of the properties of materials. Therefore, a complete knowledge about the origin of the quantum confined emitters in 2D materials is strongly necessary. While there are few experiments and theory that predicts the origin of these emitters, a more conclusive experimental verification backed up by results from theoretical study is required for unmasking the true identity of these emitters. The remaining challenges are room temperature operations and wafer-sized scalability of the production of these localized states. While the former can be made possible by utilizing defects like Nitrogen-vacancy centers present in insulating h-BN monolayers that are stable even at room temperature or engineering other defects in 2D materials with deep confinements, the latter can be only achieved with well controlled wafer-scale growth techniques of 2D materials.

There are also many exciting opportunities yet to be explored with 2D materials beyond the quantum emitters. One of them is in regard to the development of new 2D materials and proficient growth techniques to produce wafer scale samples with high purity. Although 2D materials themselves can be found in a wide variety of properties, their true potential could be realized by tunable van der Waals heterostructure based devices. Integrated nanophotonics like microcavity polaritons for low threshold lasers are also desirable. Although, they compete directly with GaAs based conventional lasers [217], their potential application in quantum information processing with valley and spin properties are in particular attractive. Earlier optically pumped vertical cavity surface emitting laser have demonstrated the operation of a low threshold spin laser with InGaAs quantum wells [218]. It 
is predicted that this concept can be generalized to spin-valley lasers with TMDC coupled with microcavities while utilizing their valley optical selection rules [219].

Although the discussions for applications of 2D materials is still long on speculation but that is only because this research is still in its infancy. It requires many more years of work similar to most other successful fields after which it will not be far from practical utilization. 


\section{Bibliography}

[1] S. Iijima, Nature 354, 56 (1991).

[2] H. W. Kroto, J. R. Heath, S. C. O'Brien, R. F. Curl, and R. E. Smalley, Nature 318, 162 (1985).

[3] B. C. Brodie, Philosophical Transactions of the Royal Society of London 149, 249 (1859).

[4] K. S. Novoselov, A. K. Geim, S. V. Morozov, D. Jiang, Y. Zhang, S. V. Dubonos, I. V. Grigorieva, and A. A. Firsov, Science 306, 666 (2004).

[5] S. Ulstrup, J. C. Johannsen, F. Cilento, J. A. Miwa, A. Crepaldi, M. Zacchigna, C. Cacho, R. Chapman, E. Springate, S. Mammadov, F. Fromm, C. Raidel, T. Seyller, F. Parmigiani, M. Grioni, P. D. C. King, and P. Hofmann, Physical Review Letters 112, 257401 (2014).

[6] G. R. Bhimanapati, Z. Lin, V. Meunier, Y. Jung, J. Cha, S. Das, D. Xiao, Y. Son, M. S. Strano, V. R. Cooper, L. Liang, S. G. Louie, E. Ringe, W. Zhou, S. S. Kim, R. R. Naik, B. G. Sumpter, H. Terrones, F. Xia, Y. Wang, J. Zhu, D. Akinwande, N. Alem, J. A. Schuller, R. E. Schaak, M. Terrones, and J. A. Robinson, ACS Nano 9, 11509 (2015).

[7] G. Giovannetti, P. A. Khomyakov, G. Brocks, P. J. Kelly, and J. van den Brink, Physical Review B 76, 073103 (2007). 
[8] M. Chhowalla, H. S. Shin, G. Eda, L.-J. Li, K. P. Loh, and H. Zhang, Nature Chemistry 5, 263 (2013).

[9] S. Stankovich, D. A. Dikin, G. H. B. Dommett, K. M. Kohlhaas, E. J. Zimney, E. A. Stach, R. D. Piner, S. T. Nguyen, and R. S. Ruoff, Nature 442, 282 (2006).

[10] A. O’Hare, F. V. Kusmartsev, and K. I. Kugel, Nano Letters 12, 1045 (2012).

[11] V. Nicolosi, M. Chhowalla, M. G. Kanatzidis, M. S. Strano, and J. N. Coleman, Science 340, 1226419 (2013).

[12] A. K. Geim and I. V. Grigorieva, Nature 499, 419 (2013).

[13] T. Tashiro, A. Ishida, M. Hori, M. Igisu, M. Koike, P. Méjean, N. Takahata, Y. Sano, and T. Komiya, Nature 549, 516 (2017).

[14] M. Pozzo, D. Alfè, P. Lacovig, P. Hofmann, S. Lizzit, and A. Baraldi, Physical Review Letters 106, 135501 (2011).

[15] M. C. Lemme, Solid State Phenomena 156-158, 499 (2010).

[16] D. S. Hecht, L. Hu, and G. Irvin, Advanced Materials 23, 1482 (2011).

[17] K. S. Novoselov and A. H. C. Neto, Physica Scripta 2012, 014006 (2012).

[18] P. Blake, E. W. Hill, A. H. Castro Neto, K. S. Novoselov, D. Jiang, R. Yang, T. J. Booth, and A. K. Geim, Applied Physics Letters 91, 063124 (2007).

[19] K. Kneipp, H. Kneipp, I. Itzkan, R. R. Dasari, and M. S. Feld, Chemical Reviews 99, 2957 (1999). 
[20] H. Li, Q. Zhang, C. C. R. Yap, B. K. Tay, T. H. T. Edwin, A. Olivier, and D. Baillargeat, Adv. Funct. Mater. 22, 1385 (2012).

[21] C. V. Raman and K. S. Krishnan, Nature 121, 501 (1928).

[22] R. Saito, M. Hofmann, G. Dresselhaus, A. Jorio, and M. S. Dresselhaus, Advances in Physics 60, 413 (2011).

[23] A. Das, B. Chakraborty, and A. K. Sood, Bulletin of Materials Science 31, 579 (2008).

[24] J. Xue, J. Sanchez-Yamagishi, D. Bulmash, P. Jacquod, A. Deshpande, K. Watanabe, T. Taniguchi, P. Jarillo-Herrero, and B. J. LeRoy, Nature Materials 10, 282 (2011).

[25] K. Watanabe, T. Taniguchi, and H. Kanda, Nature Materials 3, 404 (2004).

[26] C. R. Dean, A. F. Young, I. Meric, C. Lee, L. Wang, S. Sorgenfrei, K. Watanabe, T. Taniguchi, P. Kim, K. L. Shepard, and J. Hone, Nature Nanotechnology 5, 722 (2010).

[27] J. Wang, F. Ma, and M. Sun, RSC Advances 7, 16801 (2017).

[28] L. Song, L. Ci, H. Lu, P. B. Sorokin, C. Jin, J. Ni, A. G. Kvashnin, D. G. Kvashnin, J. Lou, B. I. Yakobson, and P. M. Ajayan, Nano Letters 10, 3209 (2010).

[29] Y. Cao, A. Mishchenko, G. L. Yu, E. Khestanova, A. P. Rooney, E. Prestat, A. V. Kretinin, P. Blake, M. B. Shalom, C. Woods, J. Chapman, G. Balakrishnan, I. V. Grigorieva, K. S. Novoselov, B. A. Piot, M. Potemski, K. Watanabe, T. Taniguchi, S. J. Haigh, A. K. Geim, and R. V. Gorbachev, Nano Letters 15, 4914 (2015). 
[30] R. Tenne, L. Margulis, M. Genut, and G. Hodes, Nature 360, 444 (1992).

[31] L. Margulis, G. Salitra, R. Tenne, and M. Talianker, Nature 365, 113 (1993).

[32] A. Rothschild, J. Sloan, and R. Tenne, Journal of the American Chemical Society 122, 5169 (2000).

[33] C. N. R. Rao and A. Govindaraj, Advanced Materials 21, 4208 (2009).

[34] K. S. Novoselov, D. Jiang, F. Schedin, T. J. Booth, V. V. Khotkevich, S. V. Morozov, and A. K. Geim, Proceedings of the National Academy of Sciences 102, 10451 (2005).

[35] Y. L. Huang, Y. Chen, W. Zhang, S. Y. Quek, C.-H. Chen, L.-J. Li, W.T. Hsu, W.-H. Chang, Y. J. Zheng, W. Chen, and A. T. S. Wee, Nature Communications 6, 6298 (2015).

[36] R. Suzuki, M. Sakano, Y. J. Zhang, R. Akashi, D. Morikawa, A. Harasawa, K. Yaji, K. Kuroda, K. Miyamoto, T. Okuda, K. Ishizaka, R. Arita, and Y. Iwasa, Nature Nanotechnology 9, 611 (2014).

[37] R. F. Frindt, Journal of Applied Physics 37, 1928 (1966).

[38] A. Splendiani, L. Sun, Y. Zhang, T. Li, J. Kim, C.-Y. Chim, G. Galli, and F. Wang, Nano Letters 10, 1271 (2010).

[39] M. Javaid, S. P. Russo, K. Kalantar-Zadeh, A. D. Greentree, and D. W. Drumm, arXiv:1711.03236 [cond-mat, physics:physics] (2017), arXiv: 1711.03236.

[40] M. Velický and P. S. Toth, Applied Materials Today 2D Materials in Electrochemistry, 8, 68 (2017). 
[41] H. C. P. Movva, A. Rai, S. Kang, K. Kim, B. Fallahazad, T. Taniguchi, K. Watanabe, E. Tutuc, and S. K. Banerjee, ACS Nano 9, 10402 (2015).

[42] M. Bernardi, M. Palummo, and J. C. Grossman, Nano Letters 13, 3664 (2013).

[43] P. Tonndorf, R. Schmidt, P. Böttger, X. Zhang, J. Börner, A. Liebig, M. Albrecht, C. Kloc, O. Gordan, D. R. T. Zahn, S. M. d. Vasconcellos, and R. Bratschitsch, Optics Express 21, 4908 (2013).

[44] A. K. Geim and I. V. Grigorieva, Nature 499, 419 (2013).

[45] F. Withers, O. Del Pozo-Zamudio, A. Mishchenko, A. Rooney, A. Gholinia, K. Watanabe, T. Taniguchi, S. Haigh, A. Geim, A. Tartakovskii, and K. Novoselov, Nature Materials 14, 301 (2015).

[46] C. Palacios-Berraquero, M. Barbone, D. M. Kara, X. Chen, I. Goykhman, D. Yoon, A. K. Ott, J. Beitner, K. Watanabe, T. Taniguchi, A. C. Ferrari, and M. Atatüre, Nature Communications 7, 12978 (2016).

[47] P. Rivera, J. R. Schaibley, A. M. Jones, J. S. Ross, S. Wu, G. Aivazian, P. Klement, K. Seyler, G. Clark, N. J. Ghimire, J. Yan, D. G. Mandrus, W. Yao, and X. Xu, Nature Communications 6, 6242 (2015).

[48] A. Castellanos-Gomez, M. Buscema, R. Molenaar, V. Singh, L. Janssen, H. S. J. v. d. Zant, and G. A. Steele, 2D Materials 1, 011002 (2014).

[49] H. Deng, H. Haug, and Y. Yamamoto, Reviews of Modern Physics 82, 1489 (2010).

[50] D. A. B. Miller, Optical Physics of Quantum Wells. 
[51] K. He, N. Kumar, L. Zhao, Z. Wang, K. F. Mak, H. Zhao, and J. Shan, arXiv:1406.3095 [cond-mat] (2014), arXiv: 1406.3095.

[52] K. Mak, K. He, C. Lee, G. H. Lee, J. Hone, T. F. Heinz, and J. Shan, Nature Materials 12, 207 (2013).

[53] Y. You, X.-X. Zhang, T. C. Berkelbach, M. S. Hybertsen, D. R. Reichman, and T. F. Heinz, Nature Physics 11, 477 (2015).

[54] D. Y. Qiu, F. H. da Jornada, and S. G. Louie, Physical Review Letters 111, 216805 (2013).

[55] B. Zhu, X. Chen, and X. Cui, Scientific Reports 5, 9218 (2015).

[56] C. Robert, D. Lagarde, F. Cadiz, G. Wang, B. Lassagne, T. Amand, A. Balocchi, P. Renucci, S. Tongay, B. Urbaszek, and X. Marie, Physical Review B 93, 205423 (2016).

[57] H. Zeng, J. Dai, W. Yao, D. Xiao, and X. Cui, Nature Nanotechnology 7, $490(2012)$.

[58] Q. H. Wang, K. Kalantar-Zadeh, A. Kis, J. N. Coleman, and M. S. Strano, Nature Nanotechnology 7, 699 (2012).

[59] K. Kośmider, J. W. González, and J. Fernández-Rossier, Physical Review B 88, 245436 (2013).

[60] M. Amani, D.-H. Lien, D. Kiriya, J. Xiao, A. Azcatl, J. Noh, S. R. Madhvapathy, R. Addou, S. Kc, M. Dubey, K. Cho, R. M. Wallace, S.-C. Lee, J.-H. He, J. W. Ager, X. Zhang, E. Yablonovitch, and A. Javey, Science 350, 1065 (2015). 
[61] P. Tonndorf, R. Schmidt, P. Böttger, X. Zhang, J. Börner, A. Liebig, M. Albrecht, C. Kloc, O. Gordan, D. R. T. Zahn, S. M. d. Vasconcellos, and R. Bratschitsch, Optics Express 21, 4908 (2013).

[62] T. Yan, X. Qiao, X. Liu, P. Tan, and X. Zhang, Applied Physics Letters 105, 101901 (2014).

[63] A. Chernikov, A. M. van der Zande, H. M. Hill, A. F. Rigosi, A. Velauthapillai, J. Hone, and T. F. Heinz, Physical Review Letters 115, 126802 (2015).

[64] D. Miller, D. Chemla, T. Damen, A. Gossard, W. Wiegmann, T. Wood, and C. Burrus, Phys. Rev. Lett. 53, 2173 (1984).

[65] J. R. Schaibley, H. Yu, G. Clark, P. Rivera, J. S. Ross, K. L. Seyler, W. Yao, and X. Xu, Nature Reviews Materials 1, 16055 (2016).

[66] D. Xiao, G.-B. Liu, W. Feng, X. Xu, and W. Yao, Phys. Rev. Lett. 108, $196802(2012)$.

[67] A. M. Jones, H. Yu, N. J. Ghimire, S. Wu, G. Aivazian, J. S. Ross, B. Zhao, J. Yan, D. G. Mandrus, D. Xiao, W. Yao, and X. Xu, Nature Nanotechnology 8, 634 (2013).

[68] H. Yu, X. Cui, X. Xu, and W. Yao, National Science Review 2, 57 (2015).

[69] A. Singh, K. Tran, M. Kolarczik, J. Seifert, Y. Wang, K. Hao, D. Pleskot, N. M. Gabor, S. Helmrich, N. Owschimikow, U. Woggon, and X. Li, Physical Review Letters 117, 257402 (2016).

[70] P. Rivera, K. L. Seyler, H. Yu, J. R. Schaibley, J. Yan, D. G. Mandrus, W. Yao, and X. Xu, Science 351, 688 (2016). 
[71] A. Srivastava, M. Sidler, A. V. Allain, D. S. Lembke, A. Kis, and A. Imamoğlu, Nature Physics 11, 141 (2015).

[72] Y. Li, J. Ludwig, T. Low, A. Chernikov, X. Cui, G. Arefe, Y. D. Kim, A. M. van der Zande, A. Rigosi, H. M. Hill, S. H. Kim, J. Hone, Z. Li, D. Smirnov, and T. F. Heinz, Physical Review Letters 113, 266804 (2014).

[73] D. MacNeill, C. Heikes, K. F. Mak, Z. Anderson, A. Kormányos, V. Zólyomi, J. Park, and D. C. Ralph, Physical Review Letters 114, 037401 (2015).

[74] C. Chakraborty, L. Kinnischtzke, K. M. Goodfellow, R. Beams, and A. N. Vamivakas, Nature Nanotechnology 10, 507 (2015).

[75] A. Srivastava, M. Sidler, A. V. Allain, D. S. Lembke, A. Kis, and A. Imamoğlu, Nature Nanotechnology 10, 491 (2015).

[76] Y.-M. He, G. Clark, J. R. Schaibley, Y. He, M.-C. Chen, Y.-J. Wei, X. Ding, Q. Zhang, W. Yao, X. Xu, C.-Y. Lu, and J.-W. Pan, Nature Nanotechnology 10, $497(2015)$.

[77] M. Koperski, K. Nogajewski, A. Arora, V. Cherkez, P. Mallet, J.-Y. Veuillen, J. Marcus, P. Kossacki, and M. Potemski, Nature Nanotechnology 10, 503 (2015).

[78] P. Tonndorf, R. Schmidt, R. Schneider, J. Kern, M. Buscema, G. A. Steele, A. Castellanos-Gomez, H. S. J. van der Zant, S. Michaelis de Vasconcellos, and R. Bratschitsch, Optica 2, 347 (2015).

[79] G. Wei, D. A. Czaplewski, E. J. Lenferink, T. K. Stanev, I. W. Jung, and N. P. Stern, arXiv:1510.09135 [cond-mat] (2015), arXiv: 1510.09135. 
[80] T. T. Tran, K. Bray, M. J. Ford, M. Toth, and I. Aharonovich, Nature Nanotechnology 11, 37 (2016).

[81] C. Chakraborty, K. M. Goodfellow, and A. Nick Vamivakas, Optical Materials Express 6, 2081 (2016).

[82] A. Branny, G. Wang, S. Kumar, C. Robert, B. Lassagne, X. Marie, B. D. Gerardot, and B. Urbaszek, Applied Physics Letters 108, 142101 (2016).

[83] P. Tonndorf, S. Schwarz, J. Kern, I. Niehues, O. Del Pozo-Zamudio, A. I. Dmitriev, A. P. Bakhtinov, D. N. Borisenko, N. N. Kolesnikov, A. I. Tartakovskii, S. Michaelis de Vasconcellos, and R. Bratschitsch, 2D Materials 4, 021010 (2017).

[84] J. Kern, I. Niehues, P. Tonndorf, R. Schmidt, D. Wigger, R. Schneider, T. Stiehm, S. Michaelis de Vasconcellos, D. E. Reiter, T. Kuhn, and R. Bratschitsch, Advanced Materials 28, 7101 (2016).

[85] S. Kumar, A. Kaczmarczyk, and B. D. Gerardot, Nano Letters 15, 7567 (2015), arXiv: 1509.01085.

[86] C. Palacios-Berraquero, D. M. Kara, A. R.-P. Montblanch, M. Barbone, P. Latawiec, D. Yoon, A. K. Ott, M. Loncar, A. C. Ferrari, and M. Atatüre, Nature Communications 8, 15093 (2017).

[87] J. L. O'Brien, A. Furusawa, and J. Vučković, Nature Photonics 3, 687 (2009).

[88] C. Santori, D. Fattal, and Y. Yamamoto, Single-photon devices and applications, Physics textbook (Wiley-VCH, Weinheim, 2010) oCLC: ocn620364663. 
[89] P. Lodahl, S. Mahmoodian, and S. Stobbe, Reviews of Modern Physics 87, $347(2015)$.

[90] F. Xia, H. Wang, D. Xiao, M. Dubey, and A. Ramasubramaniam, Nature Photonics 8, 899 (2014).

[91] A. Branny, S. Kumar, R. Proux, and B. D. Gerardot, Nature Communications 8, 15053 (2017).

[92] M. Born and E. Wolf, Principles of optics: electromagnetic theory of propagation, interference and diffraction of light, 6th ed. (Cambridge University Press, Cambridge, UK, 1997).

[93] S. Tongay, J. Suh, C. Ataca, W. Fan, A. Luce, J. S. Kang, J. Liu, C. Ko, R. Raghunathanan, J. Zhou, F. Ogletree, J. Li, J. C. Grossman, and J. Wu, Scientific Reports 3 (2013), 10.1038/srep02657.

[94] I. Aharonovich, S. Castelletto, D. A. Simpson, A. Stacey, J. McCallum, A. D. Greentree, and S. Prawer, Nano Letters 9, 3191 (2009).

[95] T. Schmidt, K. Lischka, and W. Zulehner, Physical Review B 45, 8989 (1992).

[96] P. YU and M. Cardona, Fundamentals of Semiconductors: Physics and Materials Properties, 3rd ed. (Springer-Verlag, Berlin Heidelberg, 2010).

[97] Y.-M. He, S. Höfling, and C. Schneider, Optics Express 24, 8066 (2016).

[98] Y.-M. He, O. Iff, N. Lundt, V. Baumann, M. Davanco, K. Srinivasan, S. Höfling, and C. Schneider, Nature Communications 7, 13409 (2016).

[99] R. H. Brown and R. Q. Twiss, Nature 177, 27 (1956). 
[100] H. J. Kimble, M. Dagenais, and L. Mandel, Physical Review Letters 39, 691 (1977).

[101] R. Brouri, A. Beveratos, J.-P. Poizat, and P. Grangier, Optics Letters 25, $1294(2000)$.

[102] G. Wang, L. Bouet, D. Lagarde, M. Vidal, A. Balocchi, T. Amand, X. Marie, and B. Urbaszek, Physical Review B 90, 075413 (2014).

[103] A. N. Vamivakas and M. Atatüre, Contemporary Physics 51, 17 (2010).

[104] D. Gammon, E. S. Snow, B. V. Shanabrook, D. S. Katzer, and D. Park, Physical Review Letters 76, 3005 (1996).

[105] M. Bayer, G. Ortner, O. Stern, A. Kuther, A. A. Gorbunov, A. Forchel, P. Hawrylak, S. Fafard, K. Hinzer, T. L. Reinecke, S. N. Walck, J. P. Reithmaier, F. Klopf, and F. Schäfer, Phys. Rev. B 65, 195315 (2002).

[106] Y. Wu, Q. Tong, G.-B. Liu, H. Yu, and W. Yao, Physical Review B 93, 045313 (2016).

[107] D. Akinwande, N. Petrone, and J. Hone, Nature Communications 5, 5678 (2014).

[108] R. Roldán, A. Castellanos-Gomez, E. Cappelluti, and F. Guinea, Journal of Physics: Condensed Matter 27, 313201 (2015).

[109] S. Manzeli, A. Allain, A. Ghadimi, and A. Kis, Nano Letters 15, 5330 (2015).

[110] Y. Y. Hui, X. Liu, W. Jie, N. Y. Chan, J. Hao, Y.-T. Hsu, L.-J. Li, W. Guo, and S. P. Lau, ACS Nano 7, 7126 (2013). 
[111] A. Kormányos, V. Zólyomi, N. D. Drummond, and G. Burkard, Physical Review X 4 (2014), 10.1103/PhysRevX.4.011034.

[112] C. Chakraborty, K. M. Goodfellow, S. Dhara, A. Yoshimura, V. Meunier, and N. Vamivakas, Nano Letters 17, 2253 (2017).

[113] H. Fang, C. Battaglia, C. Carraro, S. Nemsak, B. Ozdol, J. S. Kang, H. A. Bechtel, S. B. Desai, F. Kronast, A. A. Unal, G. Conti, C. Conlon, G. K. Palsson, M. C. Martin, A. M. Minor, C. S. Fadley, E. Yablonovitch, R. Maboudian, and A. Javey, Proceedings of the National Academy of Sciences 111, 6198 (2014).

[114] R. J. Warburton, C. Schaflein, D. Haft, F. Bickel, A. Lorke, K. Karrai, J. M. Garcia, W. Schoenfeld, and P. M. Petroff, Nature 405, 926 (2000).

[115] T. Georgiou, R. Jalil, B. D. Belle, L. Britnell, R. V. Gorbachev, S. V. Morozov, Y.-J. Kim, A. Gholinia, S. J. Haigh, O. Makarovsky, L. Eaves, L. A. Ponomarenko, A. K. Geim, K. S. Novoselov, and A. Mishchenko, Nature Nanotechnology 8, 100 (2012).

[116] F. Federspiel, G. Froehlicher, M. Nasilowski, S. Pedetti, A. Mahmood, B. Doudin, S. Park, J.-O. Lee, D. Halley, B. Dubertret, P. Gilliot, and S. Berciaud, Nano Letters 15, 1252 (2015).

[117] Y.-H. Kuo, Y. K. Lee, Y. Ge, S. Ren, J. E. Roth, T. I. Kamins, D. A. Miller, and J. S. Harris, Nature 437, 1334 (2005).

[118] K. Park, Z. Deutsch, J. J. Li, D. Oron, and S. Weiss, ACS Nano 6, 10013 (2012). 
[119] P. Tamarat, T. Gaebel, J. R. Rabeau, M. Khan, A. D. Greentree, H. Wilson, L. C. L. Hollenberg, S. Prawer, P. Hemmer, F. Jelezko, and J. Wrachtrup, Phys. Rev. Lett. 97, 083002 (2006).

[120] K. K. Kim, A. Hsu, X. Jia, S. M. Kim, Y. Shi, M. Dresselhaus, T. Palacios, and J. Kong, ACS Nano 6, 8583 (2012).

[121] A. J. Bennett, R. B. Patel, J. Skiba-Szymanska, C. A. Nicoll, I. Farrer, D. A. Ritchie, and A. J. Shields, Applied Physics Letters 97, 031104 (2010).

[122] T. Müller, I. Aharonovich, L. Lombez, Y. Alaverdyan, A. N. Vamivakas, S. Castelletto, F. Jelezko, J. Wrachtrup, S. Prawer, and M. Atatüre, New Journal of Physics 13, 075001 (2011).

[123] R. J. Warburton, C. Schulhauser, D. Haft, C. Schäflein, K. Karrai, J. M. Garcia, W. Schoenfeld, and P. M. Petroff, Physical Review B 65 (2002), 10.1103/PhysRevB.65.113303.

[124] S. Cao, J. Tang, Y. Gao, Y. Sun, K. Qiu, Y. Zhao, M. He, J.-A. Shi, L. Gu, D. A. Williams, W. Sheng, K. Jin, and X. Xu, Scientific Reports 5, 8041 (2015).

[125] J. Beugnon, M. P. A. Jones, J. Dingjan, B. Darquié, G. Messin, A. Browaeys, and P. Grangier, Nature 440, 779 (2006).

[126] E. A. Stinaff, M. Scheibner, A. S. Bracker, I. V. Ponomarev, V. L. Korenev, M. E. Ware, M. F. Doty, T. L. Reinecke, and D. Gammon, Science 311, $636(2006)$.

[127] M. T. Rakher, N. G. Stoltz, L. A. Coldren, P. M. Petroff, and D. Bouwmeester, Physical Review Letters 102, 097403 (2009). 
[128] A. J. Bennett, M. A. Pooley, R. M. Stevenson, M. B. Ward, R. B. Patel, A. B. de la Giroday, N. Sköld, I. Farrer, C. A. Nicoll, D. A. Ritchie, and A. J. Shields, Nature Physics 6, 947 (2010).

[129] H. J. Kimble, Nature 453, 1023 (2008).

[130] S. Kumar, E. Zallo, Y. H. Liao, P. Y. Lin, R. Trotta, P. Atkinson, J. D. Plumhof, F. Ding, B. D. Gerardot, S. J. Cheng, A. Rastelli, and O. G. Schmidt, Physical Review B 89 (2014), 10.1103/PhysRevB.89.115309.

[131] R. Trotta, P. Atkinson, J. D. Plumhof, E. Zallo, R. O. Rezaev, S. Kumar, S. Baunack, J. R. Schröter, A. Rastelli, and O. G. Schmidt, Advanced Materials 24, 2668 (2012).

[132] M. Z. Maialle, E. A. de Andrada e Silva, and L. J. Sham, Physical Review B 47, 15776 (1993).

[133] R. Trotta, E. Zallo, C. Ortix, P. Atkinson, J. D. Plumhof, J. van den Brink, A. Rastelli, and O. G. Schmidt, Physical Review Letters 109, 147401 (2012).

[134] M. Ghali, Y. Ohno, and H. Ohno, Applied Physics Letters 107, 123102 (2015).

[135] C. Böckler, S. Reitzenstein, C. Kistner, R. Debusmann, A. Löffler, T. Kida, S. Höfling, A. Forchel, L. Grenouillet, J. Claudon, and J. M. Gérard, Applied Physics Letters 92, 091107 (2008).

[136] C. Chakraborty, L. Qiu, K. Konthasinghe, A. Mukherjee, S. Dhara, and N. Vamivakas, Nano Letters (2018), 10.1021/acs.nanolett.7b05409.

[137] M. I. Dyakonov, ed., Spin physics in semiconductors, Springer series in solidstate sciences No. 157 (Springer, Berlin, 2008) oCLC: ocn222164414. 
[138] I. Žutić, J. Fabian, and S. Das Sarma, Reviews of Modern Physics 76, 323 (2004).

[139] A. İmamoğlu, D. D. Awschalom, G. Burkard, D. P. DiVincenzo, D. Loss, M. Sherwin, and A. Small, Physical Review Letters 83, 4204 (1999).

[140] R. Hanson and D. D. Awschalom, Nature 453, 1043 (2008).

[141] C. Bonato, M. S. Blok, H. T. Dinani, D. W. Berry, M. L. Markham, D. J. Twitchen, and R. Hanson, Nature Nanotechnology 11, 247 (2016).

[142] I. Jakobi, P. Neumann, Y. Wang, D. B. R. Dasari, F. El Hallak, M. A. Bashir, M. Markham, A. Edmonds, D. Twitchen, and J. Wrachtrup, Nature Nanotechnology 12, 67 (2016).

[143] A. N. Vamivakas, C.-Y. Lu, C. Matthiesen, Y. Zhao, S. Fält, A. Badolato, and M. Atatüre, Nature 467, 297 (2010).

[144] S. Kumar, M. Brotóns-Gisbert, R. Al-Khuzheyri, G. Ballesteros-Garcia, J. F. Sánchez-Royo, and B. D. Gerardot, Optica 3, 882 (2016).

[145] H. Drexler, D. Leonard, W. Hansen, J. P. Kotthaus, and P. M. Petroff, Physical Review Letters 73, 2252 (1994).

[146] M. Zieliński, K. Gołasa, M. R. Molas, M. Goryca, T. Kazimierczuk, T. Smoleński, A. Golnik, P. Kossacki, A. A. L. Nicolet, M. Potemski, Z. R. Wasilewski, and A. Babiński, Physical Review B 91 (2015), 10.1103/PhysRevB.91.085303.

[147] S. Seidl, M. Kroner, P. A. Dalgarno, A. Högele, J. M. Smith, M. Ediger, B. D. Gerardot, J. M. Garcia, P. M. Petroff, K. Karrai, and R. J. Warburton, Physical Review B 72, 195339 (2005). 
[148] G. Aivazian, Z. Gong, A. M. Jones, R.-L. Chu, J. Yan, D. G. Mandrus, C. Zhang, D. Cobden, W. Yao, and X. Xu, Nature Physics 11, 148 (2015).

[149] J. Tang, S. Cao, Y. Gao, Y. Sun, W. Geng, D. A. Williams, K. Jin, and X. Xu, Appl. Phys. Lett. 105, (2014).

[150] A. S. Bracker, E. A. Stinaff, D. Gammon, M. E. Ware, J. G. Tischler, A. Shabaev, A. L. Efros, D. Park, D. Gershoni, V. L. Korenev, and I. A. Merkulov, Physical Review Letters 94, 047402 (2005).

[151] G. Plechinger, P. Nagler, A. Arora, R. Schmidt, A. Chernikov, A. G. d. Águila, P. C. M. Christianen, R. Bratschitsch, C. Schüller, and T. Korn, Nature Communications 7, 12715 (2016).

[152] E. Courtade, M. Semina, M. Manca, M. M. Glazov, C. Robert, F. Cadiz, G. Wang, T. Taniguchi, K. Watanabe, M. Pierre, W. Escoffier, E. L. Ivchenko, P. Renucci, X. Marie, T. Amand, and B. Urbaszek, Physical Review B 96 (2017), 10.1103/PhysRevB.96.085302.

[153] D. Press, T. D. Ladd, B. Zhang, and Y. Yamamoto, Nature 456, 218 (2008).

[154] J. Kim, X. Hong, C. Jin, S.-F. Shi, C.-Y. S. Chang, M.-H. Chiu, L.-J. Li, and F. Wang, Science 346, 1205 (2014).

[155] C. Chakraborty, R. Beams, K. M. Goodfellow, G. W. Wicks, L. Novotny, and A. N. Vamivakas, Applied Physics Letters 105, 241114 (2014).

[156] K. S. Novoselov, A. K. Geim, S. V. Morozov, D. Jiang, Y. Zhang, S. V. Dubonos, I. V. Grigorieva, and A. A. Firsov, Science 306, 666 (2004).

[157] R. R. Nair, P. Blake, A. N. Grigorenko, K. S. Novoselov, T. J. Booth, T. Stauber, N. M. R. Peres, and A. K. Geim, Science 320, 1308 (2008). 
[158] C. Lee, X. Wei, J. W. Kysar, and J. Hone, Science 321, 385 (2008).

[159] K. I. Bolotin, K. J. Sikes, Z. Jiang, M. Klima, G. Fudenberg, J. Hone, P. Kim, and H. L. Stormer, Solid State Communications 146, 351 (2008).

[160] F. Bonaccorso, Z. Sun, T. Hasan, and A. C. Ferrari, Nature Photonics 4, $611(2010)$.

[161] J. B. Khurgin and G. Sun, Journal of the Optical Society of America B 26, B83 (2009).

[162] V. M. Shalaev, C. Douketis, J. T. Stuckless, and M. Moskovits, Phys. Rev. B 53, 11388 (1996).

[163] L. Novotny and B. Hecht, Principles of Nano-Optics (Cambridge University Press, Cambridge, 2006).

[164] A. G. Curto, G. Volpe, T. H. Taminiau, M. P. Kreuzer, R. Quidant, and N. F. van Hulst, Science 329, 930 (2010).

[165] O. L. Muskens, V. Giannini, J. A. Sánchez-Gil, and J. Gómez Rivas, Nano Letters 7, 2871 (2007).

[166] Y. Shi, J.-K. Huang, L. Jin, Y.-T. Hsu, S. F. Yu, L.-J. Li, and H. Y. Yang, Scientific Reports 3 (2013), 10.1038/srep01839.

[167] J. Niu, Y. Jun Shin, Y. Lee, J.-H. Ahn, and H. Yang, Applied Physics Letters 100, 061116 (2012).

[168] Z. Fang, Z. Liu, Y. Wang, P. M. Ajayan, P. Nordlander, and N. J. Halas, Nano Letters 12, 3808 (2012). 
[169] Y. Yao, M. A. Kats, P. Genevet, N. Yu, Y. Song, J. Kong, and F. Capasso, Nano Letters 13, 1257 (2013).

[170] T. J. Echtermeyer, L. Britnell, P. K. Jasnos, A. Lombardo, R. V. Gorbachev, A. N. Grigorenko, A. K. Geim, A. C. Ferrari, and K. S. Novoselov, Nature Communications 2, 458 (2011).

[171] N. K. Emani, T.-F. Chung, X. Ni, A. V. Kildishev, Y. P. Chen, and A. Boltasseva, Nano Letters 12, 5202 (2012).

[172] Y. Yao, R. Shankar, P. Rauter, Y. Song, J. Kong, M. Loncar, and F. Capasso, Nano Letters 14, 3749 (2014).

[173] L. Vicarelli, M. S. Vitiello, D. Coquillat, A. Lombardo, A. C. Ferrari, W. Knap, M. Polini, V. Pellegrini, and A. Tredicucci, Nature Materials 11, 865 (2012).

[174] M. Mittendorff, S. Winnerl, J. Kamann, J. Eroms, D. Weiss, H. Schneider, and M. Helm, Applied Physics Letters 103, 021113 (2013).

[175] S.-F. Shi, X. Xu, D. C. Ralph, and P. L. McEuen, Nano Letters 11, 1814 (2011).

[176] M. Dvorak, W. Oswald, and Z. Wu, Scientific Reports 3 (2013), 10.1038/srep02289.

[177] L. Genzel, T. P. Martin, and U. Kreibig, Zeitschrift für Physik B Condensed Matter 21, 339 (1975).

[178] Z. Liu, A. Boltasseva, R. H. Pedersen, R. Bakker, A. V. Kildishev, V. P. Drachev, and V. M. Shalaev, Metamaterials 2, 45 (2008). 
[179] A. L. Falk, F. H. L. Koppens, C. L. Yu, K. Kang, N. d. L. Snapp, A. V. Akimov, M.-H. Jo, M. D. Lukin, and H. Park, Nature Phys 5, 475 (2009).

[180] J. Park, Y. H. Ahn, and C. Ruiz-Vargas, Nano Letters 9, 1742 (2009).

[181] E. J. H. Lee, K. Balasubramanian, R. T. Weitz, M. Burghard, and K. Kern, Nature Nanotechnology 3, 486 (2008).

[182] F. Xia, T. Mueller, Y.-m. Lin, A. Valdes-Garcia, and P. Avouris, Nature Nanotechnology 4, 839 (2009).

[183] X. Xu, N. M. Gabor, J. S. Alden, A. M. van der Zande, and P. L. McEuen, Nano Letters 10, 562 (2010).

[184] K. M. Goodfellow, C. Chakraborty, R. Beams, L. Novotny, and A. N. Vamivakas, Nano Letters 15, 5477 (2015).

[185] R. F. Oulton, V. J. Sorger, D. A. Genov, D. F. P. Pile, and X. Zhang, Nature Photonics 2, 496 (2008).

[186] J. Rattner, in Integrated Photonics Research, Silicon and Nanophotonics and Photonics in Switching (Optical Society of America, 2010) p. Paper JTuA1.

[187] D. Pacifici, H. J. Lezec, and H. A. Atwater, Nature Photonics 1, 402 (2007).

[188] S. A. Maier and H. A. Atwater, J. Appl. Phys. 98, 011101 (2005).

[189] W. L. Barnes, A. Dereux, and T. W. Ebbeseni, Nature 424, 824 (2003).

[190] K. C. Y. Huang, M.-K. Seo, T. Sarmiento, Y. Huo, J. S. Harris, and M. L. Brongersma, Nature Photon. 8, 244 (2014).

[191] M. Liu, X. Yin, E. Ulin-Avila, B. Geng, T. Zentgraf, L. Ju, F. Wang, and X. Zhang, Nature 474, 64 (2011). 
[192] S. Assefa, F. Xia, and Y. A. Vlasov, Nature 464, 80 (2010).

[193] H. Ditlbacher, A. Hohenau, D. Wagner, U. Kreibig, M. Rogers, F. Hofer, F. R. Aussenegg, and J. R. Krenn, Phys. Rev. Lett. 95, 257403 (2005).

[194] Z. Li, K. Bao, Y. Fang, Y. Huang, P. Nordlander, and H. Xu, Nano Letters 10, 1831 (2010).

[195] A. W. Sanders, D. A. Routenberg, B. J. Wiley, Y. Xia, E. R. Dufresne, and M. A. Reed, Nano Letters 6, 1822 (2006).

[196] A. V. Akimov, A. Mukherjee, C. L. Yu, D. E. Chang, A. S. Zibrov, P. R. Hemmer, H. Park, and M. D. Lukin, Nature 450, 402 (2007).

[197] K. M. Goodfellow, R. Beams, C. Chakraborty, L. Novotny, and A. N. Vamivakas, Optica 1, 149 (2014).

[198] H. Wei, D. Ratchford, X. Li, H. Xu, and C.-K. Shih, Nano Lett. 9, 4168 (2009).

[199] A. Huck, S. Kumar, A. Shakoor, and U. L. Andersen, Phys. Rev. Lett. 106, 096801 (2011).

[200] H. Qian, Y. Ma, Q. Yang, B. Chen, Y. Liu, X. Guo, S. Lin, J. Ruan, X. Liu, L. Tong, and Z. L. Wang, ACS Nano 8, 2584 (2014).

[201] O. Lopez-Sanchez, D. Lembke, M. Kayci, A. Radenovic, and A. Kis, Nature Nanotechnology 8, 497 (2013).

[202] Z. Yin, H. Li, H. Li, L. Jiang, Y. Shi, Y. Sun, G. Lu, Q. Zhang, X. Chen, and H. Zhang, ACS Nano 6, 74 (2012). 
[203] C.-C. Wu, D. Jariwala, V. K. Sangwan, T. J. Marks, M. C. Hersam, and L. J. Lauhon, The Journal of Physical Chemistry Letters 4, 2508 (2013).

[204] S. Dhara, C. Chakraborty, K. M. Goodfellow, L. Qiu, T. A. O’Loughlin, G. W. Wicks, S. Bhattacharjee, and A. N. Vamivakas, Nature Physics 14, $130(2018)$.

[205] R. Balili, V. Hartwell, D. Snoke, L. Pfeiffer, and K. West, Science 316, 1007 (2007).

[206] H. Deng, G. Weihs, D. Snoke, J. Bloch, and Y. Yamamoto, Proceedings of the National Academy of Sciences 100, 15318 (2003).

[207] S. I. Tsintzos, N. T. Pelekanos, G. Konstantinidis, Z. Hatzopoulos, and P. G. Savvidis, Nature 453, 372 (2008).

[208] C. Weisbuch, M. Nishioka, A. Ishikawa, and Y. Arakawa, Physical Review Letters 69, 3314 (1992).

[209] A. Kavokin, physica status solidi (b) 247, 1898 (2010).

[210] X. Liu, T. Galfsky, Z. Sun, F. Xia, E.-c. Lin, Y.-H. Lee, S. Kéna-Cohen, and V. M. Menon, Nature Photonics 9, 30 (2015).

[211] M. Sidler, P. Back, O. Cotlet, A. Srivastava, T. Fink, M. Kroner, E. Demler, and A. Imamoglu, Nature Physics 13, 255 (2016).

[212] S. Dufferwiel, S. Schwarz, F. Withers, A. A. P. Trichet, F. Li, M. Sich, O. Del Pozo-Zamudio, C. Clark, A. Nalitov, D. D. Solnyshkov, G. Malpuech, K. S. Novoselov, J. M. Smith, M. S. Skolnick, D. N. Krizhanovskii, and A. I. Tartakovskii, Nature Communications 6, 8579 (2015). 
[213] J. S. Ross, S. Wu, H. Yu, N. J. Ghimire, A. M. Jones, G. Aivazian, J. Yan, D. G. Mandrus, D. Xiao, W. Yao, and X. Xu, Nature Communications 4, 1474 (2013).

[214] M. Wurdack, N. Lundt, M. Klaas, V. Baumann, A. V. Kavokin, S. Höfling, and C. Schneider, Nature Communications 8, 259 (2017).

[215] X. Liu, T. Galfsky, Z. Sun, F. Xia, E.-c. Lin, Y.-H. Lee, S. Kéna-Cohen, and V. M. Menon, arXiv:1406.4826 [cond-mat, physics:physics] (2014), arXiv: 1406.4826.

[216] G. D. Shepard, O. A. Ajayi, X. Li, X.-Y. Zhu, J. Hone, and S. Strauf, 2D Materials 4, 021019 (2017).

[217] M. D. Fraser, Semiconductor Science and Technology 32, 093003 (2017).

[218] J. Rudolph, D. Hägele, H. M. Gibbs, G. Khitrova, and M. Oestreich, Applied Physics Letters 82, 4516 (2003).

[219] K. F. Mak and J. Shan, Nature Photonics 10, 216 (2016). 